\title{
MICHEL HICKEL
}

\section{Sur quelques aspects de la géométrie de l'espace des arcs tracés sur un espace analytique}

\author{
Annales de la faculté des sciences de Toulouse $\sigma^{e}$ série, tome 14, \\ $\mathrm{n}^{\mathrm{o}} 1$ (2005), p. 1-50 \\ <http://www.numdam.org/item?id=AFST_2005_6_14_1_1_0>
}

(C) Université Paul Sabatier, 2005, tous droits réservés.

L'accès aux archives de la revue «Annales de la faculté des sciences de Toulouse » (http://picard.ups-tlse.fr/ annales/) implique l'accord avec les conditions générales d'utilisation (http://www.numdam.org/conditions). Toute utilisation commerciale ou impression systématique est constitutive d'une infraction pénale. Toute copie ou impression de ce fichier doit contenir la présente mention de copyright.

\section{NumDam}

Article numérisé dans le cadre du programme

Numérisation de documents anciens mathématiques

http://www.numdam.org/ 


\title{
Sur quelques aspects de la géométrie de l'espace des arcs tracés sur un espace analytique ${ }^{(*)}$
}

\author{
MICHEL HICKEL ${ }^{(1)}$
}

\begin{abstract}
RÉSUMÉ. - Soient $(X, x)$ un germe d'espace analytique complexe ou réel et $\mathcal{A}_{(X, x)}$ l'espace des arcs tracés sur $(X, x)$. Considérons $F_{x}:(X, x) \rightarrow$ $(Y, y)$ un germe de morphisme et désignons par $\mathcal{F}_{x}: \mathcal{A}_{(X, x)} \rightarrow \mathcal{A}_{(Y, y)}$ le morphisme induit au niveau des espaces des arcs. Dans cet article, nous tentons de souligner les analogies entre les propriétés topologiques locales ou métriques de $F_{x}$ et celles de $\mathcal{F}_{x}$. Nous définissons ensuite les notions de suite de Nash des multiplicités, suite de Nash des fonctions de Hilbert-Samuel, suite de Nash des diagrammes des exposants initiaux de $X$ le long d'un $\operatorname{arc} \varphi$, et étudions leurs premières propriétés. Certains liens élémentaires avec la théorie de l'integration motivique sont aussi établis.
\end{abstract}

\begin{abstract}
Let $(X, x)$ be a germ of real or complex analytic space and $\mathcal{A}_{(X, x)}$ the space of germs of arcs on $(X, x)$. Let us consider $F_{x}$ : $(X, x) \rightarrow(Y, y)$ a germ of a morphism and denote by $\mathcal{F}_{x}: \mathcal{A}_{(X, x)} \rightarrow$ $\mathcal{A}_{(Y, y)}$ the induced morphism at the level of arcs. In this paper, we try to emphasize the analogies between the metric or local topological properties of $F_{x}$ and those of $\mathcal{F}_{x}$. We then define the notions of Nash sequence of multiplicities, Nash sequence of Hilbert-Samuel functions and Nash sequence of diagram of initial exponents of $X$ along $\operatorname{an} \operatorname{arc} \varphi$, and study some of their basic properties. Some elementary connections between these notions and motivic integration theory are also provided.
\end{abstract}

\section{Introduction}

Soient $\mathbb{K}=\mathbb{R}$ ou $\mathbb{C}$ et $(X, x)$ un germe de $\mathbb{K}$-espace analytique, d'algèbre locale $\mathcal{O}_{X, x}$. Nous noterons par $\mathcal{A}_{(X, x)}$ l'espace des arcs tracés sur $(X, x)$, c'est-à-dire l'ensemble des germes de morphismes analytiques $\varphi$ de $(\mathbb{K}, 0)$ dans $(X, x)$, ou bien de façon équivalente l'ensemble des morphismes $\varphi^{*}$ de

(*) Reçu le 26 février 2003, accepté le 25 mai 2004

(1) Université Bordeaux 1, Laboratoire d'analyse et géométrie, U.M.R. $5467 \mathrm{du}$ C.N.R.S., 351 cours de la libération, F-33405 TALENCE Cedex.

E-mail : hickel@math.u-bordeaux.fr 
$\mathbb{K}$-algèbre locale de $\mathcal{O}_{X, x}$ dans $\mathcal{O}_{1}=\mathbb{K}\{t\}$. De manière plus générale si $X$ est un espace analytique et $A$ un sous-ensemble de $|X|$, nous désignerons par $\mathcal{A}_{(X, A)}$ (resp. $\mathcal{A}_{X}$ ) l'union $\coprod_{x \in A} \mathcal{A}_{(X, x)}$ (resp. $\left.\coprod_{x \in X} \mathcal{A}_{(X, x)}\right)$. $\mathcal{A}_{X}$ est naturellement munie d'une distance ultramétrique notée $\mathcal{D}_{X}$, définie de la manière suivante :

- $\forall \varphi \in \mathcal{A}_{(X, x)}, \forall \phi \in \mathcal{A}_{\left(X, x^{\prime}\right)}, x \neq x^{\prime}$, on pose $\mathcal{D}_{X}(\varphi, \phi)=1 ;$

- $\forall \varphi, \phi \in \mathcal{A}_{(X, x)}$, on pose $\mathcal{D}_{X}(\varphi, \phi)=e^{-\operatorname{ord}(\varphi-\phi)}$, où $\operatorname{ord}(\varphi-\phi)$ désigne la valuation ou l'ordre de l'idéal de $\mathcal{O}_{1},\left(\varphi^{*}-\phi^{*}\right)\left(\mathcal{M}_{X, x}\right)$, et $\mathcal{M}_{X, x}$ l'idéal maximal de $\mathcal{O}_{X, x}$.

La topologie induite par $\mathcal{D}_{X}$ sur $\mathcal{A}_{X}$ sera dite la topologie de Krull sur $\mathcal{A}_{X}$. Soit maintenant un germe de morphisme analytique $F_{x}:(X, x) \rightarrow$ $(Y, y)$. Celui-ci induit un morphisme $\mathcal{F}_{x}: \mathcal{A}_{(X, x)} \rightarrow \mathcal{A}_{(Y, y)}$, où pour $\varphi \in$ $\mathcal{A}_{(X, x)}, \mathcal{F}_{x}(\varphi)$ est l'arc $(\mathbb{K}, 0) \rightarrow(X, x) \rightarrow(Y, y)$ obtenu par composition de $\varphi$ avec $F_{x}$. De façon plus générale si $F: X \rightarrow Y$ est un morphisme, nous noterons par $\mathcal{F}: \mathcal{A}_{X} \rightarrow \mathcal{A}_{Y}$ le morphisme induit au niveau de l'espace des arcs.

Dans cet article nous abordons deux thèmes. Le premier consiste à tenter de souligner les analogies entre les propriétés topologiques locales d'un morphisme $F: X \rightarrow Y$, et celles du morphisme induit $\mathcal{F}: \mathcal{A}_{X} \rightarrow \mathcal{A}_{Y}$.

Nous donnons deux illustrations de ce jeu de miroirs. La première concerne un résultat du à J.C. Tougeron. Dans [T3], l'auteur précité donne des inégalités de Lojasiewicz, avec exposant uniforme, par rapport aux fibres d'un morphisme analytique. Plus précisément :

ThÉORÈme 1.1 (J.C. TOUgeron ). - Soit $F$ une application analytique d'un ouvert $\Omega_{n}$ de $\mathbb{R}^{n}$ dans un ouvert $\Omega_{p}$ de $\mathbb{R}^{p}$. Pour toute paire de compacts $K \subset \Omega_{n}$ et $K^{\prime} \subset \Omega_{p}$, il existe une constante $\alpha \geqslant 0$ satisfaisant :

$$
\forall y \in K^{\prime}, \exists C_{y}>0 \mid \forall x \in K, d_{p}(F(x), y) \geqslant C_{y} d_{n}\left(x, F^{-1}(y)\right)^{\alpha}
$$

où $d_{p}$ (resp. $d_{n}$ ) désigne la distance euclidienne sur $\mathbb{R}^{p}$ (resp. sur $\mathbb{R}^{n}$ ).

Le théorème résulte trivialement de l'assertion suivante :

Assertion 1.2. - Pour toute paire de compacts $K \subset \Omega_{n}, K^{\prime} \subset \Omega_{p}$, il existe une constante $\alpha \geqslant 0$ satisfaisant :

$\forall y \in K^{\prime}, \forall x_{0} \in K, \exists C_{\left(x_{0}, y\right)}>0$ et $V_{x_{0}}$ voisinage de $x_{0}$ tel que :

$$
\forall x \in V_{x_{0}}, d_{p}(F(x), y) \geqslant C_{\left(x_{0}, y\right)} d_{n}\left(x, F^{-1}(y)\right)^{\alpha}
$$


Nous allons voir que l'on a un résultat analogue, au niveau du morphisme induit au niveau des espaces des arcs, en remplaçant la distance euclidienne par la distance ultramétrique définie plus haut. Nous démontrerons en effet :

ThÉORÈme 1.3. - Soit $F: X \rightarrow Y$ un morphisme de $\mathbb{K}$-espace analytique et $\mathcal{F}: \mathcal{A}_{X} \rightarrow \mathcal{A}_{Y}$ le morphisme induit au niveau de l'espace des arcs. Alors pour toute paire de compacts $K \subset X, K^{\prime} \subset Y$, il existe une constante $\alpha$ satisfaisant :

$\forall \varphi \in \mathcal{A}_{\left(Y, K^{\prime}\right)}, \forall \phi_{0} \in \mathcal{A}_{(X, K)}, \exists C_{\left(\phi_{0}, \varphi\right)}>0$ et $\mathcal{V} \phi_{0}$ voisinage de $\phi_{0}$ tel que :

$$
\forall \phi \in \mathcal{V}_{\phi_{0}}, \mathcal{D}_{X}(\mathcal{F}(\phi), \varphi) \geqslant C_{\left(\phi_{0}, \varphi\right)} \mathcal{D}_{Y}\left(\phi, \mathcal{F}^{-1}(\varphi)\right)^{\alpha} .
$$

De la même façon que l'existence d'une majoration affine pour la fonction d'Artin-Greenberg (cf. [Gr]) équivaut à des inégalités de Lojasiewicz au niveau de l'espace des arcs (cf. [H2]), le résultat ci-dessus s'interprète comme une version partiellement uniforme du théorème de Greenberg, l'espace des paramètres étant $\mathcal{A}_{Y}$ (cf. section 3 ).

La seconde illustration des analogies précédemment évoquées concerne la condition de rang de A.M. Gabrielov. Pour le rôle joué par cette condition, nous renvoyons le lecteur à [B-M3], [Ga], [I1], [I2], [I3], [I4], [P] et plus particulièrement à [I4] et à sa bibliographie pour une historique. Si $F_{x}:(X, x) \rightarrow(Y, y)$ est un germe de morphisme entre germes d'espaces irréductibles, on définit le rang générique de $F_{x}$ par :

$$
\operatorname{grk}\left(F_{x}\right)=\inf _{U}\left(\sup _{x^{\prime}} \operatorname{rang}\left(F_{x^{\prime}}\right), x^{\prime} \in \operatorname{Reg}(X \cap U)\right)
$$

où $U$ parcourt une base de voisinage de $x, \operatorname{Reg}(X \cap U)$ désigne l'ensemble des points réguliers de $X \cap U$, et $\operatorname{rang}\left(F_{x^{\prime}}\right)$ est le rang de la matrice jacobienne de $F_{x^{\prime}}$ en $x^{\prime}$. On dit que $F_{x}$ satisfait la condition de rang de A.M. Gabrielov si et seulement si :

$$
\operatorname{grk}\left(F_{x}\right)=\operatorname{dim}_{y} Y .
$$

Pour $\mathbb{K}=\mathbb{C}$, il est relativement élémentaire de vérifier que cette condition peut se caractériser topologiquement par l'équivalence des conditions suivantes :

$-\operatorname{grk}\left(F_{x}\right)=\operatorname{dim}_{y} Y$

- $\forall U$ voisinage de $x$ assez petit, $\forall V$ ouvert de $\operatorname{Reg}(X \cap U), F_{x}(V)$ contient un ouvert de $\operatorname{Reg}(Y)$;

- $\forall U$ voisinage de $x$ assez petit, $F_{x}(\operatorname{Reg}(X \cap U))$ contient un ouvert de $\operatorname{Reg}(Y)$. 
Désignons maintenant par $(\operatorname{Sing}(X), x)$ le germe en $x$ de l'ensemble des points singuliers de $(X, x)$. Soit alors $\mathcal{R}_{(X, x)}=\mathcal{A}_{(X, x)}-\mathcal{A}_{(\operatorname{sing}(X), x)}$ l'ensemble des arcs tracés dans $(X, x)$, mais dont l'image n'est pas entièrement incluse dans $(\operatorname{Sing}(X), x)$. $\mathcal{R}_{(X, x)}$ joue comme nous le verrons plus loin le rôle de l'ensemble des points réguliers de $\mathcal{A}_{(X, x)}$. Etant donné un morphisme $F_{x}:(X, x) \rightarrow(Y, y)$, il semble naturel de se demander quand $\mathcal{F}_{x}\left(\mathcal{A}_{(X, x)}\right)$ contient un ensemble représentatif de $\mathcal{A}_{(Y, y)}$, par exemple un ouvert de $\mathcal{R}_{(Y, y)}$. Là encore le phénomène est similaire au niveau du morphisme $F_{x}$ et à celui de $\mathcal{F}_{x}$. On a en effet :

ThÉORÈme 1.4. - Pour $\mathbb{K}=\mathbb{C}$, soient $F_{x}:(X, x) \rightarrow(Y, y)$ un morphisme entre deux germes irréductibles et $\mathcal{F}_{x}: \mathcal{A}_{(X, x)} \rightarrow \mathcal{A}_{(Y, y)}$ le morphisme induit. Les propriétés suivantes sont équivalentes :

1) $\operatorname{grk}\left(F_{x}\right)=\operatorname{dim}_{y} Y$;

2) Pour tout ouvert $\mathcal{V}$ de $\mathcal{R}_{(X, x)}, \mathcal{F}_{x}(\mathcal{V})$ contient un ouvert de $\mathcal{R}_{(Y, y)}$;

3) $\mathcal{F}_{x}\left(\mathcal{R}_{(X, x)}\right)$ contient un ouvert de $\mathcal{R}_{(Y, y)}$.

Notre démonstration est élémentaire dans le sens où elle n'utilise pas l'existence de désingularisation, ni aucun des résultats concernant la condition de rang de A.M. Gabrielov mentionnés ci-dessus. Par contre on peut retrouver partiellement à partir de 1.4 certains de ces résultats (cf. section 6).

Le deuxième thème abordé dans cet article concerne la notion de suite des multiplicités de Nash le long d'un arc. En un certain sens, nous cherchons des notions convenables de multiplicité, de fonction de Hilbert-Samuel, et de diagramme des exposants initiaux de $\mathcal{A}_{(X, x)}$ en un point $\varphi$. Dans [L-J], M. Lejeune-Jalabert introduisait de manière algorithmique, pour un germe d'hypersurface $(H, x)$, la notion de suite des multiplicités de Nash de $(H, x)$ le long d'un arc. Dans notre travail [H1] également consacré aux hypersurfaces, cette notion apparaissait sous un point de vue plus géométrique. Nous généralisons ici ce point de vue, pour définir les notions de suite des multiplicités de Nash, suite de Nash des fonctions de Hilbert-Samuel et suite de Nash des diagrammes des exposants initiaux de $(X, x)$ le long d'un $\operatorname{arc} \varphi$, pour tout germe $(X, x)$.

Les définitions sont les suivantes. Considérons un plongement $i_{0}$ de $(X, x)$ dans un $\left(\mathbb{K}^{n}, 0\right)$. Ceci induit un plongement de $\mathcal{A}_{(X, x)}$ dans $\mathcal{A}_{\left(\mathbb{K}^{n}, 0\right)}$. Soit $\varphi \in \mathcal{A}_{\left(\mathbb{K}^{n}, 0\right)}$. On pose : 
$-\left(E_{0}, O_{0}\right)=(\mathbb{K}, 0) \times\left(\mathbb{K}^{n}, 0\right)=\left(\mathbb{K}^{n+1}, 0\right) ;$

- $\left(Z_{0}, O_{0}\right)=(\mathbb{K}, 0) \times(X, x) ;$

- $\Gamma_{0}:(\mathbb{K}, 0) \rightarrow\left(E_{0}, O_{0}\right)$ le graphe de $\varphi$.

On considère alors le diagramme commutatif $\left(D_{1}\right)$ suivant :

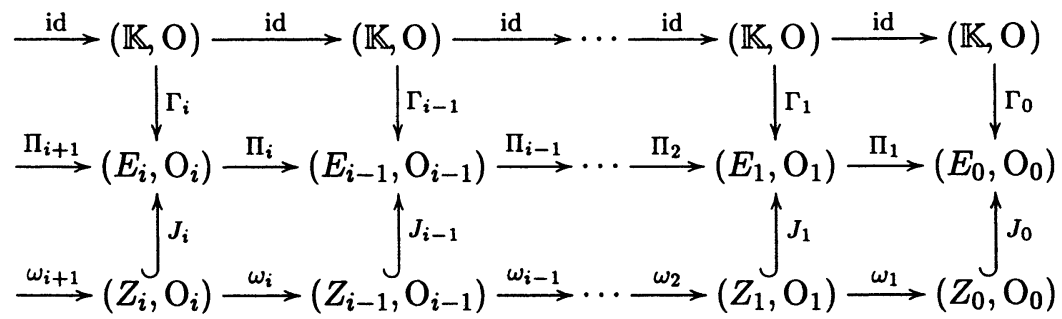

où pour tout $i \geqslant 1$ :

- $\Pi_{i}$ est l'éclatement de $E_{i-1}$ de centre $O_{i-1}$;

- $\Gamma_{i}$ est le relèvement de $\Gamma_{i-1}$ à travers $\Pi_{i}$ et $O_{i}=\Gamma_{i}(0)$;

- $Z_{i}$ est le transformé strict de $Z_{i-1}$ par $\Pi_{i}$ et $\omega_{i}=\Pi_{i} \mid Z_{i}, J_{i}$ est le plongement induit $\left(\operatorname{avec} J_{0}=\left(i d, i_{0}\right)\right)$.

Soient pour $i \in \mathbb{N}$ :

- $m_{i, \varphi}$ la multiplicité de $Z_{i}$ au point $O_{i}$ (bien entendu si $O_{i} \notin Z_{i}$, $\left.m_{i, \varphi}=0\right)$;

- $H_{i, \varphi}$ la fonction de Hilbert-Samuel du germe $\left(Z_{i}, O_{i}\right)$ (bien entendu si $\left.O_{i} \notin Z_{i}, H_{i, \varphi}=0\right)$.

Soit maintenant $X=\left(X_{1}, \ldots, X_{n}\right)$ un système de coordonnées à l'origine de $\mathbb{K}^{n}$. Écrivons :

$$
\varphi(t)=\sum_{k=1}^{+\infty} A_{k} \cdot t^{k}, \quad A_{k} \in \mathbb{K}^{n}, \quad A_{k}=\left(a_{k}^{1}, \ldots, a_{k}^{n}\right) .
$$


L'écriture en coordonnées locales des transformations quadratiques, comme on le fait usuellement, fournit une suite d'isomorphismes locaux $\theta_{i}:(\mathbb{K}, 0) \times$ $\left(\mathbb{K}^{n}, 0\right) \rightarrow\left(E_{i}, O_{i}\right)$ telle que le diagramme $\left(D_{2}\right)$ suivant soit commutatif :

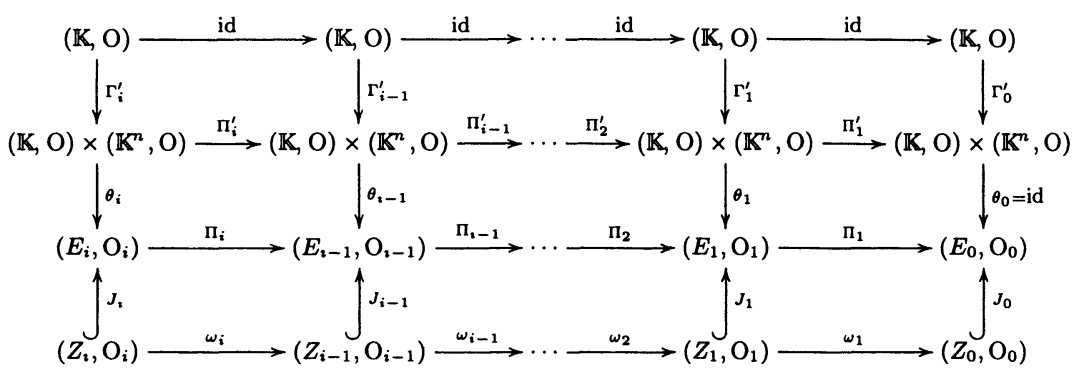

Via ces isomorphismes on a :

- $\forall i \geqslant 1, \Pi_{i}^{\prime}(t, X)=\left(t, t\left(A_{i}+X\right)\right)$ où $X=\left(X_{1}, \ldots, X_{n}\right)$;

- $\Gamma_{i}=\theta_{i} \circ \Gamma_{i}^{\prime}, \Gamma_{i}^{\prime}(t)=\left(t, \varphi_{i}(t)\right)$ où $\varphi_{i}(t)=\sum_{k>i} A_{k} \cdot t^{k-i} \in \mathcal{A}_{\left(\mathbb{K}^{n}, 0\right)}$.

On notera de plus par :

- $\left(Z_{i}^{\prime}, 0\right)$ le germe image de $\left(Z_{i}, O_{i}\right)$ par $\theta_{i}^{-1} \circ J_{i}$;

- $I_{i, A^{2}} \subset \mathbb{K}\left\{t, X_{1}, \ldots, X_{n}\right\}$ l'idéal définissant $\left(Z_{i}^{\prime}, 0\right)$, où $A^{i}=\left(A_{1}, \ldots, A_{i}\right)$ $\in \mathbb{K}^{n i}$.

Pour $i \in \mathbb{N}$, on note :

- $N_{i, \varphi}=N\left(I_{i, A^{2}}\right) \subset \mathbb{N}^{n+1}$ le diagramme des exposants initiaux de $I_{i, A^{2}}$ pour le système de coordonnées $(t, X)$. (Bien entendu si $O_{i} \notin Z_{i}$ i.e. $I_{i, A^{2}}=$ $\mathbb{K}\{t, X\}$, on pose $\left.N_{i, \varphi}=\mathbb{N}^{n+1}\right)$.

On fait alors la définition suivante :

DÉfinition 1.5. - Soient $(X, x)$ un germe de $\mathbb{K}$-espace analytique et un plongement $(X, x) \hookrightarrow\left(\mathbb{K}^{n}, 0\right)$. Pour $\varphi \in \mathcal{A}_{\left(\mathbb{K}^{n}, 0\right)}$, on appelle :

1) Suite des multiplicités de Nash de $(X, x)$ le long de $\varphi$ la suite:

$$
\mathcal{M}_{X, \varphi}=\left(m_{0, \varphi}, m_{1, \varphi}, \ldots, m_{i, \varphi}, \ldots\right)
$$


2) Suite de Nash des fonctions de Hilbert-Samuel de $(X, x)$ le long de $\varphi$ la suite :

$$
\mathcal{H}_{X, \varphi}=\left(H_{0, \varphi}, H_{1, \varphi}, \ldots, H_{i, \varphi}, \ldots\right)
$$

3) Suite de Nash des diagrammes des exposants initiaux de $(X, x)$ le long de $\varphi$ la suite :

$$
\mathcal{N}_{X, \varphi}=\left(N_{0, \varphi}, N_{1, \varphi}, \ldots, N_{i, \varphi}, \ldots\right)
$$

Pour le fait que le premier point de la définition précédente généralise la notion correspondante pour les hypersurfaces, nous renvoyons le lecteur à [H1] et [G-S-L-J] prop. 4.3 p. 18 et commentaires.

Ces définitions appellent quelques remarques. Tout d'abord pour $\varphi \in$ $\mathcal{A}_{(X, x)}$, les deux premières notions précédentes ne dépendent en rien du plongement. En effet, dans ce cas pour les définir, il suffit de considérer les $\Gamma_{i}$ comme des éléments de $\mathcal{A}_{\left(Z_{2}, O_{i}\right)}$ et de dire que les $\omega_{i}$ sont les éclatements de $Z_{i-1}$ de centre $O_{i-1}$. D'autre part, pour un $i$ donné, les tronqués jusqu'à l'ordre $i, \mathcal{M}_{X, \varphi^{2}}^{i}, \mathcal{H}_{X, \varphi^{2}}^{i}, \mathcal{N}_{X, \varphi^{2}}^{i}$, des suites précédentes ne dépendent, que du tronqué $\varphi^{i}$ à l'ordre $i$ de $\varphi$. C'est-à-dire du morphisme de $\mathbb{K}$-algèbre locale $\varphi^{i *}: \mathbb{K}\left\{X_{1}, \ldots, X_{n}\right\} \rightarrow \mathbb{K}\{t\} /(t)^{i+1}$ obtenu par passage au quotient. Finalement la motivation de la considération de tels diagrammes est la suivante. Soit $i \in \mathbb{N}^{*}$ et $\varphi \in \mathcal{A}_{\left(\mathbb{K}^{n}, 0\right)}$. Écrivons :

$$
\varphi(t)=\sum_{k=1}^{i} A_{k} t^{k}+t^{i} \varphi_{i}(t) \text { avec } A_{k} \in \mathbb{K}^{n}, \varphi_{i} \in \mathcal{A}_{\left(\mathbb{K}^{n}, 0\right)}
$$

Alors $\varphi \in \mathcal{A}_{(X, x)}$ si et seulement si l'arc $t \rightarrow\left(t, \varphi_{i}(t)\right)$ est dans $\mathcal{A}_{\left(Z_{i}^{\prime}, 0\right)}$ $\simeq \mathcal{A}_{\left(Z_{\imath}, O_{2}\right)}$. Aussi nous semble-t-il naturel d'étudier de tels objets, puisqu'ils sont les premières mesures de la singularité des $\left(Z_{i}^{\prime}, 0\right)$.

Nous donnons deux résultats dans cette direction. Le premier généralise en toute codimension le théorème 5 p. 154 de [L-J].

THÉORÈme 1.6. - Soient $(X, x) \hookrightarrow\left(\mathbb{K}^{n}, 0\right)$ et $\varphi \in \mathcal{A}_{\left(\mathbb{K}^{n}, O\right)}$.

1) La suite $\mathcal{M}_{X, \varphi}$ est décroissante et se stabilise à la valeur générique de la multiplicité de $X$, en un point $\varphi(t), t \neq 0$, assez petit.

2) La suite $\mathcal{H}_{X, \varphi}$ est décroissante (i.e. $\forall i, k \in \mathbb{N}, H_{i+1, \varphi}(k) \leqslant H_{i, \varphi}(k)$ ) et se stabilise à la valeur générique de la fonction de Hilbert-Samuel de $Z_{0}$, en un point $(t, \varphi(t)), t \neq 0$, assez petit. 
Notons que la fonction de Hilbert-Samuel de $Z_{0}$ en un point $(t, y)$ se calcule directement à partir de celle de $X$ en $y$. Nous essayerons au cours de la preuve de cet énoncé de préciser l'ordre auquel ces suites se stabilisent.

Les propriétés de généricité et de semi-continuité des applications $\varphi \rightarrow \mathcal{M}_{X, \varphi}, \varphi \rightarrow \mathcal{H}_{X, \varphi}$, et $\varphi \rightarrow \mathcal{N}_{X, \varphi}$ sont ensuite étudiées.

Pour cela, soit $i \in \mathbb{N}^{*}$, désignons par $\mathcal{A}_{(X, x)}^{i}$ l'ensemble des morphismes de $\mathbb{K}$-algèbre locale de $\mathcal{O}_{X, x} \rightarrow \mathbb{K}\{t\} /(t)^{i+1}$, et par $\Pi^{i}: \mathcal{A}_{(X, x)} \rightarrow \mathcal{A}_{(X, x)}^{i}$ le morphisme induit par la surjection canonique de $\mathbb{K}\{t\} \rightarrow \mathbb{K}\{t\} /(t)^{i+1}$. Tout choix de système de coordonnées sur $\mathbb{K}^{n}$ induit une identification de $\mathcal{A}_{\left(\mathbb{K}^{n}, 0\right)}^{i}$ avec $\mathbb{K}^{n i}$. Ordonnons ensuite, l'ensemble des suites d'entiers (resp. l'ensemble des suites de fonctions de $\mathbb{N}$ dans $\mathbb{N}$ ) par l'ordre lexicographique. De même l'ensemble $\mathcal{D}(n+1)$ des parties $N$ de $\mathbb{N}^{n+1}$ satisfaisant $N+\mathbb{N}^{n+1}=N$ étant muni de son ordre total naturel (cf. section 2), on ordonne l'ensemble des suites d'éléments de $\mathcal{D}(n+1)$ par l'ordre lexicographique. On a alors :

ThÉORÈme 1.7. - Soient $(X, x)$ et $i \in \mathbb{N}^{*}$. La fonction $\varphi^{i} \rightarrow \mathcal{N}_{X, \varphi^{2}}^{i}$ est semi-continue supérieurement pour la topologie de Zariski sur $\mathcal{A}_{\left(\mathbb{K}^{n}, 0\right)}^{i}$. C'est-à-dire, pour toute sous-variété algébrique irréductible $V$ de $\mathcal{A}_{\left(\mathbb{K}^{n}, 0\right)}^{i}$, il existe $V^{\prime}$ variété algébrique strictement incluse dans $V$ telle que :

1) $\forall \varphi^{i}, \theta^{i} \in V-V^{\prime}, \mathcal{N}_{X, \varphi^{2}}^{i}=\mathcal{N}_{X, \theta^{2}}^{i}$;

2) $\forall \varphi^{i} \in V-V^{\prime}, \forall \theta^{i} \in V^{\prime}, \mathcal{N}_{X, \varphi^{i}}^{i}<\mathcal{N}_{X, \theta^{i}}^{i}$.

Corollaire 1.8. - Soient $(X, x)$ et $i \in \mathbb{N}^{*}$. Il existe une partition :

$$
\overline{\Pi^{i}\left(\mathcal{A}_{(X, x)}\right)}=\bigcup_{1 \leqslant j \leqslant l_{i}} S_{i, j}
$$

où $S_{i, j}=U_{i, j}-W_{i, j}, U_{i, j}$ et $W_{i, j}$ sont des sous-variétés algébriques de $\mathcal{A}_{\left(\mathbb{K}^{n}, 0\right)}^{i}$ et $\mathcal{M}_{X, \varphi^{\imath}}^{i}, \mathcal{H}_{X, \varphi^{\imath}}^{i}, \mathcal{N}_{X, \varphi^{i}}^{i}$ sont constantes sur $S_{i, j}$. De plus pour $j \neq j^{\prime}$ les valeurs de $\mathcal{N}_{X, \varphi^{2}}^{i}$ sur $S_{i, j}$ et $S_{i, j^{\prime}}$ sont distinctes.

Les constructibles $\Pi^{i}\left(\mathcal{A}_{(X, x)}\right)$, dont l'étude fut initiée par J. Nash (cf. $[\mathrm{N}])$, portent donc une stratication naturelle, nous espérons que celle-ci pourra s'avérer utile à une meilleure compréhension de leurs géométries.

Comme première illustration de l'utilité de ces stratifications, nous définissons les notions de strates principales et de partie principale d'ordre $i$ de $\mathcal{A}_{(X, x)}$ 
(c.f. section 5, def. 5.1). Nous montrons comme conséquence élémentaire du théorème de semi-continuité comment le volume motivique de $\mathcal{A}_{(X, x)}$ (c.f. [D-L1]) se réalise comme «limite» des parties principales (c.f. th. 5.2). Nous traitons par cette technique quelques exemples.

Notre exposé est organisé comme suit. La section 2 rappelle quelques résultats et techniques que nous utiliserons par la suite. Ceux-ci sont issus de [B-M1], [B-M2], [T1], [T2]. Nous rappelons aussi quelques notations et définitions concernant la théorie de l'intégration motivique. Le paragraphe 3 prouve le Théorème 1.3. Notre schéma de preuve suit d'assez près celui de J.C. Tougeron dans le cas classique [T3]. La démonstration du Théorème 1.4 nécessitant quelques éléments sur la suite des multiplicités de Nash, les résultats et preuves concernant ces notions sont d'abord donnés dans la section 4. Les techniques de base proviennent du travail de E. Bierstone et P.D. Milman [B-M1]. La section 5 présente les notions de «strates principales et de partie principale d'ordre $i »$ de $\mathcal{A}_{(X, x)}$ et leur rapport avec le volume motivique tel que défini dans [D-L1]. Quelques exemples viennent illustrer cette technique. Le théorème 1.4 est démontré au paragraphe 6 . L'existence de désingularisation peut servir ici de substitut aux éléments provenant de la section 4. On perd alors en élémentarité mais aussi beaucoup d'informations concernant le diamètre des ouverts dont il est question dans l'énoncé 1.4.

\section{Rappels et notations}

Nous ferons un usage répété du résultat suivant. Nous l'appelerons par commodité théorème des fonctions implicites de Tougeron (cf. [T2]).

ThÉorÈme 2.1 (J.C. Tougeron). - Soient $x=\left(x_{1}, \ldots, x_{n}\right), y=$ $\left(y_{1}, \ldots, y_{p}\right), f=\left(f_{1}, \ldots, f_{q}\right) \in \mathbb{K}\{x, y\}^{q}$ telle que $f(0,0)=0$. Soit $I$ l'idéal engendré dans $\mathbb{K}\{x\}$ par les mineurs d'ordre $q$ de la matrice jacobienne $f_{y}^{\prime}(x, 0)=\left(\left(\partial f_{i} / \partial y_{j}\right)(x, 0)\right)$, et soit $I^{\prime}$ un idéal propre de $\mathbb{K}\{x\}$. Si $f(x, 0) \in \oplus_{q} I^{2} . I^{\prime}$, il existe $y(x) \in \oplus_{p} I . I^{\prime}$ tel que $f(x, y(x))=0$.

Le résultat est aussi valable en formel et en $C^{\infty}$.

Soit $\alpha=\left(\alpha_{1}, \ldots, \alpha_{n}\right) \in \mathbb{N}^{n}$, on notera $:|\alpha|=\alpha_{1}+\cdots+\alpha_{n} . \mathbb{N}^{n}$ est totalement ordonné par l'ordre lexicographique sur les $n+1$ upplets $\left(|\alpha|, \alpha_{1}, \ldots, \alpha_{n}\right)$. Si $A$ est un anneau commutatif unitaire intègre, et $f$ un élément non nul de $A[[X]]=A\left[\left[X_{1}, \ldots, X_{n}\right]\right]$, nous noterons par $\nu(f)$ son exposant initial. C'est-à-dire si :

$$
f=\sum_{\alpha \in \mathbb{N}^{n}} a_{\alpha} \cdot X^{\alpha}, \nu(f)=\operatorname{Min}\left\{\alpha \in \mathbb{N}^{n} \mid a_{\alpha} \neq 0\right\}
$$


Pour un idéal, $I \subset A[[X]]$, on notera $N_{I}$ le diagramme des exposants initiaux de $I$, i.e. :

$$
N_{I}=\left\{\alpha \in \mathbb{N}^{n} \mid \exists g \in I \text { tel que } \nu(g)=\alpha\right\} .
$$

On a : $N_{I}+\mathbb{N}^{n}=N_{I}$. Si $N \subset \mathbb{N}^{n}$ satisfait $N+\mathbb{N}^{n}=N$, le lemme de Dickson assure l'existence d'une partie finie unique de $\mathbb{N}^{n},\left\{\alpha^{1}, \ldots, \alpha^{p}\right\}$, telle que :

$$
N=\cup_{1 \leqslant i \leqslant p}\left(\alpha^{i}+\mathbb{N}^{n}\right) \text { et } \alpha^{j} \notin \cup_{i \neq j}\left(\alpha^{i}+\mathbb{N}^{n}\right)
$$

Les $\alpha^{i}$ sont dits les sommets de $N$. L'ensemble $\mathcal{D}(n)$ des parties de $\mathbb{N}^{n}$ stables par translations (i.e. satisfaisant $N+\mathbb{N}^{n}=N$ ) est totalement ordonné comme suit. Soit $N_{1}, N_{2} \in \mathcal{D}(n)$. Pour chaque $i=1,2$, soient $\beta_{i}^{k}, k=1, \ldots, t_{i}$ les sommets de $N_{i}$ indexés dans l'ordre croissant. Après avoir éventuellement permuté $N_{1}$ et $N_{2}$, il existe $t \in \mathbb{N}$ tel que : $\beta_{1}^{k}=\beta_{2}^{k}$, $1 \leqslant k \leqslant t$ et (1) $t_{1}=t=t_{2},(2) t_{1}>t=t_{2}$ ou bien (3) $t_{1}, t_{2}>t$ et $\beta_{t+1}^{1}<\beta_{t+1}^{2}$. Dans le cas (1), $N_{1}=N_{2}$. Dans les cas (2) et (3), $N_{1}<N_{2}$. Il revient au même de dire que la suite $\left(\beta_{1}^{1}, \ldots, \beta_{1}^{t_{1}}, \infty, \ldots\right)$ est strictement plus petite que la suite $\left(\beta_{2}^{1}, \ldots, \beta_{2}^{t_{2}}, \infty, \ldots\right)$ pour l'ordre lexicographique, avec la convention $\beta<\infty$ pour tout $\beta \in \mathbb{N}^{n}$.

Si $N$ est un élément de $\mathcal{D}(n)$, on notera $H_{N}$ la fonction de $\mathbb{N}$ dans $\mathbb{N}$ définie par :

$$
H_{N}(k)=\operatorname{card}\left\{\alpha \in \mathbb{N}^{n} \mid \alpha \notin N \text { et }|\alpha| \leqslant k\right\} .
$$

Soit I un idéal de $\mathbb{K}[[X]]$ et $g_{1}, \ldots, g_{p}$ des éléments de $I$ tels que $\nu\left(g_{1}\right), \ldots, \nu\left(g_{p}\right)$ sont les sommets de $N(I)$. Nous disons que $g_{1}, \ldots, g_{p}$ est une base standard de $I$. Il existe par ailleurs une unique famille $g_{1}, \ldots, g_{p}$ telle que :

$$
\begin{gathered}
\operatorname{InMon}\left(g_{i}\right)=X^{\beta^{2}} \\
\operatorname{Supp}\left(X^{\beta^{2}}-g_{i}\right) \subset \mathbb{N}^{n}-N(I), \quad \forall 1 \leqslant i \leqslant p,
\end{gathered}
$$

où $\beta^{i}, \ldots, \beta^{p}$ sont les sommets de $N(I)$, Supp désigne le support d'un élément de $\mathbb{K}[[X]]$, InMon $(f)=a_{\nu(f)} X^{\nu(f)}$. Une telle famille est appelée la base standard distinguée de $I$. Si :

$$
H_{I}(k)=\operatorname{dim}_{\mathbb{K}} \frac{\mathbb{K}[[X]]}{I+\mathcal{M}^{k+1}}
$$

désigne la fonction de Hilbert-Samuel de $I$, on a :

$$
H_{I}(k)=\operatorname{card}\left\{\alpha \in \mathbb{N}^{n}-N(I)|| \alpha \mid \leqslant k\right\}=H_{N(I)}(k) .
$$

Pour plus d'informations, nous renvoyons le lecteur à [B-M2]. Nous ferons par ailleurs un usage libre de la notion de transformé strict d'un idéal de 
$\mathbb{K}[[X]]$. Rappelons simplement que si $g_{1}, \ldots, g_{p}$ est une base standard de $I$, alors leurs transformées strictes $g_{1}^{\prime}, \ldots, g_{p}^{\prime}$, par l'éclatement de centre l'origine engendrent le transformé strict $I^{\prime}$ de $I$.

Dans le texte de l'article, nous considèrons des anneaux de la forme $A[[t, X]], X=\left(X_{1}, \ldots, X_{n}\right)$. Lorsque nous parlons de diagramme des exposants initiaux $\subset \mathbb{N}^{n+1}$ d'un idéal $I$ de $A[[t, X]]$, nous considérons toujours des multi-indices $\left(\alpha_{0}, \alpha_{1}, \ldots, \alpha_{n}\right)$ où le premier indice $\alpha_{0}$ correspond toujours à la variable $t$.

Si X est un espace analytique et $\varphi \in \mathcal{A}_{X}$, nous noterons par $\mathcal{B}_{X, i}(\varphi)$ la boule :

$$
\mathcal{B}_{X, i}(\varphi)=\left\{\varphi^{\prime} \in \mathcal{A}_{X} / \mathcal{D}_{X}\left(\varphi, \varphi^{\prime}\right)<e^{-i}\right\}=\left\{\varphi^{\prime} \in \mathcal{A}_{X} / \operatorname{ord}\left(\varphi-\varphi^{\prime}\right)>i\right\} .
$$

Lorsque le contexte le permettra, nous omettrons l'indice $X$.

Rappelons que l'on désigne par $K_{0}\left(\mathcal{V}_{\mathbb{K}}\right)$ l'anneau de Grothendieck des $\mathbb{K}$-variétés (i.e. les schémas réduits séparés de type fini sur $\mathbb{K}$ ). Par définition $K_{0}\left(\mathcal{V}_{\mathbb{K}}\right)$ est l'anneau engendré par les symboles $[V]$, pour une $\mathbb{K}$-variété $V$, modulo les relations :

- $[V]=\left[V^{\prime}\right]$, si $V$ et $V^{\prime}$ sont $\mathbb{K}$-isomorphes

$-[V]=\left[V^{\prime}\right]+\left[V-V^{\prime}\right]$, si $V^{\prime}$ est fermé dans $[V]$

$-[V] \times\left[V^{\prime}\right]=\left[V \times_{\mathbb{K}} V^{\prime}\right]$.

La classe de la droite affine $\mathbb{A}_{\mathbb{K}}^{1}$ sera notée $\mathbb{L}$. Suivant les notations de Looijenga $[\mathrm{Lo}], \mathcal{M}_{\mathbb{K}}$ désignera l'anneau $\mathbb{K}_{0}\left(\mathcal{V}_{\mathbb{K}}\right)\left[\mathbb{L}^{-1}\right]$ (ce qui correspond à la notation $\mathcal{M}_{\text {loc }}$ de [D-L1]). Pour $m \in \mathbb{Z}$, on note $F^{m} \mathcal{M}_{\mathbb{K}}$ le sous-groupe engendré par les symboles $[S] \mathbb{L}^{i}$ où $i-\operatorname{dim} S \geqslant m$. On notera $\widehat{\mathcal{M}}_{\mathbb{K}}$ le complété de $\mathcal{M}_{\mathbb{K}}$ par rapport à cette filtration et $\overline{\mathcal{M}}_{\mathbb{K}}$ l'image de $\mathcal{M}_{\mathbb{K}}$ par l'application

naturelle de $\mathcal{M}_{\mathbb{K}}$ dans $\widehat{\mathcal{M}}_{\mathbb{K}}$. Concernant les mesures motiviques de [D-L1], nous adoptons la convention de [Lo], i.e. les mesures de Denef-Loeser sont multipliées par $\mathbb{L}^{d}, d$ désignant la dimension de la variété ou du germe que l'on considère.

\section{Inégalités de Lojasiewicz fibrées au niveau de l'espace des arcs}

Nous commençons d'abord par constater qu'il suffit d'obtenir le Théorème 1.3, lorsque $X$ et $Y$ sont respectivement des ouverts $\Omega_{n}$ et $\Omega_{p}$ de $\mathbb{K}^{n}$ et $\mathbb{K}^{p}$.

En effet, étant donné un point $(x, y) \in X \times Y$, il suffit d'établir l'existence de voisinages $V_{x}$ et $V_{y}$ et d'un $\alpha \geqslant 0$ tels que la propriété de 1.3 soit valide pour tout $\left(\phi_{0}, \varphi\right) \in \mathcal{A}_{X, V_{x}} \times \mathcal{A}_{Y, V_{y}}$. On peut par conséquent supposer que 
$X$ et $Y$ sont des modèles locaux, $X$ défini par $f_{1}, \ldots, f_{k}$, analytiques sur un ouvert $\Omega_{n}$, et que le morphisme $F$ est induit par des fonctions analytiques $F_{1}, \ldots, F_{p}$ sur $\Omega_{n}$. Considérons alors le morphisme $G$ :

$$
\begin{aligned}
& G: \Omega_{n} \rightarrow \Omega_{p} \times \mathbb{K}^{k} \\
& x \rightarrow\left(F_{1}(x), \ldots, F_{p}(x), f_{1}(x), \ldots, f_{k}(x)\right) .
\end{aligned}
$$

Si le Théorème 1.3 est valide pour le morphisme $\mathcal{G}$, il l'est pour $\mathcal{F}$. En effet, si $\varphi \in \mathcal{A}_{Y} \subset \mathcal{A}_{\Omega_{p}}$, considérons l'arc $\theta=(\varphi, 0) \in \mathcal{A}_{\Omega_{p} \times \mathbb{K}^{k}}$. On a pour tout $\phi \in \mathcal{A}_{X} \subset \mathcal{A}_{\Omega_{n}}$ :

$$
\begin{gathered}
\mathcal{D}_{Y}(\mathcal{F}(\phi), \varphi)=\mathcal{D}_{\Omega_{p} \times \mathbb{K}^{k}}(\mathcal{G}(\phi), \theta) \\
\mathcal{D}_{X}\left(\phi, \mathcal{F}^{-1}(\varphi)\right)=\mathcal{D}_{\Omega_{n}}\left(\phi, \mathcal{G}^{-1}(\theta)\right) .
\end{gathered}
$$

Nous établissons maintenant 1.3 dans la situation citée plus haut. Notre schéma de démonstration suit, tout en l'adaptant à notre cas, celui de J.C. Tougeron [T3]. Pour cela désignons par $\mathcal{O}$ le faisceau des germes de fonctions $\mathbb{K}$-analytiques sur $\Omega_{n} \times \Omega_{p}$ et $\mathcal{I} \subset \mathcal{O}$ un sous-faisceau cohérent d'idéaux. Nous utiliserons les notations suivantes :

- $V(\mathcal{I})$ l'espace analytique de zéros de $\mathcal{I}$, i.e. $V(\mathcal{I})=(|X|,(\mathcal{O} / \mathcal{I})|| X \mid)$ où $|X|=\operatorname{Support}(\mathcal{O} / \mathcal{I})$;

- $\mathcal{V}(\mathcal{I})=\mathcal{A}_{V(\mathcal{I})}$

- Soit $\varphi \in \mathcal{A}_{\Omega_{p}}, \mathcal{V}\left(\varphi^{*} \mathcal{I}\right)=\left\{\phi \in \mathcal{A}_{\Omega_{n}} \mid(\phi, \varphi) \in \mathcal{V}(\mathcal{I})\right\}$;

- Si $\left(x_{0}, y_{0}\right) \in \Omega_{n} \times \Omega_{p}$, on désignera par $\mathcal{I}_{\left(x_{0}, y_{0}\right)}$ la fibre de $\mathcal{I}$ en $\left(x_{0}, y_{0}\right)$ et par $f_{1}(x, y), \ldots, f_{k_{0}}(x, y)$ un système de générateurs de $\mathcal{I}_{\left(x_{0}, y_{0}\right)}$;

- Si $\theta=\left(\theta_{1}, \ldots, \theta_{s}\right) \in \mathcal{O}_{1}^{s}$, on pose : $|\theta|=\left|\theta_{1}\right|+\ldots+\left|\theta_{s}\right|$, où $\left|\theta_{j}\right|=$ $e^{-\operatorname{ord}\left(\theta_{j}\right)}$.

Le Théorème 1.3 découle alors du résultat suivant, appliqué au sousfaisceau de $\mathcal{O}$ engendré par $F_{1}(x)-y_{1}, \ldots, F_{p}(x)-y_{p}$, où $F_{1}, \ldots, F_{p}$ sont les composantes du morphisme $F: \Omega_{n} \rightarrow \Omega_{p}$.

THÉORÈME 3.1. - Soient $\mathcal{I} \subset \mathcal{O}$ et $K, K^{\prime}$ deux compacts comme précédemment. Il existe alors $\alpha \geqslant 0$ tel que :

$\forall \varphi \in \mathcal{A}_{\left(\Omega_{p}, K^{\prime}\right)}, \forall \phi_{0} \in \mathcal{A}_{\left(\Omega_{n}, K\right)}, \exists C_{\left(\phi_{0}, \varphi\right)}>0$ et un voisinage $\mathcal{V}_{\phi_{0}}$ de $\phi_{0}$ satisfaisant :

$$
\forall \phi \in \mathcal{V}_{\phi_{0}}, \sum_{i=1}^{k_{0}}\left|f_{i}(\phi, \varphi)\right| \geqslant C_{\left(\phi_{0}, \varphi\right)} \mathcal{D}\left(\phi, \mathcal{V}\left(\varphi^{*} \mathcal{I}\right)\right)^{\alpha}
$$

où $f_{1}(x, y), \ldots, f_{k_{0}}(x, y)$ engendrent $\mathcal{I}_{\left(\phi_{0}(0), \varphi(0)\right)}$. 
Géométrie de l'espace des arcs tracés sur un espace analytique

Preuve. - Pour prouver le résultat, il suffit de voir que pour un point $\left(x_{0}, y_{0}\right) \in \Omega_{n} \times \Omega_{p}$, il existe des voisinages $V_{x_{0}}, W_{y_{0}}$ de $x_{0}$ et $y_{0}$, et un $\alpha \geqslant 0$ tels que la propriété de 3.1 soit satisfaite pour $\left(\phi_{0}, \varphi\right) \in \mathcal{A}_{V_{x_{0}}} \times \mathcal{A}_{W_{y_{0}}} . \mathrm{Si}$ $\left(x_{0}, y_{0}\right) \notin V(\mathcal{I})$, ceci est trivialement satisfait avec $\alpha=0$. Nous supposerons donc $\left(x_{0}, y_{0}\right)=(0,0) \in V(\mathcal{I})$. Pour prouver l'assertion précédente, il suffit de voir que :

(3.2) il existe un voisinage $V_{0}$ de 0 dans $\mathbb{K}^{p}$ et un $\alpha \geqslant 0$ satisfaisant : $\forall \varphi \in \mathcal{A}_{V_{0}}$ tel que $(0, \varphi) \in \mathcal{V}(\mathcal{I}), \exists C_{\varphi}>0$ et un voisinage $\mathcal{V}_{0}$ de 0 dans $\mathcal{A}_{\left(\mathbb{K}^{n}, 0\right)}$ tel que :

$$
\forall \phi \in \mathcal{V}_{0}, \sum_{i=1}^{k_{0}}\left|f_{i}(\phi, \varphi)\right| \geqslant C_{\varphi} \mathcal{D}\left(\phi, \mathcal{V}\left(\varphi^{*} \mathcal{I}\right)\right)^{\alpha} .
$$

En effet supposons (3.2) prouvée pour tout faisceau cohérent d'idéaux. Partant d'un faisceau cohérent d'idéaux $\mathcal{I}$ engendré par $f_{1}(x, y), \ldots, f_{k_{0}}(x, y)$ sur un voisinage de l'origine dans $\mathbb{K}^{n} \times \mathbb{K}^{p}$, on considère le faisceau d'idéaux $\mathcal{J}$ engendré sur un voisinage de l'origine dans $\mathbb{K}^{n} \times \mathbb{K}^{n+p}$ par :

$$
G_{1}(x, z, y)=f_{1}(x+z, y), \ldots, G_{k_{0}}(x, z, y)=f_{k_{0}}(x+z, y) .
$$

L'application de (3.2) à $\mathcal{J}$ fournit un voisinage $U_{0}=V_{0} \times W_{0}$ de 0 dans $\mathbb{K}^{n+p}=\mathbb{K}^{n} \times \mathbb{K}^{p}$ et un $\alpha \geqslant 0$ satisfaisant :

(3.3) $\forall\left(\phi_{0}, \varphi\right) \in \mathcal{A}_{U_{0}}=\mathcal{A}_{V_{0}} \times \mathcal{A}_{W_{0}}$ tel que $\left(0, \phi_{0}, \varphi\right) \in \mathcal{V}(\mathcal{J})$,

$\exists C_{\left(\phi_{0}, \varphi\right)}$ et un voisinage $\mathcal{V}_{0}$ de 0 dans $\mathcal{A}_{\left(\mathbb{K}^{n}, 0\right)}$ tels que :

$$
\forall \theta \in \mathcal{V}_{0}, \sum_{i=1}^{k_{0}}\left|G_{i}\left(\theta, \phi_{0}, \varphi\right)\right| \geqslant \mathcal{C}_{\phi_{0}, \varphi} \mathcal{D}\left(\theta, \mathcal{V}\left(\left(\phi_{0}, \varphi\right)^{*} \mathcal{J}\right)^{\alpha} .\right.
$$

Soient alors $\varphi \in \mathcal{A}_{V_{0}}$ et $\phi_{0} \in \mathcal{A}_{W_{0}}$. Si $\left(\phi_{0}, \varphi\right) \notin \mathcal{V}(\mathcal{I})$, posons :

$$
s=\operatorname{Min}\left(\operatorname{ordf} f_{i}\left(\phi_{0}, \varphi\right)\right) \mathcal{C}_{\left(\phi_{0}, \varphi\right)}=e^{-s} \text { et } \mathcal{V}_{\phi_{0}}=\mathcal{B}\left(\phi_{0}, e^{-s}\right)
$$

On a alors :

$$
\forall \phi \in \mathcal{V}_{\phi_{0}}, \sum_{j=1}^{k_{0}}\left|f_{j}(\phi, \varphi)\right| \geqslant \mathcal{C}_{\left(\phi_{0}, \varphi\right)} \geqslant \mathcal{C}_{\left(\phi_{0}, \varphi\right)} \mathcal{D}\left(\phi, \mathcal{V}\left(\varphi^{*} \mathcal{I}\right)\right)^{\alpha} .
$$

Si maintenant $\left(\phi_{0}, \varphi\right) \in \mathcal{V}(\mathcal{I})$, alors $\left(0, \phi_{0}, \varphi\right) \in \mathcal{V}(\mathcal{J})$. D'où l'existence d'un voisinage $\mathcal{V}_{0}$ de 0 dans $\mathcal{A}_{\left(\mathbb{K}^{n}, 0\right)}$ et d'une constante $\mathcal{C}_{\left(\phi_{0}, \varphi\right)}$ comme dans (3.3). 
Posons : $\mathcal{V}_{\phi_{0}}=\phi_{0}+\mathcal{V}_{0}$. Puisque :

$$
\begin{gathered}
\sum_{i=1}^{k_{0}}\left|f_{i}(\phi, \varphi)\right|=\sum_{i=1}^{k_{0}}\left|G_{i}\left(\phi-\phi_{0}, \phi_{0}, \varphi\right)\right| \\
\mathcal{D}\left(\phi, \mathcal{V}\left(\varphi^{*} \mathcal{I}\right)\right)=\mathcal{D}\left(\phi-\phi_{0}, \mathcal{V}\left(\left(\phi_{0}, \varphi\right)^{*} \mathcal{J}\right) .\right.
\end{gathered}
$$

On a :

$$
\forall \phi \in \mathcal{V}_{\phi_{0}}, \sum_{i=1}^{k_{0}}\left|f_{i}(\phi, \varphi)\right| \geqslant C_{\left(\phi_{0}, \varphi\right)} \mathcal{D}\left(\phi, \mathcal{V}\left(\varphi^{*} \mathcal{I}\right)\right)^{\alpha} .
$$

Ce qui est l'assertion désirée. Pour prouver (3.2) nous laissons le soin au lecteur de vérifier que celle-ci est équivalente à :

(3.4) $\exists V_{0}$ voisinage de 0 dans $\mathbb{K}^{p}$ et un $\alpha \geqslant 0$ satisfaisant : $\forall \varphi \in \mathcal{A}_{V_{0}}$ tel que $(0, \varphi) \in \mathcal{V}(\mathcal{I})$, il existe $a_{\varphi}, k_{\varphi} \in \mathbb{N}$ tels que l'implication suivante soit vérifiée :

$\forall i \in \mathbb{N}, \forall \phi \in \mathcal{A}_{\left(\mathbb{K}^{n}, 0\right)},\left(\operatorname{ord}(\phi)>k_{\varphi}\right.$ et $\left.\operatorname{Min}_{1 \leqslant j \leqslant k_{0}} \operatorname{ord}\left(f_{j}(\phi, \varphi)\right)>a_{\varphi}+\alpha i\right)$

$$
\Longrightarrow\left(\exists \phi^{\prime} \in \mathcal{A}_{\left(\mathbb{K}^{n}, 0\right)}, \phi^{\prime} \in \mathcal{V}\left(\varphi^{*} \mathcal{I}\right) \text { et } \operatorname{ord}\left(\phi-\phi^{\prime}\right)>i\right) \text {. }
$$

Nous allons à présent prouver (3.4) par récurrence descendante sur la hauteur de $\mathcal{I}_{(0,0)}$.

Si $\operatorname{haut}\left(\mathcal{I}_{(0,0)}\right)=n+p$, quitte à nous restreindre à un voisinage assez petit de $(0,0)$ dans $\mathbb{K}^{n} \times \mathbb{K}^{p}$, on a $V(\mathcal{I})=(0,0)$ et $\exists m \in \mathbb{N}^{*}$ tel que : $\left(x_{1}, \ldots, x_{n}, y_{1}, \ldots, y_{p}\right)^{m} \subset \mathcal{I}_{(0,0)}$. Par suite (3.4) est satisfaite avec $\alpha=m$, $a_{0}=k_{0}=0$.

Soit maintenant $\mathcal{I}$ tel que $\operatorname{haut}\left(\mathcal{I}_{(0,0)}\right)=r<n+p$. Il existe un entier $m$ et $\mathcal{P}^{1}, \ldots, \mathcal{P}^{s}$, des sous-faisceaux cohérents d'idéaux de $\mathcal{O}$ dans un voisinage de $(0,0)$ dans $\mathbb{K}^{n} \times \mathbb{K}^{p}$ tels que :

$$
\begin{gathered}
\forall j, 1 \leqslant j \leqslant s, \mathcal{P}_{(0,0)}^{j} \text { est premier de hauteur } \geqslant r ; \\
\exists m \in \mathbb{N} \mid\left(\bigcap_{1 \leqslant j \leqslant s} \mathcal{P}^{j}\right)^{m} \subset \mathcal{I} \subset \bigcap_{1 \leqslant j \leqslant s} \mathcal{P}^{j} .
\end{gathered}
$$

Supposons que pour tout $j, 1 \leqslant j \leqslant s,(3.4)$ soit satisfaite pour $\mathcal{P}^{j}$ avec une constante $\alpha_{j} \geqslant 0$. Il est alors aisé de vérifier que (3.4) est satisfaite pour $\mathcal{I}$ avec $\alpha=m\left(\sum_{j=1}^{s} \alpha_{j}\right)$. En effet, soit $(0, \varphi) \in \mathcal{V}(\mathcal{I})$. Désignons par $\Delta_{\varphi}$ l'ensemble (nécessairement non vide) :

$$
\Delta_{\varphi}=\left\{j \in\{1, \ldots, s\} \mid(0, \varphi) \in \mathcal{V}\left(\mathcal{P}^{j}\right)\right\}
$$


Considérons aussi $\bar{\Delta}_{\varphi}=\{1, \ldots, s\}-\Delta_{\varphi}$. On note alors :

- $a_{\varphi}^{j}=\operatorname{ord}\left((0, \varphi)^{*} \mathcal{P}^{j}\right)$, si $j \in \bar{\Delta}_{\varphi}$

- $k_{\varphi}^{j}, a_{\varphi}^{j}$ les entiers associés à $\mathcal{P}^{j} \operatorname{par}(3.4)$, si $j \in \Delta_{\varphi}$.

Soient alors : $k_{\varphi}=\operatorname{Max}\left(\operatorname{Max}_{j \in \bar{\Delta}_{\varphi}} a_{\varphi}^{j}, \operatorname{Max}_{j \in \Delta_{\varphi}} k_{\varphi}^{j}\right)$ et $a_{\varphi}=m\left(\sum_{j=1}^{s} a_{\varphi}^{j}\right)$. Si $\phi$ est tel que $\operatorname{ord}(\phi)>k_{\varphi}$ et $\operatorname{Min}_{1 \leqslant l \leqslant k_{0}} \operatorname{ord}\left(f_{l}(\phi, \varphi)\right)>a_{\varphi}+\alpha i$, il existe $j \in \Delta_{\varphi}$ tel que $\operatorname{ord}\left((\phi, \varphi)^{*} \mathcal{P}^{j}\right)>a_{\varphi}^{j}+\alpha_{j} i$. D'où l'assertion désirée pour $\mathcal{I}$.

Par suite, nous supposerons que $\mathcal{I}$ est tel que $\mathcal{I}_{(0,0)}$ est premier de hauteur $r<n+p$. Soit maintenant $J$ l'idéal des éléments de $\mathbb{K}\left\{y_{1}, \ldots, y_{p}\right\}$ nuls sur le germe en $(0,0)$ de $V(\mathcal{I}) \cap\left(0 \times \mathbb{K}^{p}\right)$. On peut supposer $J \subset \mathcal{I}_{(0,0)}$. En effet, si ce n'était pas le cas, considérons $h \in J, h \notin \mathcal{I}_{(0,0)}$. Soit alors $\mathcal{J}$ le sous-faisceau cohérent d'idéaux de $\mathcal{O}$ engendré sur un voisinage de $(0,0)$ par $h$ et $\mathcal{I}_{(0,0)}$. On a alors $\operatorname{haut}\left(\mathcal{J}_{(0,0)}\right)>r$.

Donc par hypothèse de récurrence, il existe un voisinage $V_{0}$ de 0 dans $\mathbb{K}^{p}$ tel que (3.4) soit vérifiée pour $\mathcal{J}$. On peut toujours supposer que $h$ est nul sur $V(\mathcal{I}) \cap\left(0 \times V_{0}\right)$. Mais alors pour $\varphi \in \mathcal{A}_{V_{0}}$ :

$$
\begin{aligned}
& -(0, \varphi) \in \mathcal{V}(\mathcal{I}) \Longleftrightarrow(0, \varphi) \in \mathcal{V}(\mathcal{J}) ; \\
& -\mathcal{V}\left(\varphi^{*} \mathcal{I}\right)=\mathcal{V}\left(\varphi^{*} \mathcal{J}\right), \text { pour } \varphi \text { tel que }(0, \varphi) \in \mathcal{V}(\mathcal{I}) ; \\
& -\forall \phi \in \mathcal{A}_{\left(\mathbb{K}^{n}, 0\right)}, \forall \varphi \in \mathcal{A}_{V_{0}} \text { tel que }(0, \varphi) \in \mathcal{V}(\mathcal{I}), \\
& \operatorname{Min}_{1 \leqslant j \leqslant k_{0}}\left(\operatorname{ord}\left(f_{j}(\phi, \varphi)\right)=\operatorname{Min}\left(\operatorname{ord}(h(\varphi)), \operatorname{ord}\left(f_{j}(\phi, \varphi)\right), 1 \leqslant j \leqslant k_{0}\right) .\right.
\end{aligned}
$$

Il en résulte que (3.4) est aussi vérifiée pour $\mathcal{I}$ avec le même $\alpha \geqslant 0$ et le même $V_{0}$. Nous supposerons donc que $J \subset \mathcal{I}_{(0,0)}$ et que cette inclusion est stricte (sans quoi (3.4) est triviale). On a alors le lemme suivant :

Lemme 3.2 ( [T3], Lemme 2.4). - Supposons $J \varsubsetneqq \mathcal{I}_{(0,0)}$. Soit $r-l$ la hauteur de l'idéal premier J. Après une éventuelle permutation sur les coordonnées $x_{1}, \ldots, x_{n}$, il existe $u_{1}(x, y), \ldots, u_{l}(x, y) \in \mathcal{I}_{(0,0)}$ tels que :

$$
u(x, y)=\operatorname{det}\left(\frac{\partial u_{k}(x, y)}{\partial x_{j}}\right)_{1 \leqslant k, j \leqslant l} \notin \mathcal{I}_{(0,0)} .
$$


Puisque $\operatorname{haut}(J)=r-l$ et $J$ est premier, il existe $u_{l+1}(y), \ldots, u_{r}(y) \in J$ tels qu' après une éventuelle permutation sur les coordonnées $y_{1}, \ldots, y_{p}$ :

$$
v(y)=\operatorname{det}\left(\frac{\partial u_{l+k}(y)}{\partial y_{j}}\right)_{1 \leqslant k, j \leqslant r-l} \notin J .
$$

Par suite :

$$
v(y) u(x, y)=\operatorname{det}\left(\frac{\partial u_{k}(x, y)}{\partial z_{j}}\right)_{1 \leqslant k, j \leqslant r} \notin \mathcal{I}_{(0,0)},
$$

où $z_{j}=x_{j}, 1 \leqslant j \leqslant l$ et $z_{j}=y_{j-l}, l+1 \leqslant j \leqslant r$. Par le critère jacobien des points simples, le localisé $\left(\mathcal{O}_{(0,0)}\right)_{\mathcal{I}_{(0,0)}}$ est régulier de dimension $r$ et son maximal est engendré par $u_{1}, \ldots, u_{r}$. Par conséquent, il existe $w(x, y) \in$ $\left.\mathcal{O}_{(0,0)}\right) \backslash \mathcal{I}_{(0,0)}$ tel que : $w \cdot \mathcal{I}_{(0,0)} \subset\left(u_{1}, \ldots, u_{r}\right) \mathcal{O}_{(0,0)}$.

Soit $\Delta=u(x, y) v(y) w(x, y)$. Considérons maintenant $\mathcal{J}$ le sous-faisceau cohérent d'idéaux de $\mathcal{O}$ engendré par $\mathcal{I}$ et $\Delta$ sur un voisinage de 0 dans $\mathbb{K}^{n} \times \mathbb{K}^{p}$, suffisamment petit pour que :

$$
\Delta \cdot \mathcal{I} \subset\left(u_{1}, \ldots, u_{r}\right) \cdot \mathcal{O} .
$$

Puisque $\operatorname{haut}\left(\mathcal{J}_{(0,0}\right) \geqslant r+1$, l'hypothèse de récurrence nous assure l'existence d'un voisinage $V_{0}$ de 0 dans $\mathbb{K}^{p}$ et d'un $\alpha \geqslant 0$ tel que (3.4) soit satisfaite pour $\mathcal{J}$. Nous allons montrer que (3.4) est valide pour $\mathcal{I}$ avec le même voisinage $V_{0}$ et avec $\beta=\operatorname{Max}(2 \alpha, 1)$.

Soit $\varphi \in \mathcal{A}_{V_{0}}$ tel que $(0, \varphi) \in \mathcal{V}(\mathcal{I})$. Deux cas peuvent se présenter :

$$
\underline{1^{\text {er }} \text { cas : }} \quad(0, \varphi) \notin \mathcal{V}(\mathcal{J}) .
$$

Soit alors $k_{\varphi}=\operatorname{ord}(\Delta(0, \varphi))<+\infty$. Posons $a_{\varphi}=2 k_{\varphi}$. Considérons alors, $\phi \in \mathcal{A}_{\left(\mathbb{K}^{n}, 0\right)}$ tel que $\operatorname{ord}(\phi)>k_{\varphi}$ et $\operatorname{Min}_{1 \leqslant j \leqslant k_{0}} \operatorname{ord}\left(f_{j}(\phi, \varphi)\right)>a_{\varphi}+i$. On a :

$$
\begin{aligned}
& -\operatorname{ord}(\Delta(\phi, \varphi))=\operatorname{ord}(\Delta(0, \varphi))=k_{\varphi}, \text { en particulier } \operatorname{ord}(u(\phi, \varphi)) \leqslant k_{\varphi} \\
& -\operatorname{Min}_{1 \leqslant j \leqslant r} \operatorname{ord}\left(u_{j}(\phi, \varphi)\right) \geqslant \operatorname{Min}_{1 \leqslant j \leqslant k_{0}}\left(\operatorname{ord}\left(f_{j}(\phi, \varphi)\right)>2 k_{\varphi}+i .\right.
\end{aligned}
$$

Considérons alors les $l$ éléments $U_{1}\left(t, z_{1}, \ldots, z_{l}\right), \ldots, U_{l}\left(t, z_{1}, \ldots, z_{l}\right)$ de $\mathbb{K}\left\{t, z_{1}, \ldots, z_{l}\right\}$ suivants :

$$
\begin{aligned}
& U_{j}\left(t, z_{1}, \ldots, z_{l}\right)=u_{j}\left(\phi_{1}(t)+z_{1}, \ldots, \phi_{l}(t)+z_{l}, \phi_{l+1}(t), \ldots, \phi_{n}(t), \varphi(t)\right), 1 \leqslant j \leqslant l . \\
& U_{j}(0,0)=0,1 \leqslant j \leqslant l .
\end{aligned}
$$


Alors :

$$
\operatorname{det}\left(\frac{\partial U_{k}}{\partial z_{j}}(t, 0)\right)_{1 \leqslant k, j \leqslant l}=u(\phi(t), \varphi(t))
$$

Par suite :

$$
U_{j}(t, 0)=u_{j}(\phi(t), \varphi(t)) \in\left(\operatorname{det}\left(\frac{\partial U_{k}}{\partial z_{j}}(t, 0)\right)^{2} \cdot t^{i+1+2\left(k_{\varphi}-v a l u(\phi, \varphi)\right)}\right) .
$$

Par conséquent le théorème des fonctions implicites de Tougeron donne l'existence de $\theta_{1}, \ldots, \theta_{l} \in \mathbb{K}\{t\}$ tels que :

$$
\begin{aligned}
& \theta_{j}(t) \in \operatorname{det}\left(\frac{\partial U_{k}}{\partial z_{j}}(t, 0)\right) \cdot(t)^{i+1+2\left(k_{\varphi}-\operatorname{valu}(\phi, \varphi)\right)} \subset(t)^{i+1+k_{\varphi}+k_{\varphi}-\operatorname{valu}(\phi, \varphi)} \\
& \subset(t)^{i+1+k_{\varphi}} \\
& U_{j}\left(t, \theta_{1}(t), \ldots, \theta_{l}(t)\right)=0,1 \leqslant j \leqslant l .
\end{aligned}
$$

Posons alors $\phi^{\prime}=\left(\phi_{1}+\theta_{1}, \ldots, \phi_{l}+\theta_{l}, \phi_{l+1}, \ldots, \phi_{n}\right)$.

On a :

$$
\operatorname{ord}\left(\phi^{\prime}-\phi\right)>i+k_{\varphi}
$$

$u_{j}\left(\phi^{\prime}, \varphi\right)=0, \quad 1 \leqslant j \leqslant r\left(\operatorname{pour} l+1 \leqslant j \leqslant r, u_{j}\left(\phi^{\prime}, \varphi\right)=u_{j}(\varphi)=0\right)$.

Il en découle que $\operatorname{ord}\left(\Delta\left(\phi^{\prime}, \varphi\right)\right)=\operatorname{ord}(\Delta(\phi, \varphi))=k_{\varphi}$, donc en particulier $\Delta\left(\phi^{\prime}, \varphi\right) \neq 0$.

Mais sur $V_{0} \subset \mathbb{K}^{n} \times \mathbb{K}^{p}$, on a $\Delta . \mathcal{I} \subset\left(u_{1}, \ldots, u_{r}\right)$.O. Donc $\left(\phi^{\prime}, \varphi\right) \in \mathcal{V}(\mathcal{I})$ et $\operatorname{ord}\left(\phi^{\prime}-\phi\right)>i$, ce que nous désirions.

$$
\underline{2^{i e m e} \text { cas }:} \quad(0, \varphi) \in \mathcal{V}(\mathcal{J}) \text {. }
$$

Il existe alors $k_{\varphi}, a_{\varphi} \in \mathbb{N}$ tels que $\forall \phi \in \mathcal{A}_{\left(\mathbb{K}^{n}, 0\right)}$, on ait :

$$
\begin{aligned}
(\operatorname{ord}(\phi) & \left.>k_{\varphi} \text { et } \operatorname{Min}\left(\operatorname{ord}(\Delta(\phi, \varphi)), \operatorname{Min}_{1 \leqslant j \leqslant k_{0}} \operatorname{ord}\left(f_{j}(\phi, \varphi)\right)\right)>a_{\varphi}+\alpha i\right) \\
& \Longrightarrow\left(\exists \phi^{\prime} \in \mathcal{A}_{\left(\mathbb{K}^{n}, 0\right)} \mid \operatorname{ord}\left(\phi^{\prime}-\phi\right)>i \text { et }\left(\phi^{\prime}, \varphi\right) \in \mathcal{V}(\mathcal{J})\right)
\end{aligned}
$$

Soit alors $\phi \in \mathcal{A}_{\left(\mathbb{K}^{n}, 0\right)}$ tel que $: \operatorname{ord}(\phi)>k_{\varphi}$ et $\operatorname{Min}_{1 \leqslant j \leqslant k_{0}}\left(\operatorname{ord}\left(f_{j}(\phi, \varphi)\right)\right)>$ $2 a_{\varphi}+2 \alpha i$.

Si $\operatorname{ord}(\Delta(\phi, \varphi))>a_{\varphi}+\alpha i$, il existe $\phi^{\prime} \in \mathcal{A}_{\left(\mathbb{K}^{n}, 0\right)}$ avec $\operatorname{ord}\left(\phi^{\prime}-\phi\right)>i$ et $\left(\phi^{\prime}, \varphi\right) \in \mathcal{V}(\mathcal{J}) \subset \mathcal{V}(\mathcal{I})$. Nous avons alors fini. On peut donc supposer que :

$$
\operatorname{ord}(\Delta(\phi, \varphi)) \leqslant a_{\varphi}+\alpha i
$$

Donc en particulier : $\operatorname{ord}(u(\phi, \varphi)) \leqslant a_{\varphi}+\alpha i$. 
On procède alors comme dans le premier cas, en considérant le système d'équations implicites :

$U_{j}(t, z)=u_{j}\left(\phi_{1}(t)+z_{1}, \ldots, \phi_{l}(t)+z_{l}, \phi_{l+1}(t), \ldots, \phi_{n}(t), \varphi(t)\right)=0,1 \leqslant j \leqslant l$.

On a :

$$
U_{j}(t, 0) \in\left(\operatorname{det}\left(\frac{\partial U_{k}}{\partial z_{j}}(t, 0)\right)^{2} \cdot t^{2\left(a_{\varphi}+\alpha i-\operatorname{ord}(u(\phi, \varphi))\right)+1}\right) .
$$

D'où l'existence de $\theta_{1}, \ldots, \theta_{l} \in \mathbb{K}\{t\}$ tels que :

$\operatorname{ord}\left(\theta_{s}\right) \geqslant a_{\varphi}+\alpha i+1+\left(a_{\varphi}+\alpha i-\operatorname{ord}(u(\phi, \varphi))\right) \geqslant a_{\varphi}+\alpha i+1, \quad, 1 \leqslant s \leqslant l$, et $U_{s}\left(t, \theta_{1}(t), \ldots, \theta_{l}(t)\right)=0,1 \leqslant s \leqslant l$.

Posons $\phi^{\prime}=\left(\phi_{1}+\theta_{1}, \ldots, \phi_{l}+\theta_{l}, \phi_{l+1}, \ldots, \phi_{n}\right)$. On a $: \operatorname{ord}\left(\Delta\left(\phi^{\prime}, \varphi\right)\right)$ $=\operatorname{ord}(\Delta(\phi, \varphi)) \leqslant a_{\varphi}+\alpha i$. On conclut alors comme dans le premier cas.

\section{Suite des multiplicités de Nash d'un espace analytique le long d'un arc}

Les notions de suite des mutiplicités de Nash (Resp. suite de Nash des fonctions de Hilbert-Samuel et suite de Nash des diagrammes des exposants initiaux) d'un espace analytique le long d'un arc ont été introduites dans la Définition 1.5. Pour les notations employées, nous prions le lecteur de se reporter à l'introduction.

Le Théorème 1.6 découle directement du résultat suivant. Celui-ci ne surprendra pas les «désingularisateurs». On en trouve trace au moins dans le cas complexe, sous une forme un peu différente, dans [L-J-T] th. 2.13 p.242. Nous donnons ici une preuve valable en réel et complexe, qui procède par réduction au cas hypersurface. Cette preuve est nous semble-t-il plus effective que celle de [L-J-T].

Pour énoncer ce résultat, considérons un germe d'espace analytique $(X, x)$ $\hookrightarrow\left(\mathbb{K}^{n}, 0\right)$, et $\varphi \in \mathcal{A}_{\left(\mathbb{K}^{n}, 0\right)}$ un arc non trivial. Nous notons par $(C, 0)$ le germe de courbe image de $(\mathbb{K}, 0)$ par $\varphi$. Soit $H_{X, C}$ la valeur générique de la fonction de Hilbert-Samuel de $X$ le long de la courbe $C$. C'est donc la valeur commune des $H_{X, \varphi(t)}$ pour $t \neq 0$ assez petit. 
Considérons maintenant le diagramme commutatif suivant :

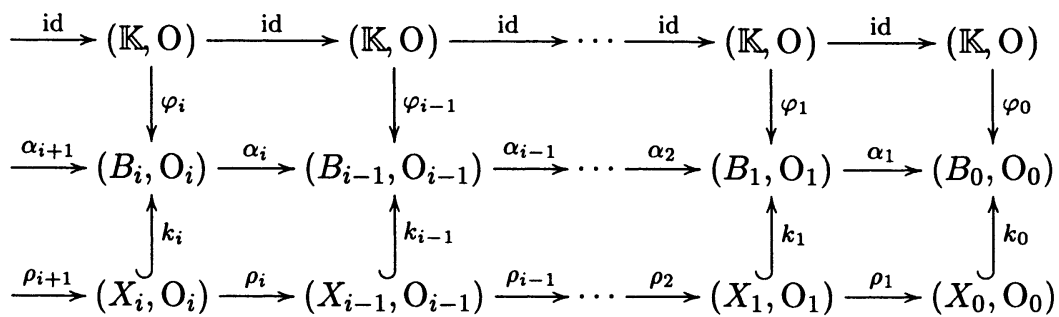

où :

- $\left(B_{0}, O_{0}\right)=\left(\mathbb{K}^{n}, 0\right)$ et $\left(X_{0}, O_{0}\right)=(X, x)$;

- $\alpha_{i}$ est l'éclatement de $B_{i-1}$ de centre $O_{i-1}$;

- $\varphi_{i}$ relève $\varphi_{i-1}$ à travers $\alpha_{i}, O_{i}=\varphi_{i}(0)$;

- $X_{i}$ est le transformé strict de $X_{i-1}$ par $\alpha_{i}, \rho_{i}=\alpha_{i} / X_{i}$ et les $k_{i}$ sont les plongements induits.

ThÉORÈme 4.1. - Soit $H_{i}=H_{X_{i}, O_{\imath}}$ la fonction de Hilbert-Samuel du germe $\left(X_{i}, O_{i}\right)$. La suite $H_{i}$ est décroissante et il existe $i_{0}$ tel que $H_{i}=$ $H_{X, C}, \forall i \geqslant i_{0}$. En particulier la suite des multiplicités $m_{i}$ successives est décroissante et se stabilise à la multiplicité générique de $X$ le long de $C$. Par suite, pour $\varphi \in \mathcal{R}_{(X, 0)}$, les germes $\left(X_{i}, O_{i}\right)$ sont lisses pour $i \geqslant i_{0}$.

Avant de prouver 4.1, notons que celui-ci entraine 1.6. En effet, partant d'un germe $(X, x)$ et d'un $\operatorname{arc} \varphi \in \mathcal{A}_{\left(\mathbb{K}^{n}, 0\right)}$, on applique 4.1 à $Z_{0}=(\mathbb{K}, 0) \times(X, x)$ et à l'arc $\Gamma_{0}$. Ceci fournit le point 2) de 1.6. Pour le point 1 ), il suffit de remarquer que la multiplicité de $X$ en $y$ est la même que celle de $Z_{0}$ en $(t, y)$.

Preuve de 4.1. - Démontrons d'abord le cas où $(X, x)$ est un germe d'hypersurface à l'origine de $\mathbb{K}^{n}$. Dans ce cas, la fonction d'Hilbert-Samuel est entièrement déterminée par la multiplicité. Il s'agit donc de voir que la suite des multiplicités successives $m_{i}$ des $\left(X_{i}, O_{i}\right)$ tend vers $m_{0}^{\prime}$, où $m_{0}^{\prime}$ est la multiplicité générique de $X$ le long de $(C, 0)$. Soit $m_{i}^{\prime}$ la multiplicité générique de $\left(X_{i}, O_{i}\right)$ le long de $\left(C_{i}, O_{i}\right)$, où $\left(C_{i}, O_{i}\right)=\operatorname{Im} \varphi_{i}$. On a :

i) $m_{i}^{\prime} \leqslant m_{i}$ par semi-continuité supérieure de la multiplicité.

ii) $m_{i}^{\prime}=m_{0}^{\prime}$ car $\alpha_{i}$ est un isomorphisme de $B_{i}-\alpha_{i}^{-1}\left(O_{i-1}\right) \rightarrow B_{i}-$ $\left\{O_{i-1}\right\}$. 
Donc $m_{0}^{\prime} \leqslant m_{i}$ pour tout $i \in \mathbb{N}$.

D'autre part, soit $f \in \mathbb{K}\left\{x_{1}, \ldots, x_{n}\right\}=\mathcal{O}_{n}$, un germe d'équation pour notre hypersurface.

$$
f(x+y)=\sum_{\alpha \in \mathbb{N}^{n}} \frac{1}{\alpha !} \frac{\partial^{|\alpha|} f(x)}{\partial x^{\alpha}} y^{\alpha} .
$$

Considérons, pour $k \in \mathbb{N}, J_{k}$ l'idéal de $\mathcal{O}_{n}$ engendré par les dérivées partielles $\frac{\partial^{|\alpha|} f(x)}{\partial x^{\alpha}}$ avec $|\alpha| \leqslant k$. Alors :

$$
m_{0}^{\prime}=\operatorname{Min}\left\{k \in \mathbb{N} / \varphi^{*} J_{k} \neq 0\right\} .
$$

Posons $D=\operatorname{ord} \varphi^{*}\left(J_{m_{0}^{\prime}}\right)<+\infty$. On a alors : $\forall i \geqslant D, m_{i}=m_{0}^{\prime}$.

En effet, si cela n'était pas le cas, on aurait, compte-tenu de la décroissance de la suite des multiplicités (cas particulier du théorème de Bennett $[\mathrm{B}]$ ) :

$$
\text { (*) } \quad m_{i}>m_{0}^{\prime} \text { pour } 0 \leqslant i \leqslant D .
$$

Ceci est absurde, comme il va résulter du lemme suivant :

Lemme 4.2. - Soit $i \in \mathbb{N}$, notons $f_{i} \in \mathcal{O}_{n}$, la i.ème transformée stricte de $f$, et pour $k \in \mathbb{N}$, considérons $J_{i, k}$ l'idéal de $\mathcal{O}_{n}$ engendré par les $\frac{\partial^{|\alpha|} f_{2}(x)}{\partial x^{\alpha}}$ avec $|\alpha| \leqslant k$. Alors si $k<m_{i}$, on $a$ :

$$
\operatorname{ord}\left(\varphi_{i}^{*} J_{i, k}\right) \geqslant 1+\operatorname{ord}\left(\varphi_{i+1}^{*} J_{i+1, k}\right) .
$$

Appliquant successivement ceci, pour $i$ variant de 0 à $D$, avec $k=m_{0}^{\prime}$, on aurait alors par $(*)$ :

$$
\operatorname{ord}\left(\varphi^{*} J_{m_{0}^{\prime}}\right) \geqslant(D+1)+\operatorname{ord}\left(\varphi_{D+1}^{*} J_{D+1, m_{0}^{\prime}}\right) .
$$

Ce qui est absurde car ord $\left(\varphi^{*} J_{m_{0}^{\prime}}\right)=D$. Il nous reste donc à prouver (4.2).

Preuve de 4.2.- On peut trouver un système de coordonnées et un isomorphisme local :

$$
u_{i+1}:\left(B_{i+1}, O_{i+1}\right) \rightarrow\left(\mathbb{K}^{n}, 0\right)
$$

tel que le germe d'éclatement

$$
\begin{aligned}
\alpha_{i+1}:\left(B_{i+1}, O_{i+1}\right) \simeq & \left(\mathbb{K}^{n}, 0\right) \rightarrow\left(B_{i}, O_{i}\right) \simeq\left(\mathbb{K}^{n}, 0\right) \\
& -20-
\end{aligned}
$$


soit représenté par :

$$
\left(x_{1}, x_{2}, \ldots, x_{n}\right) \rightarrow\left(x_{1}, x_{1}\left(a_{2}+x_{2}\right), \ldots, x_{1}\left(a_{n}+x_{n}\right)\right), a_{i} \in \mathbb{K}, i \geqslant 2 .
$$

On a alors :

$$
f_{i}\left(x_{1}, x_{1}\left(a_{2}+x_{2}\right), \ldots, x_{1}\left(a_{n}+x_{n}\right)\right)=x_{1}^{m_{\imath}} u\left(x_{1}, \ldots, x_{n}\right) f_{i+1}\left(x_{1}, \ldots, x_{n}\right)
$$

où $u$ est un inversible de $\mathcal{O}_{n}$.

On vérifie par récurrence sur $k \leqslant m_{i}$, que pour $|\alpha| \leqslant k$, on a :

$$
\frac{\partial^{|\alpha|} f_{i}}{\partial x^{\alpha}}\left(x_{1}, x_{1}\left(a_{2}+x_{2}\right), \ldots, x_{1}\left(a_{n}+x_{n}\right)\right) \in x_{1}^{m_{\imath}-k} J_{i+1, k} .
$$

Composant ceci avec $\varphi_{i+1}$ (écrite dans le système de coordonnées), on obtient :

$\operatorname{ord}\left(\varphi_{i}^{*} J_{i, k}\right) \geqslant\left(m_{i}-k\right)$ ord $\left(\varphi_{i}\right)+\operatorname{ord}\left(\varphi_{i+1}^{*} J_{i+1, k}\right) \geqslant 1+\operatorname{ord}\left(\varphi_{i+1}^{*} J_{i+1, k}\right)$

Ceci achève la preuve du cas où $(X, 0)$ est un germe d'hypersurface. Notons dans ce cas que pour $i \geqslant D, X_{i}$ est équimultiple le long de $C_{i}=\operatorname{Im} \varphi_{i}$.

Prouvons à présent le cas général. Soit $H_{X_{\imath}, C_{\imath}}$ la valeur générique de la fonction de Hilbert-Samuel de $X_{i}$ le long de $C_{i}=\operatorname{Im} \varphi_{i}$.

Chaque $\alpha_{i}$ induisant un isomorphisme de $B_{i}-\alpha_{i}^{-1}\left(O_{i}\right)$ sur $B_{i-1}-\left\{O_{-1}\right\}$, on a :

i) la suite $H_{X_{\imath}, C_{\imath}}$ est constante et égale à $H_{X, C}$;

ii) $H_{i+1}=H_{X_{\imath+1}, O_{\imath+1}} \leqslant H_{i}=H_{X_{\imath}, O_{\imath}}$ par le théorème de Bennett [B] ;

iii) par semi-continuité de la fonction de Hilbert-Samuel (cf. [L-J-T]) :

$$
H_{X, C}=H_{X, C_{\imath}} \leqslant H_{i}=H_{X_{\imath}, O_{\imath}} .
$$

Par conséquent, montrer qu'il existe $i_{0}$ tel que, $\forall i \geqslant i_{0}$ on ait $H_{i}=H_{X, C}$, c'est montrer qu'il existe $i_{0}$ tel que $\forall i \geqslant i_{0}$ on ait $H_{i}=H_{X_{2}, C_{2}}$. Ce qui signifie qu'il s'agit de voir que $\left(C_{i}, O_{i}\right) \subset\left(S_{i}, O_{i}\right)$ où $S_{i}$ est la strate de Hilbert-Samuel de $X_{i}$ passant par $O_{i}$ i.e. :

$$
S_{i}=\left\{x \in X_{i} \mid H_{X_{\imath}, x}=H_{X_{\imath}, O_{\imath}}\right\} .
$$

Plongeons $\left(X_{i}, O_{i}\right)$ dans $\left(\mathbb{K}^{n}, 0\right)$ et choisissons un système de coordonnées quelconques, $y_{1}, \ldots, y_{n}$, à l'origine de $\mathbb{K}^{n}$. Écrivons :

$$
\mathcal{O}_{X_{\imath}, O_{\imath}}=\frac{\mathbb{K}\{y\}}{\mathfrak{a}_{i}}
$$


Si $f_{i, 1}, \ldots, f_{i, p_{\imath}}$ désigne la base standard distinguée de $\mathfrak{a}_{\imath}$ dans ce système de coordonnées, on a :

$$
S_{i}=\cap_{j=1}^{p_{2}} S_{i, j} .
$$

Avec $S_{i, j}=\left\{x \in \mathbb{K}^{n} \mid m_{x}\left(f_{i, j}\right)=m_{O_{\imath}}\left(f_{i, j}\right)\right\}$, où $m_{x}$ désigne la multiplicité ou l'ordre au point $x$ (cf. [B-M2] Th. 5.3.1 p.821).

Notre problème revient donc à voir qu'il existe $i_{0}$, tel que pour tout $i \geqslant i_{0}$, on puisse trouver un système de coordonnées à l'origine de $\left(\mathbb{K}^{n}, 0\right)$ tel que chaque élément de la base standard distinguée $f_{i, j}$, avec $1 \leqslant j \leqslant p_{i}$, relative à ce système de coordonnées, soit équimultiple le long de $C_{i}$. Nous allons prouver cette affirmation. Puisque pour tout $i$ :

$$
H_{i+1} \leqslant H_{i},
$$

par le théorème de stabilisation 5.2.2 p. 820 de [B-M2], on obtient l'existence d'un $i_{0}$ tel que $\forall i \geqslant i_{0}$ :

$$
H_{i}=H_{i_{0}} .
$$

Fixons un tel entier $i_{0}$, plongeons $\left(X_{i_{0}}, O_{i_{0}}\right)$ dans $\left(\mathbb{K}^{n}, 0\right)$, et choisissons un système de coordonnées (à l'origine de $\left.\mathbb{K}^{n}\right)(w, z)$ avec $w=\left(w_{1}, \ldots, w_{n-r}\right)$, $z=\left(z_{1}, \ldots, z_{r}\right)$ satisfaisant les conclusions du lemme 7.2 p.828 de [B-M2] (des variables essentielles dans la terminologie de [B-M2]).

Considérons maintenant $f_{i_{0}, 1}, \ldots, f_{i_{0}, p_{\imath_{0}}}$, la base standard distinguée de $\mathfrak{a}_{i_{0}}$ relativement à ce système de coordonnées, où $\mathcal{O}_{X_{\imath_{0}}, O_{v_{0}}}=\frac{\mathbb{K}\{w, z\}}{\mathfrak{a}_{z_{0}}}$. D'après le théorème 7.3 p. 828 de [B-M2], il existe un système de coordonnées $\left(w^{\prime}, z^{\prime}\right)$ dans un voisinage de $O_{i_{0}+1}=0$ tel que :

- si $\mathfrak{a}_{i_{0}+1} \subset \mathbb{K}\left\{w^{\prime}, z^{\prime}\right\}$ désigne le transformé strict de $\mathfrak{a}_{i_{0}}$ (donc $\left.\mathcal{O}_{X_{i_{0}+1}, O_{i_{0}+1}} \simeq \mathbb{K}\left\{w^{\prime}, z^{\prime}\right\} / \mathfrak{a}_{i_{0}+1}\right)$, la base standard distinguée de $\mathfrak{a}_{i_{0}+1}$ est constituée par les transformées strictes $f_{i_{0}+1, j} \operatorname{des} f_{i_{0}, j}, 1 \leqslant j \leqslant p_{i_{0}}$.

- le système de coordonnées $\left(w^{\prime}, z^{\prime}\right)$ satisfait les conclusions du lemme 7.2 p.828 de [B-M2].

On peut donc itérer ce qui précède. D'après le cas hypersurface, au bout d'un nombre fini d'étapes $\mathrm{D}$, les $f_{i_{0}+k, j}, 1 \leqslant j \leqslant p_{i_{0}}, k \geqslant D$ deviennent équimultiples le long de $C_{i_{0}+k}$. Le théorème en résulte comme nous l'avons vu plus haut.

Remarque 4.3. - Soit $\varphi \in \mathcal{R}_{(X, 0)}$. Supposons que $(X, 0)$ soit réduit et équidimensionnel de dimension $d$. Soit $I \subset \mathcal{O}_{n}$ tel que $\mathcal{O}_{X, 0}=\mathcal{O}_{n} / I$. Si $J$ désigne l'idéal engendré par $I$ et tous les jacobiens d'ordre $n-d$ des $n-d$ upplets d'éléments de $I$, et si $D=\operatorname{ord}\left(\varphi^{*}(J)\right)$, on a $\left(X_{i}, O_{i}\right)$ lisse pour $i \geqslant D$. Il suffit pour le constater de reprendre le calcul du Lemme 4.2. 
Le Théorème 4.1 et la Remarque 4.3 nous permettent de décrire une base de voisinage d'un élément de $\mathcal{R}_{(X, 0)}$. Pour cela, plongeons $(X, 0)$ dans $\left(\mathbb{K}^{n}, 0\right)$ et notons par d la dimension en 0 de $(X, 0)$. Soit $z=\left(z^{\prime}, z^{\prime \prime}\right)$, $z^{\prime}=\left(z_{1}, \ldots, z_{d}\right), z^{\prime \prime}=\left(z_{d+1}, \ldots, z_{n}\right)$ un système de coordonnées à l'origine de $\mathbb{K}^{n}$. Pour $\varphi \in \mathcal{R}_{(X, 0)}$, notons comme prédemment :

$$
\varphi(t)=\left(\varphi_{1}(t), \ldots, \varphi_{n}(t)\right)=\sum_{k=1}^{+\infty} A_{k} \cdot t^{k} .
$$

Soit $\xi \in B_{\mathbb{K}^{n}, i}(\varphi)$. Posons : $\xi(t)=\sum_{k=0}^{i} A_{k} \cdot t^{k}+t^{i} r_{i}(\xi)(t)$, où $r_{i}(\xi) \in$ $\mathcal{A}_{\left(\mathbb{K}^{n}, 0\right)}$. Notons $: r_{i}(\xi)=\left(r_{i}^{\prime}(\xi), r^{\prime \prime}(\xi)\right), r_{i}^{\prime}(\xi)=\left(r_{i, 1}(\xi), \ldots, r_{i, d}(\xi)\right) \in \mathcal{A}_{\left(\mathbb{K}^{d}, 0\right)}$, $r^{\prime \prime}{ }_{i}(\xi)=\left(r_{i, d+1}(\xi), \ldots, r_{i, n}(\xi)\right) \in \mathcal{A}_{\left(\mathbb{K}^{n-d}, 0\right)}$.

Corollaire 4.4. - Soit $\varphi(t)=\sum_{k=1}^{+\infty} A_{k} . t^{k}, \varphi \in \mathcal{R}_{(X, 0)}$. Il existe $i_{0} \in \mathbb{N}$, tel que $\forall i \geqslant i_{0}$, après une éventuelle permutation des coordonnées dans $\mathbb{K}^{n}$, il existe des germes de fonctions analytiques nulles à l'origine dans $\mathbb{K}^{d+1}, u_{i, j}\left(z^{\prime}, t\right), d+1 \leqslant j \leqslant n$, tels que pour $\xi \in B_{\left(\mathbb{K}^{n}, i\right)}(\varphi)$ on ait :

$$
\xi \in B_{X, i}(\varphi) \Longleftrightarrow r{ }_{i}(\xi)(t)=\left(u_{i, d+1}\left(r_{i}^{\prime}(\xi)(t), t\right), \ldots, u_{n}\left(r_{i}^{\prime}(\xi)(t), t\right)\right)
$$

Preuve - - Conservant les notations qui suivent $\left(D_{2}\right)$, on a $\xi \in B_{X, i}(\varphi)$ si et seulement si l'arc $t \rightarrow\left(t, r_{i}(\xi)(t)\right) \in \mathcal{A}_{\left(Z_{i}^{\prime}, 0\right)}$. Il ne nous reste plus qu'à décrire $\mathcal{A}_{\left(Z_{2}^{\prime}, 0\right)}$. Fixons $i_{0}$ de telle sorte que pour $i \geqslant i_{0},\left(Z_{i}^{\prime}, 0\right)$ soit lisse. Comme $\operatorname{dim}_{0}\left(Z_{i}^{\prime}, 0\right)=d+1$, celui-ci peut être défini par :

$$
\left(Z_{i}^{\prime}, 0\right)=\left\{(t, z) \in(\mathbb{K}, 0) \times\left(\mathbb{K}^{n}, 0\right) \mid f_{d+1}(t, z)=\ldots=f_{n}(t, z)=0\right\},
$$

où le rang en 0 de la matrice jacobienne des $f_{j}, d+1 \leqslant j \leqslant n$, est $n-d$. Pour conclure, il nous suffit de voir, par le théorème des fonctions implicites ordinaires, qu'il existe $i_{d+1}, \ldots, i_{n} \in\{1, \ldots, n\}$ tels que :

$$
\operatorname{det}\left(\frac{\partial f_{k}}{\partial z_{i_{l}}}\right)_{d+1 \leqslant k, l \leqslant n}(0) \neq 0 .
$$

Ceci est nécessairement satisfait. En effet, si cela n'était pas le cas, puisque le rang de la matrice jacobienne des $f_{j}$ est tout de même $n-d$, cela signifierait que l'on peut définir (après une éventuelle permutation des $\left.z_{j}\right)\left(Z_{i}^{\prime}, 0\right)$ comme : 


$$
\begin{aligned}
\left(Z_{i}^{\prime}, 0\right) & =\left\{(t, z) \in(\mathbb{K}, 0) \times\left(\mathbb{K}^{n}, 0\right) / t-u_{1}\left(z_{1}, \ldots, z_{d+1}\right)\right. \\
& =z_{d+2}-u_{2}\left(z_{1}, \ldots, z_{d+1}\right) \\
& \left.=\ldots=z_{n}-u_{n-d}\left(z_{1}, \ldots, z_{d+1}\right)=0\right\}
\end{aligned}
$$

avec $u_{j}(0)=0,1 \leqslant j \leqslant n-d$ et $\partial u_{1} / \partial z_{1}(0)=\partial u_{1} / \partial z_{2}(0)=\ldots=$ $\partial u_{1} / \partial z_{d+1}(0)=0$.

Mais ceci est absurde puisque l'arc $t \rightarrow\left(t, r_{i}(\varphi)(t)\right)$ est à valeurs dans $\left(Z_{i}^{\prime}, 0\right)$. Or celui-ci ne peut satisfaire l'équation :

$$
t-u_{1}\left(r_{i, 1}(\varphi)(t), \ldots, r_{i, d+1}(\varphi)(t)\right)=0,
$$

$\operatorname{car} \operatorname{ord}\left(u_{1}\left(r_{i}(\varphi)\right) \geqslant 2\right.$.

Remarque 4.5 . - 1) Soit $\varphi \in \mathcal{R}_{(X, 0)}$. Fixons $i_{0}$ satisfaisant les conditions de 4.4. Soit $i \geqslant i_{0}$, alors l'application $\Theta_{i}$ :

$$
\begin{gathered}
B_{X, i}(\varphi) \rightarrow \mathcal{A}_{\left(\mathbb{K}^{d}, 0\right)} \\
\xi \rightarrow r_{i}^{\prime}(\xi)
\end{gathered}
$$

est un "isomorphisme analytique" puisque sa réciproque $\Theta_{i}^{-1}$ est donnée par :

$$
\begin{gathered}
\mathcal{A}_{\left(\mathbb{K}^{d}, 0\right)} \rightarrow B_{X, i}(\varphi) \\
\alpha \rightarrow \Theta_{i}^{-1}(\alpha)
\end{gathered}
$$

où $\Theta_{i}^{-1}(\alpha)(t)=\left(\alpha_{1}(t), \ldots, \alpha_{d}(t), u_{i, d+1}(\alpha(t), t), \ldots, u_{i, n}(\alpha(t), t)\right)$. On peut d'autre part considérer que la notion naturelle de morphisme analytique entre $\mathcal{A}_{(X, 0)}$ et $\mathcal{A}_{(Y, 0)}$ est celle des morphismes induits par les applications analytiques :

$$
\begin{gathered}
(\mathbb{K}, 0) \times(X, 0) \rightarrow(\mathbb{K}, 0) \times(Y, 0) \\
(t, x) \rightarrow(t, a(t, x)) .
\end{gathered}
$$

Pour ces raisons, il nous semble justifié de dire que de ce point de vue $\mathcal{R}_{(X, x)}$ est l'ensemble des points réguliers de $\mathcal{A}_{(X, x)}$.

2) $\mathrm{Si}(X, 0)$ est réduit et équidimensionnel, l'entier $i_{0}$ est précisé par 4.3.

3) Spécifier la dépendance des $u_{i, j}\left(z^{\prime}, t\right)$ par rapport aux $A_{k}, k \leqslant i$, est la notion de stabilité de [D-L1]. Ceci découlera du Théorème 4.10. L'entier $i_{0}$ de 4.3 est plus précis que celui fourni par la preuve du Lemme 4.1 de [D-L1]. Il est utile de préciser cet entier $i_{0}$ si on cherche à obtenir des informations précises sur la série de Poincaré considérée dans [D-L1]. 
En général $\mathcal{R}_{(X, 0)}$ n'est pas dense dans $\mathcal{A}_{(X, 0)}$ comme le montre l'exemple suivant :

Exemple 4.6 . - Considérons le germe en 0 du parapluie de Whitney :

$$
(X, 0)=\left\{\left(z_{1}, z_{2}, z_{3}\right) \in\left(\mathbb{C}^{3}, 0\right) \mid z_{1}^{2}-z_{2} z_{3}^{2}=0\right\}
$$

$(\operatorname{Sing}(X), 0)=\left\{\left(z_{1}, z_{2}, z_{3}\right) \in\left(\mathbb{C}^{3}, 0\right) \mid z_{1}=z_{3}=0\right\}$. Soit l'arc $\varphi, t \rightarrow(0, t, 0)$. Il est facile de vérifier que $\forall i \geqslant 1, B_{X, i}(\varphi) \subset \mathcal{A}_{(\operatorname{Sing}(X), 0)}$. Par conséquent $B_{X, i}(\varphi) \cap \mathcal{R}_{(X, 0)}=\varnothing$.

Cependant ce genre de pathologie peut toujours être évité par un changement de paramétrisation. Plus précisément on a :

Lemme 4.7. - Soient $(X, 0)$ un germe d'espace analytique complexe réduit et $\varphi \in \mathcal{A}_{(X, 0)}$. Alors il existe un entier $p \in \mathbb{N}^{*}$ tel que, désignant par $\theta$ l'arc $t \rightarrow \varphi\left(t^{p}\right)$, on ait, $\forall(Y, 0)$ sous-ensemble analytique propre de $(X, 0)$ :

$$
\forall i \in \mathbb{N}, B_{X, i}(\theta) \cap\left(\mathcal{A}_{(X, 0)}-\mathcal{A}_{(Y, 0)}\right) \neq \varnothing .
$$

Preuve. - Fixons un représentant de $(X, 0)$ dans un voisinage de 0 assez petit. Soit alors $\Pi: Z \rightarrow X$, avec $Z$ lisse dénombrable à l'infini, $\Pi$ propre et surjectif (un tel $\Pi$ existe en vertu des résultats sur la désingularisation). Soit maintenant $C$ la courbe image de $\varphi$. Posons $A^{\prime}=\Pi^{-1}(C)$. Considérons la décomposition de $A^{\prime}$ en composantes irréductibles $A^{\prime}=\cup_{\alpha \in \Lambda} A_{\alpha}^{\prime}(\Lambda$ est dénombrable). Pour chaque $\alpha \in \Lambda$, puisque $\Pi$ est propre, $\Pi\left(A_{\alpha}^{\prime}\right)$ est un sous-ensemble analytique irréductible inclus dans $C$. C'est donc un point ou $C$ tout entier. Comme $\Lambda$ est dénombrable, il existe $\alpha$ tel que $\Pi\left(A_{\alpha}^{\prime}\right)=C$. Fixons un tel $\alpha$, disons $\alpha=1$. $\Pi^{-1}(0) \cap A_{1}^{\prime}$ est un sous-ensemble analytique de $A_{1}^{\prime}$ tel que pour tout $p \in A_{1}^{\prime}, \operatorname{dim}_{p} \Pi^{-1}(0) \cap A_{1}^{\prime}<\operatorname{dim}_{p} A_{1}^{\prime}$. Soit $p \in \Pi^{-1}(0) \cap A_{1}^{\prime}$, considérons un arc $\rho:(\mathbb{C}, 0) \rightarrow\left(A_{1}^{\prime}, p\right)$ tel que $\rho \in \mathcal{A}_{\left(A_{1}^{\prime}, p\right)}-\mathcal{A}_{\left(\Pi^{-1}(0) \cap A_{1}^{\prime}, p\right)}$. On peut supposer que $\rho$ définit la normalisation de son image $\left(C_{0}, p\right)$. On a alors un diagramme commutatif induit par $\Pi$ :

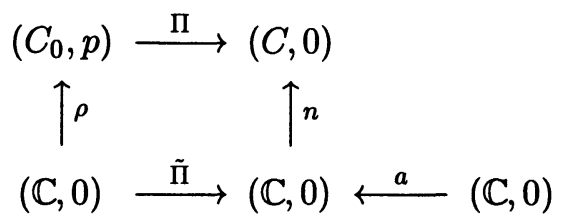

où $\varphi=n \circ a, n$ est est la normalisation de $(C, 0), \tilde{\Pi}$ le relèvement de $\Pi$ à la normalisation. Par un choix convenable de coordonnée dans le $(\mathbb{C}, 0)$ source de $\tilde{\Pi}$, on peut écrire $\tilde{\Pi}(t)=t^{p}$. Ainsi $\Pi(\rho(t))=n\left(t^{p}\right)$. Écrivons maintenant 
$a(t)=t^{k} u(t), u(0) \neq 0$. Puis soit $\alpha \in \mathcal{O}_{1}$ tel que $\alpha(t)^{p}=u\left(t^{p}\right)$. On a :

$$
\varphi\left(t^{p}\right)=n\left(t^{k p} u\left(t^{p}\right)\right)=n\left(\left(t^{k} \alpha(t)\right)^{p}\right)=\Pi(\rho(t \alpha(t))) .
$$

Si $\tilde{\rho}$ désigne l'arc $t \rightarrow \rho(t \alpha(t)) \in \mathcal{A}_{(Z, p)}$, on a donc $\Pi(\tilde{\rho}(t))=\varphi\left(t^{p}\right)=\theta(t)$. Soit maintenant $(Y, 0)$ un sous-ensemble analytique propre de $(X, 0)$. Notons $Y^{\prime}=\Pi^{-1}(Y),\left(Y^{\prime}, p\right)$ est un sous-ensemble analytique propre de $(Z, p)$. Soit $i \in \mathbb{N}$, il existe en vertu du lemme 4.9 ci-dessous $\tilde{\xi} \in B_{Z, i}(\tilde{\rho})$ tel que $\tilde{\xi} \notin \mathcal{A}_{\left(Y^{\prime}, p\right)}$. Par suite l'arc $\xi=\Pi(\tilde{\xi}) \in B_{X, i}(\theta)$ et $\notin \mathcal{A}_{(Y, 0)}$.

Remarque 4.8 . - Le Lemme 4.7 ne sera pas utilisé par la suite. C'est le seul endroit où nous utilisons l'existence de désingularisation. Il a été motivé par une affirmation dans l'article de J. Becker référencé dans [I5].

Le lemme 4.9 est une version effective du second point du Lemme 1.4 de [T4]. Cette effectivité sera utilisée à la section suivante.

LEMME 4.9.-

Soient $\varphi \in \mathcal{A}_{\left(\mathbb{K}^{n}, 0\right)}$ et $i \in \mathbb{N}$. Pour $f \in \mathbb{K}\left[\left[X_{1}, \ldots, X_{n}\right]\right], f \neq 0$ on $a$ :

$$
m_{0}(f) \leqslant \operatorname{Min}_{\theta \in B_{\mathbb{K}^{n}, 2}(\varphi)} \operatorname{ord}(f \circ \theta) \leqslant m_{0}(f)(i+1)
$$

où $m_{0}(f)$ désigne la multiplicité ou l'ordre de $f$.

Preuve. - Soient $\varphi \in \mathcal{A}_{\left(\mathbb{K}^{n}, 0\right)}$ et $i \in \mathbb{N}$. Pour prouver 4.9, on peut supposer $\operatorname{ord}(\varphi) \leqslant i$. En effet sinon, soit $\left(a_{1}, \ldots, a_{n}\right)$ ne figurant pas dans les zéros de la forme initiale de $f$. Alors l'arc $t \rightarrow \theta(t)=\left(a_{1} t^{i+1}, \ldots, a_{n} t^{i+1}\right)$ est dans $B_{\mathbb{K}^{n}, i}(\varphi)$ et $\operatorname{ord}(f \circ \theta)=m_{0}(f)(i+1)$. Nous supposerons donc $\operatorname{ord}(\varphi) \leqslant$ $i$. On peut par ailleurs supposer que $f \in \mathcal{O}_{n}$. Soit $\left(Z_{0}, 0\right)=(\mathbb{K}, 0) \times(X, x)$, où $(X, x)$ est le germe défini par $f$. Posons : $f_{0} \in \mathbb{K}\{t, X\}, f_{0}=f$. Puis soit $f_{j}(t, x) \in \mathcal{O}_{n+1}=\mathbb{K}\{t, X\}$, la transformée stricte de $f_{j-1}$ par $\Pi_{j}^{\prime}$ (les notations sont celles de $\left.\left(D_{2}\right)\right)$. Un calcul élémentaire en coordonnées locales montre que :

$$
t^{m_{0}\left(f_{0}\right)+m_{0}\left(f_{1}\right)+\ldots+m_{0}\left(f_{\imath-1}\right)} f_{i}(t, X)=f\left(\sum_{k=1}^{i} A_{k} t^{k}+t^{i} X\right)
$$

pour $(t, X)$ voisin de $(0,0)$.

Soit alors un point $\left(1, a_{1}, \ldots, a_{n}\right)$ qui n'est pas dans les zéros de la forme initiale de $f_{i}$. On a alors :

$$
t^{m_{0}\left(f_{0}\right)+m_{0}\left(f_{1}\right)+\ldots+m_{0}\left(f_{2-1}\right)} f_{i}\left(t, a_{1} t, \ldots, a_{n} t\right)=f\left(\sum_{k=1}^{i} A_{k} t^{k}+t^{i+1} A\right)
$$


avec $A=\left(a_{1}, \ldots, a_{n}\right)$. D'où :

$$
\operatorname{ord}\left(f\left(\sum_{k=1}^{i} A_{k} t^{k}+t^{i+1} A\right)\right)=m_{0}\left(f_{0}\right)+m_{0}\left(f_{1}\right)+\ldots+m_{0}\left(f_{i-1}\right)+m_{0}\left(f_{i}\right) .
$$

Or c'est un cas extrêmement simple (vérifiable à la «main») du théorème de Benett $[\mathrm{B}]$ de voir que :

$$
m_{0}\left(f_{i}\right) \leqslant m_{0}\left(f_{i-1}\right) \leqslant \ldots \leqslant m_{0}\left(f_{0}\right) .
$$

Par suite $\operatorname{ord}\left(f\left(\sum_{k=1}^{i} A_{k} t^{k}+t^{i+1} A\right)\right) \leqslant m_{0}(f)(i+1)$.

Nous allons à présent prouver le théorème de semi-continuité 1.7 .

Preuve de 1.7. - Considérons une sous-variété algébrique affine irréductible $V \subset \mathbb{K}^{l}$. Notons $\mathbb{K}[V]$ la $\mathbb{K}$-algèbre de type fini qui la définit. Soient $F_{1}(t, X), \ldots$, $F_{s}(t, X)$ des éléments de $\mathbb{K}[V][[t, X]]$. Posons :

$$
F_{j}(t, x)=\sum_{\alpha_{1} \in \mathbb{N}, \alpha \in \mathbb{N}^{n}} a_{\alpha_{1}, \alpha}^{j} t^{\alpha_{1}} X^{\alpha}, a_{\alpha_{1}, \alpha}^{j} \in \mathbb{K}[V] .
$$

Soient $R=\sum_{j=1}^{s} \mathbb{K}[V][[t, X]] F_{j}(t, X)$ et $N(R)$ le diagramme des exposants initiaux de $R$. Pour chaque $v \in V$, il y a un morphisme «d'évaluation des coefficients en $v »$ :

$$
\begin{array}{r}
\mathbb{K}[V][[t, X]] \longrightarrow \mathbb{K}[[t, X]] \\
F(t, X) \longrightarrow F(v, t, X)
\end{array}
$$

où si $F(t, x)=\sum_{\alpha_{1} \in \mathbb{N}, \alpha \in \mathbb{N}^{n}} a_{\alpha_{1}, \alpha} t^{\alpha_{1}} X^{\alpha}, a_{\alpha_{1}, \alpha} \in \mathbb{K}[V]$,

$$
F(v, t, x)=\sum_{\alpha_{1} \in \mathbb{N}, \alpha \in \mathbb{N}^{n}} a_{\alpha_{1}, \alpha}(v) t^{\alpha_{1}} X^{\alpha}, a_{\alpha_{1}, \alpha}(v) \in \mathbb{K} .
$$

On notera par $R_{v}$ l'idéal de $\mathbb{K}[[t, X]]$ engendré par les $F_{j}(v, t, X)$ et par $N\left(R_{v}\right)$ son diagramme des exposants initiaux. On a alors:

Lemme 4.10. - Sous les notations précédentes:

1) $\forall v \in V, N(R) \leqslant N\left(R_{v}\right)$;

2) Il existe $V^{\prime}$ sous-variété algèbrique propre de $V$ telle que :

- $\forall v \in V-V^{\prime}, N(R)=N\left(R_{v}\right) ;$

- pour tout sommet $\beta$ de $N(R)$, il existe $G \in R$ tel que $\nu(G)=\beta$ et $\forall v \in V-V^{\prime}, \nu(G(v, t, X))=\nu(G)=\beta$. 
Preuve. - Il suffit de répéter dans nos notations la preuve des lemmes 7.1 et 7.2 de E. Bierstone et P.D. Milman dans [B-M1].

Lemme 4.11. - Soient $i \in \mathbb{N}^{*}$ et $V$ une sous-variété algébrique irréductible de $\mathcal{A}_{\left(\mathbb{K}^{n}, 0\right)}^{i}=\mathbb{K}^{n i}$, il existe une sous-variété propre $V^{\prime}$ de $V$ telle que :

1) $\forall \varphi^{i}, \theta^{i} \in V-V^{\prime}, \mathcal{H}_{X, \varphi^{2}}^{i}=\mathcal{H}_{X, \theta^{2}}^{i}$;

2) $\forall \varphi^{i}, \theta^{i} \in V-V^{\prime}, \mathcal{N}_{X, \varphi^{2}}^{i}=\mathcal{N}_{X, \theta^{2}}^{i}$.

Preuve. - Nous tentons d'abord d'alléger les notations. Soit $\varphi^{i}$ un élément de $\mathcal{A}_{\left(\mathbb{K}^{n}, 0\right)}^{i}$, c'est donc un morphisme de $\mathbb{K}$-algèbres locales de $\mathbb{K}\left\{X_{1}\right.$, $\left.\ldots, X_{n}\right\} \rightarrow \mathbb{K}\{t\} /(t)^{i+1}$. La donnée de $\varphi^{i}$ est donc équivalente à la donnée d'un élément :

$$
\varphi^{i}=\sum_{k=1}^{i} A_{k} t^{k}, \text { avec } A_{k} \in \mathbb{K}^{n} .
$$

Les notations étant celles de $\left(D_{1}\right)$ et $\left(D_{2}\right)$, nous noterons par $H_{A^{j}}=$ $H_{A_{1}, \ldots, A_{j}}, A^{j}=\left(A_{1}, \ldots, A_{j}\right)$, la fonction de Hilbert-Samuel de $\left(Z_{j}^{\prime}, 0\right)$. C'est donc la fonction de Hilbert-Samuel de l'anneau local $\mathbb{K}\{t, X\} / I_{j, A^{j}}$. Dans ces notations, la suite $\mathcal{H}_{X, \varphi^{2}}^{i}$ est la suite $\left(H_{0}, H_{A_{1}}, \ldots, H_{A_{1}, \ldots, A_{2}}\right)$, où $H_{0}$ est la fonction de Hilbert-Samuel de $\left(Z_{0}^{\prime}, 0\right)=(\mathbb{K}, 0) \times(X, 0)$. Soit $j, 1 \leqslant j \leqslant i$. Désignons par $V_{j}$ l'adhérence de Zariski de $P_{j}(V) \subset \mathbb{K}^{n j}$, où $P_{j}: \mathbb{K}^{n i} \rightarrow \mathbb{K}^{n j}$ désigne la projection oublie des $n(i-j)$ derniers termes. $V_{j}$ est donc une sous-variété irréductible de $\mathbb{K}^{n j}$. Considérons maintenant une base standard, $f_{1}(X), \ldots, f_{p_{0}}(X) \in \mathbb{K}[[X]]$ de $I_{0}$, où $I_{0}$ est l'idéal définissant $\mathcal{O}_{X, 0}$ comme $\mathbb{K}\{X\} / I_{0}$. Puis posons :

$$
F_{k, 0}(t, X)=f_{k}(X) \in \mathbb{K}[[X]], \quad 1 \leqslant k \leqslant p_{0} .
$$

Soit alors :

$$
F_{k, 1}(t, X)=\frac{F_{k, 0}\left(t, t\left(Y_{1}+X\right)\right)}{t^{\left|\nu\left(F_{k, 0}\right)\right|}} \in \mathbb{K}\left[Y_{1}\right][[t, X]], 1 \leqslant k \leqslant p_{0} .
$$

Prenant la classe des coefficients des $F_{k, 1}$ dans $\mathbb{K}\left[V_{1}\right]$, on obtient des éléments $\bar{F}_{k, 1}(t, X) \in \mathbb{K}\left[V_{1}\right][[t, X]]$. On notera $R_{1}$ l'idéal de $\mathbb{K}\left[V_{1}\right][[t, X]]$ engendré par les $\bar{F}_{k, 1}(t, X)$ et soit $N\left(R_{1}\right)$ son diagramme des exposants initiaux. Désignons par $\beta_{1,1}, \ldots, \beta_{p_{1}, 1}$ les sommets de $N\left(R_{1}\right)$. D'après le lemme précédent il existe une sous-variété propre $V_{1}^{\prime}$ de $V_{1}$ et des éléments $G_{1,1}, \ldots, G_{p_{1}, 1}$ $\in R_{1} \subset \mathbb{K}\left[V_{1}\right][[t, X]]$ tels que :

$$
\begin{aligned}
& \text { - } \forall A^{1} \in V_{1}-V_{1}^{\prime}, N\left(R_{1}\right)=N\left(R_{1, A^{1}}\right) ; \\
& \text { - } \forall A^{1} \in V_{1}-V_{1}^{\prime}, \forall k, 1 \leqslant k \leqslant p_{1}, \beta_{k, 1}=\nu\left(G_{k, 1}\right)=\nu\left(G_{k, 1}\left(A^{1}, t, X\right)\right) .
\end{aligned}
$$


Posons ensuite :

$$
F_{k, 2}(t, X)=\frac{G_{k, 1}\left(t, t\left(Y_{2}+X\right)\right)}{t^{\left|\nu\left(G_{k, 1}\right)\right|}} \in \mathbb{K}\left[V_{1}\right]\left[Y_{2}\right][[t, X]], 1 \leqslant k \leqslant p_{1} .
$$

Prenant la classe des coefficients des $F_{k, 2}$ dans $\mathbb{K}\left[V_{2}\right]$, on obtient des éléments $\bar{F}_{k, 2}(t, X) \in \mathbb{K}\left[V_{2}\right][[t, X]]$. On notera $R_{2}$ l'idéal de $\mathbb{K}\left[V_{2}\right][[t, X]]$ engendré par les $\bar{F}_{k, 2}(t, X)$ et soit $N\left(R_{2}\right)$ son diagramme des exposants initiaux. Désignons par $\beta_{1,2}, \ldots, \beta_{p_{2}, 2}$ les sommets de $N\left(R_{2}\right)$. À nouveau le lemme 4.10 assure l'existence d'une sous-variété propre $V_{2}^{\prime}$ de $V_{2}$ et d'éléments $G_{1,2}, \ldots, G_{p_{2}, 2} \in R_{2} \subset \mathbb{K}\left[V_{2}\right][[t, X]]$ tels que :

$$
\begin{aligned}
- & \forall A^{2}=\left(A_{1}, A_{2}\right) \in V_{2}-V_{2}^{\prime}, N\left(R_{2}\right)=N\left(R_{2, A^{2}}\right) ; \\
- & \forall A^{2}=\left(A_{1}, A_{2}\right) \in V_{2}-V_{2}^{\prime}, \forall k, 1 \leqslant k \leqslant p_{2}, \beta_{k, 2}=\nu\left(G_{k, 2}\right)= \\
& \nu\left(G_{k, 2}\left(A^{2}, t, X\right)\right) .
\end{aligned}
$$

On continue le procédé par récurrence. Soit $j<i$. Ayant déterminé une sous-variété propre $V_{j}^{\prime}$ de $V_{j}$ et des éléments $\bar{F}_{k, j}(t, X) \in \mathbb{K}\left[V_{j}\right][[t, X]]$, $1 \leqslant k \leqslant p_{j-1}$, engendrant un idéal $R_{j}$, il existe des éléments $G_{1, j}, \ldots, G_{p_{j}, j}$ $\in R_{j} \subset \mathbb{K}\left[V_{j}\right][[t, X]]$ tels que :

$$
\begin{aligned}
- & \forall A^{j}=\left(A_{1}, \ldots, A_{j}\right) \in V_{j}-V_{j}^{\prime}, N\left(R_{j}\right)=N\left(R_{j, A^{\jmath}}\right) ; \\
- & \forall A^{j}=\left(A_{1}, \ldots, A_{j}\right) \in V_{j}-V_{j}^{\prime}, \forall k, 1 \leqslant k \leqslant p_{j}, \beta_{k, j}=\nu\left(G_{k, j}\right)= \\
& \nu\left(G_{k, j}\left(A^{j}, t, X\right)\right),
\end{aligned}
$$

où les $\beta_{k, j}$ sont les sommets de $N\left(R_{j}\right)$.

On pose alors :

$$
F_{k, j+1}(t, X)=\frac{G_{k, j}\left(t, t\left(Y_{j+1}+X\right)\right)}{t^{\left|\nu\left(G_{k, j}\right)\right|}} \in \mathbb{K}\left[V_{j}\right]\left[Y_{j+1}\right][[t, X]], 1 \leqslant k \leqslant p_{j} .
$$

On note $\bar{F}_{k, j+1}(t, X) 1 \leqslant k \leqslant p_{j}$, les éléments de $\mathbb{K}\left[V_{j+1}\right][[t, X]]$ obtenus en prenant les classes des coefficients. Soient $R_{j+1}$ l'idéal de $\mathbb{K}\left[V_{j+1}\right][[t, X]]$ engendré par les $\bar{F}_{k, j+1}(t, X)$ et $N\left(R_{j+1}\right)$ son diagramme des exposants initiaux. Le Lemme 4.10 assure l'existence d'éléments $G_{k, j+1} \in R_{j+1}$, $1 \leqslant k \leqslant p_{j+1}$ et d'une sous-variété prorpre $V_{j}^{\prime}$ de $V_{j}$ tels que :

$$
\begin{aligned}
& -\forall A^{j+1}=\left(A_{1}, \ldots, A_{j+1}\right) \in V_{j+1}-V_{j+1}^{\prime}, N\left(R_{j+1}\right)=N\left(R_{j+1, A^{j+1}}\right) ; \\
& -\forall A^{j+1}=\left(A_{1}, \ldots, A_{j+1}\right) \in V_{j+1}-V_{j+1}^{\prime}, \forall k, 1 \leqslant k \leqslant p_{j+1}, \beta_{k, j+1} \\
& \quad=\nu\left(G_{k, j+1}\right)=\nu\left(G_{k, j+1}\left(A^{j+1}, t, X\right)\right),
\end{aligned}
$$


où les $\beta_{k, j+1}$ sont les sommets de $N\left(R_{j+1}\right)$.

On pose alors :

$$
\left.V^{\prime}=\bigcup_{1 \leqslant j \leqslant i}\left(\left(V_{j}^{\prime} \times \mathbb{K}^{n(i-j)}\right) \cap V\right)\right) .
$$

Alors $V^{\prime}$ est une sous-variété propre de $V$. Soient alors $A^{i}=\left(A_{1}, \ldots, A_{i}\right)$ (resp. $\left.B^{i}=\left(B_{1}, \ldots, B_{i}\right)\right) \in V-V^{\prime}$. Puisque le diagramme des exposants initiaux détermine la fonction de Hilbert-Samuel, il nous suffit pour conclure de voir que :

$$
N\left(I_{j, A^{\jmath}}\right)=N\left(I_{j, B^{\jmath}}\right), \quad 1 \leqslant j \leqslant i .
$$

Pour cela du fait que les $F_{k, 0}$ sont une base standard de $I_{0}$, leurs transformées strictes engendrent $I_{1, A^{1}}$ pour tout $A^{1} \in V_{1}$. Par conséquent pour tout $A^{1} \in V_{1}, I_{1, A^{1}}$ est engendré par les $\bar{F}_{k, 1}\left(A^{1}, t, X\right), 1 \leqslant k \leqslant p_{0}$. Maintenant si $A^{1} \in V_{1}-V_{1}^{\prime}$, par construction même les $G_{k, 1}\left(A^{1}, t, X\right)$ sont une base standard de $I_{1, A^{1}}=R_{1, A^{1}}$. Par suite $N\left(I_{1, A^{1}}\right)=N\left(I_{1, B^{1}}\right)=N\left(R_{1}\right)$ et donc $H_{A^{1}}=H_{B^{1}}$. De plus, les transformées strictes des $G_{k, 1}\left(A^{1}, t, X\right)$ engendrent $I_{2, A_{1}, A_{2}}, \forall A_{1} \in V_{1}-V_{1}^{\prime}, A_{2} \in \mathbb{K}^{n}$. Par conséquent les $\bar{F}_{k, 2}\left(A_{1}, A_{2}, t, X\right), 1 \leqslant k \leqslant p_{1}$, engendrent $I_{2, A_{1}, A_{2}}, \forall A^{1} \in V_{1}-V_{1}^{\prime}, A^{2} \in \mathbb{K}^{n}$. Par suite, $\forall A_{1} \in V_{1}-V_{1}^{\prime}, A_{2} \in \mathbb{K}^{n}, R_{2, A_{1}, A_{2}}=I_{2, A_{1}, A_{2}}$. Par conséquent :

$$
N\left(I_{2, A_{1}, A_{2}}\right)=N\left(I_{2, B_{1}, B_{2}}\right)=N\left(R_{2}\right) \text {, et } H_{A_{1}, A_{2}}=H_{B_{1}, B_{2}} .
$$

Par construction, $\forall\left(A_{1}, A_{2}\right) \in V_{2}-V_{2}^{\prime}$, les $G_{k, 2}\left(A_{1}, A_{2}, t, X\right)$ sont une base standard de $I_{2, A_{1}, A_{2}}$. Leurs transformées strictes engendrent donc $I_{3, A^{3}}$, $\forall A^{3} \in\left(V_{2}-V_{2}^{\prime}\right) \times \mathbb{K}^{n}$. Par suite, les $F_{k, 3}\left(A^{3}, t, X\right)$ engendrent $I_{3, A^{3}}, \forall A^{3} \in$ $\left(V_{2}-V_{2}^{\prime}\right) \times \mathbb{K}^{n}$. Donc $\forall A^{3} \in V_{3}-V_{3}^{\prime}, N\left(I_{3, A^{3}}\right)=N\left(R_{3}\right)=N\left(R_{3, A^{3}}\right)$. D'où :

$$
H_{A^{3}}=H_{B^{3}} .
$$

On continue ainsi par récurrence jusqu'à l'ordre $i$. Notons que pour tout $\varphi^{i} \in V-V^{\prime}$,

$$
\mathcal{N}_{X, \varphi^{\imath}}^{i}=\left(N\left(I_{1, A_{1}}\right), \ldots, N\left(I_{i, A^{2}}\right)\right)=\left(N\left(R_{1}\right), \ldots, N\left(R_{i}\right)\right)
$$

LEMME 4.12. - Soient $i \in \mathbb{N}^{*}$ et $V$ une sous-variété algébrique irréductible de $\mathcal{A}_{\left(\mathbb{K}^{n}, 0\right)}^{i}=\mathbb{K}^{n i}$. Soit $V^{\prime}$ comme dans le Lemme 4.11. On a :

$$
\forall \varphi^{i} \in V-V^{\prime}, \quad \forall \theta^{i} \in V^{\prime}, \mathcal{N}_{X, \varphi^{\imath}}^{i} \leqslant \mathcal{N}_{X, \theta^{\imath}}^{i} .
$$

Preuve. - On procède par récurrence sur $i$. Pour $i=1$, la propriété résulte du Lemme 4.10 puisque pour tout $A_{1} \in V, I_{1, A_{1}}=R_{1, A_{1}}$. Nous 
supposerons donc $i>1$ et la propriété établie pour $i-1$. Pour établir la propriété au rang i, on raisonne par récurrence sur $m$ la dimension de $V$. Si $m=0$, le résultat est évident. On peut donc supposer $m>0$ et la propriété établie pour toute sous-variété irréductible de $\mathbb{K}^{n i}$ de dimension $\leqslant m-1$. Soient $V, V^{\prime}$ comme dans 4.11. Considérons la décomposition en composantes irréductibles de $V^{\prime}$ :

$$
V^{\prime}=\bigcup_{1 \leqslant l \leqslant t} V^{\prime l} .
$$

Le Lemme 4.11 fournit pour chaque $l$ une sous-variété propre $V^{\prime \prime l}$ telle que :

$$
\forall \varphi^{i}, \forall \theta^{i} \in V^{\prime l}-V^{\prime \prime l}, \mathcal{N}_{X, \varphi^{\prime \imath}}^{i}=\mathcal{N}_{X, \theta^{\prime \imath}}^{i}
$$

Notre hypothèse de récurrence sur la dimension nous dit que :

$$
\forall \varphi^{i} \in V^{\prime l}-V^{\prime \prime l}, \forall \theta^{i} \in V^{\prime \prime l}, \mathcal{N}_{X, \varphi^{\prime \imath}}^{i} \leqslant \mathcal{N}_{X, \theta^{\prime \imath}}^{i} .
$$

Par conséquent pour établir la propriété au rang $i$, on peut supposer que $V^{\prime}$ est irréductible et qu'il existe une sous-variété propre $V^{\prime \prime}$ de $V^{\prime}$ comme dans 4.11. Il s'agit alors de voir que :

$$
\forall \varphi^{i} \in V-V^{\prime}, \forall \theta^{i} \in V^{\prime}-V^{\prime \prime}, \mathcal{N}_{X, \varphi^{\imath}}^{i} \leqslant \mathcal{N}_{X, \theta^{2}}^{i}
$$

Maintenant par hypothèse de récurrence sur $i$, notant $\varphi^{i-1}$ et $\theta^{i-1}$ les tronqués à l'ordre $i-1$ de $\varphi^{i}$ et $\theta^{i}$, on a :

$$
\mathcal{N}_{X, \varphi^{2-1}}^{i-1} \leqslant \mathcal{N}_{X, \theta^{2-1}}^{i-1}
$$

Si l'inégalité ci-dessus est stricte, par definition de l'ordre lexicographique on a alors aussi une inégalité stricte au rang $i$. On peut donc supposer que :

$$
\mathcal{N}_{X, \varphi^{2-1}}^{i-1}=\mathcal{N}_{X, \theta^{2-1}}^{i-1}
$$

La preuve du Lemme 4.11, appliquée à $V^{\prime}$, nous dit qu'il existe des sousvariétés propres $V_{j}^{\prime \prime}$ de $V_{j}^{\prime}, 1 \leqslant j \leqslant i$, des éléments $\bar{F}_{k, j}^{\prime} \in \mathbb{K}\left[V_{j}^{\prime}\right][[t, X]]$, $1 \leqslant k \leqslant p_{j-1}^{\prime}$, engendrant un idéal $R_{j}^{\prime}$ dont le diagramme des exposants initiaux est noté $N\left(R_{j}^{\prime}\right)$, et des éléments $G_{k, j}^{\prime} \in R_{j}^{\prime}$ tels que :

$$
\begin{aligned}
- & \forall B^{j}=\left(B_{1}, \ldots, B_{j}\right) \in V_{j}^{\prime}-V_{j}^{\prime \prime}, N\left(R_{j}^{\prime}\right)=N\left(R_{j, B^{\jmath}}^{\prime}\right) \\
- & \forall B^{j}=\left(B_{1}, \ldots, B_{j}\right) \in V_{j}^{\prime}-V_{j}^{\prime \prime}, \nu\left(G_{k, j}^{\prime}\right)=\nu\left(G_{k, j}^{\prime}\left(B^{j}, t, X\right)\right), 1 \leqslant k \leqslant \\
& p_{j}^{\prime}, \text { où les } \beta_{k, j}^{\prime} \text { sont les sommets de } N\left(R_{j}^{\prime}\right) .
\end{aligned}
$$


On a de plus :

$$
F_{k, j+1}^{\prime}(t, X)=\frac{G_{k, j}^{\prime}\left(t, t\left(Y_{j+1}+X\right)\right)}{t^{\left|\nu\left(G_{k, j}^{\prime}\right)\right|}} \in \mathbb{K}\left[V_{j}^{\prime}\right]\left[Y_{j+1}\right][[t, X]] .
$$

Dont les classes donnent les $\bar{F}_{k, j+1}^{\prime} \in \mathbb{K}\left[V_{j+1}^{\prime}\right][[t, X]]$. Par hypothèse, on a $p_{j-1}^{\prime}=p_{j-1}$ et $\beta_{k, j}^{\prime}=\beta_{k, j}, j \leqslant i-1$. Pour conclure il suffit d'établir la propriété suivante :

Sous-LEMME 4.13. -

Il existe des éléments $\tilde{F}_{k, i}^{\prime} \in \mathbb{K}[V][[t, X]], 1 \leqslant k \leqslant p_{i-1}$, tels que :

1) L'idéal de $\mathbb{K}[[t, X]]$ engendré par les $\tilde{F}_{k, i}^{\prime}\left(B_{1}, \ldots, B_{i}, t, X\right)$ est le même que celui engendré par les $\bar{F}_{k, i}^{\prime}\left(B_{1}, \ldots, B_{i}, t, X\right), \forall B^{i}=\left(B_{1}, \ldots, B_{i}\right)$ $\in V^{\prime}-W^{\prime \prime}$, où $W^{\prime \prime}$ est une sous-variété propre de $V^{\prime}$;

2) $\tilde{F}_{k, i}^{\prime}\left(A_{1}, \ldots, A_{i-1}, A_{i}, t, X\right) \in I_{i, A_{1}, \ldots, A_{\imath-1}, A_{2}}, \forall A^{i}=\left(A_{1}, \ldots, A_{i}\right) \in$ $V$.

En effet supposons (4.13) établie. Notons $\tilde{R}_{i}$ l'idéal de $\mathbb{K}[V][[t, X]]$ engendré par les $\tilde{F}_{k, i}^{\prime}$ et par $N\left(\tilde{R}_{i}\right)$ son diagramme des exposants initiaux. Par le second point de (4.13), on a pour tout $A^{i} \in V, \tilde{R}_{i, A^{2}} \subset I_{i, A^{2}}$. Donc $N\left(I_{i, A^{2}}\right) \leqslant N\left(\tilde{R}_{i, A^{2}}\right)$. Maintenant par le Lemme 4.10 , il existe une sous variété propre $W^{\prime}$ de $V$ telle que $\forall A^{i} \in V-W^{\prime}, N\left(\tilde{R}_{i, A^{2}}\right)=N\left(\tilde{R}_{i}\right)$. Par irréductibilité de $V, V^{\prime} \cup W^{\prime} \varsubsetneqq V$. Considérant un point $A^{i} \in V-\left(V^{\prime} \cup W^{\prime}\right)$ on a donc :

$$
N\left(R_{i}\right)=N\left(I_{i, A^{2}}\right) \leqslant N\left(\tilde{R}_{i, A^{2}}\right)=N\left(\tilde{R}_{i}\right)
$$

Maintenant par le premier point de (4.13), considérons un point $B^{i}$ de $V^{\prime}-$ $\left(V^{\prime \prime} \cup W^{\prime \prime}\right)$ (qui est nécessairement non vide par irréductibilité de $V^{\prime}$ ), on a :

$$
N\left(R_{i}^{\prime}\right)=N\left(I_{i, B^{\imath}}\right)=N\left(\tilde{R}_{i, B^{2}}\right) .
$$

Par suite par le Lemme $4.10, N\left(\tilde{R}_{i}\right) \leqslant N\left(R_{i}^{\prime}\right)$. D'oú $N\left(R_{i}\right) \leqslant N\left(R_{i}^{\prime}\right)$, ce que nous cherchions à démontrer. Il nous reste donc à prouver (4.13).

Nous allons à nouveau procéder par récurrence sur $i$. Les notations étant celles de la preuve du Lemme 4.11 on a :

$$
F_{k, 1}(t, X)=\frac{F_{k, 0}\left(t, t\left(Y_{1}+X\right)\right)}{t^{\left|\nu\left(F_{k, 0}\right)\right|}} \in \mathbb{K}\left[Y_{1}\right][[t, X]], 1 \leqslant k \leqslant p_{0} .
$$


Les $\bar{F}_{k, 1}^{\prime}$ sont les classes dans $\mathbb{K}\left[V_{1}^{\prime}\right][[t, X]]$ des $F_{k, 1}$. Posons : $\tilde{F}_{k, 1}^{\prime}(t, X)=$ $\bar{F}_{k, 1}(t, X) \in \mathbb{K}\left[V_{1}\right][[t, X]]$. (4.13) est alors claire pour $i=1$. D'autre part, il existe des $\bar{U}_{k, l}^{1} \in \mathbb{K}\left[V_{1}^{\prime}\right][[t, X]]$ tels que :

$$
G_{k, 1}^{\prime}=\sum_{l=1}^{p_{0}} \bar{U}_{k, l}^{1} \bar{F}_{l, 1}^{\prime} \in \mathbb{K}\left[V_{1}^{\prime}\right][[t, X]] .
$$

Posons :

$$
G_{k, 1}^{\prime \prime}=\sum_{l=1}^{p_{0}} U_{k, l}^{1} \tilde{F}_{l, 1}^{\prime} \in \mathbb{K}\left[V_{1}\right][[t, X]]
$$

où les $U_{k, l}^{1}$ représentent les $\bar{U}_{k, l}^{1}$. Nous allons construire des $\tilde{G}_{k, 1}^{\prime} \in \mathbb{K}\left[V_{1}\right][[t, X]]$, $1 \leqslant k \leqslant p_{1}$, tels que :

$$
\begin{aligned}
& -\tilde{G}_{k, 1}^{\prime} \in R_{1} ; \\
& -\nu\left(\tilde{G}_{k, 1}^{\prime}\right)=\beta_{k, 1} ; \\
& -\forall B_{1} \in V_{1}^{\prime}-V_{1}^{\prime \prime}, \nu\left(\tilde{G}_{k, 1}^{\prime}\left(B_{1}, t, X\right)\right)=\nu\left(G_{k, 1}^{\prime}\left(B_{1}, t, X\right)\right)=\beta_{k, 1} ; \\
& -\forall B_{1} \in V_{1}^{\prime}-V_{1}^{\prime \prime}, \tilde{G}_{k, 1}^{\prime}\left(B_{1}, t, X\right)=G_{k, 1}^{\prime}\left(B_{1}, t, X\right) \text { modulo un élément } \\
& \quad \text { de } \mathbb{K}^{*} .
\end{aligned}
$$

On procède par récurrence sur $k$. On a d'abord :

$$
\nu\left(G_{1,1}^{\prime \prime}\right) \leqslant \nu\left(G_{1,1}^{\prime}\right)=\beta_{1,1} .
$$

Mais $\nu\left(G_{1,1}^{\prime \prime}\right) \in N\left(R_{1}\right)$. Donc $\nu\left(G_{1,1}^{\prime \prime}\right) \geqslant \beta_{1,1}$. Par suite $\nu\left(G_{1,1}^{\prime \prime}\right)=\nu\left(G_{1,1}^{\prime}\right)$. Posons $\tilde{G}_{1,1}^{\prime}=G_{1,1}^{\prime \prime} \in \mathbb{K}\left[V_{1}\right][[t, X]]$. Soit $k, 1 \leqslant k<p_{1}$, supposons avoir construit $\tilde{G}_{1,1}^{\prime}, \ldots, \tilde{G}_{k, 1}^{\prime} \in \mathbb{K}\left[V_{1}\right][[t, X]]$ satisfaisant les propriétés ci-dessus. On a : $\nu\left(G_{k+1,1}^{\prime \prime}\right) \leqslant \nu\left(G_{k+1,1}^{\prime}\right)=\beta_{k+1,1}$. Mais $\nu\left(G_{k+1,1}^{\prime \prime}\right) \in N\left(R_{1}\right)$. Donc :

$$
\nu\left(G_{k+1,1}^{\prime \prime}\right) \in \cup_{k+1 \leqslant s \leqslant p_{1}}\left(\beta_{s, 1}+\mathbb{N}^{n+1}\right),
$$

ou bien

$$
\nu\left(G_{k+1,1}^{\prime \prime}\right) \in \cup_{1 \leqslant s \leqslant k}\left(\beta_{s, 1}+\mathbb{N}^{n+1}\right) .
$$

Dans le premier cas on a $\nu\left(G_{k+1,1}^{\prime \prime}\right) \geqslant \beta_{k+1,1}$ et donc $\nu\left(G_{k+1,1}^{\prime \prime}\right)=\beta_{k+1,1}$. On pose alors $\tilde{G}_{k+1,1}^{\prime}=G_{k+1,1}^{\prime \prime}$. Dans le second cas si $\nu\left(G_{k+1,1}^{\prime \prime}\right)<\nu\left(G_{k+1,1}^{\prime}\right)$, on écrit :

$$
\nu\left(G_{k+1,1}^{\prime \prime}\right)=\beta_{l, 1}+\gamma, \text { avec } 1 \leqslant l \leqslant k \text { et } \gamma \in \mathbb{N}^{n+1} .
$$


On considère alors :

$G_{k+1,1}^{\prime \prime \prime}(t, X)=\tilde{g}_{\beta_{l, 1}, l, 1}^{\prime}\left(Y_{1}\right) G_{k+1,1}^{\prime \prime}(t, X)-g_{\beta_{l, 1}+\gamma, k+1,1}^{\prime \prime}\left(Y_{1}\right) t^{\gamma_{1}} X^{\gamma^{\prime}} \tilde{G}_{l, 1}^{\prime}(t, X)$.

Où $\gamma=\left(\gamma_{1}, \gamma^{\prime}\right)$ et $\tilde{g}_{\beta_{l, 1}, l, 1}^{\prime}\left(Y_{1}\right)$ est le coefficient dans le développement de $\tilde{G}_{l, 1}^{\prime}$ correspondant au monôme $t^{\beta_{l, 1,1}} X^{\beta_{l, 1}^{\prime}}$ avec $\beta_{l, 1}=\left(\beta_{l, 1,1}, \beta_{l, 1}^{\prime}\right)$ et $g_{\beta_{l, 1}+\gamma, k+1,1}^{\prime \prime}\left(Y_{1}\right)$ est celui correspondant au monôme $t^{\beta_{l, 1,1}+\gamma_{1}} X^{\beta_{l, 1}^{\prime}+\gamma^{\prime}}$ dans le developpement de $G_{k+1,1}^{\prime \prime}$. On a alors :

$$
(* *) \quad \nu\left(G_{k+1,1}^{\prime \prime}\right)<\nu\left(G_{k+1,1}^{\prime \prime \prime}\right) \leqslant \nu\left(G_{k+1,1}^{\prime}\right)=\beta_{k+1,1} .
$$

De plus :

- $\forall B_{1} \in V_{1}^{\prime}-V_{1}^{\prime \prime}, G_{k+1,1}^{\prime \prime \prime}\left(B_{1}, t, X\right)=G_{k+1,1}^{\prime}\left(B_{1}, t, X\right)$ à la multiplication près par un élément de $\mathbb{K}^{*}$;

$$
\begin{aligned}
& -\forall B_{1} \in V_{1}^{\prime}-V_{1}^{\prime \prime}, \nu\left(G_{k+1,1}^{\prime \prime \prime}\left(B_{1}, t, X\right)\right)=\beta_{k+1,1} ; \\
& -G_{k+1,1}^{\prime \prime \prime} \in R_{1} .
\end{aligned}
$$

On recommence alors le même raisonnement avec $G_{k+1,1}^{\prime \prime \prime}$ au lieu de $G_{k, 1}^{\prime}$. Puisque la première inégalité de $(* *)$ est stricte, au bout d'un nombre fini d'étapes, on aura construit un élément $\tilde{G}_{k+1,1}^{\prime} \in \mathbb{K}\left[V_{1}\right][[t, X]]$ possédant les propriétés désirées.

Maintenant ayant construit $\tilde{G}_{1,1}^{\prime}, \ldots, \tilde{G}_{p_{1}, 1}^{\prime}$, on pose :

$$
\tilde{F}_{k, 2}^{\prime}(t, X)=\frac{\tilde{G}_{k, 1}^{\prime}\left(t, t\left(Y_{2}+X\right)\right)}{t^{\left|\beta_{k, 1}\right|}} \in \mathbb{K}\left[V_{1}\right]\left[Y_{2}\right][[t, X]], 1 \leqslant k \leqslant p_{1}
$$

et on note encore $\tilde{F}_{k, 2}^{\prime}$ les éléments de $\mathbb{K}\left[V_{2}\right][[t, X]]$ obtenus en prenant la classe des coefficients dans $\mathbb{K}\left[V_{2}\right]$. Par construction même $\tilde{F}_{k, 2}^{\prime}\left(B_{1}, B_{2}, t, X\right)$ et $\bar{F}_{k, 2}^{\prime}\left(B_{1}, B_{2}, t, X\right)$ diffèrent au plus multiplicativement d'une constante non nulle pour tout $\left(B_{1}, B_{2}\right) \in V_{2}^{\prime}-V_{2}^{\prime \prime}$. Le premier point de (4.13) en découle pour $i=2$. Concernant le second point, pour tout $A_{1} \in V_{1}$, $\tilde{G}_{k, 1}^{\prime}\left(A_{1}, t, X\right) \in R_{1, A_{1}}=I_{1, A_{1}}$. Donc pour tout $A_{2} \in \mathbb{K}^{n}$, leurs transformées strictes :

$$
\frac{\tilde{G}_{k, 1}^{\prime}\left(A_{1}, t, t\left(A_{2}+X\right)\right)}{t^{\left|\nu\left(\tilde{G}_{k, 1}^{\prime}\left(A_{1}, t, X\right)\right)\right|}}
$$

sont des éléments de $I_{2, A_{1}, A_{2}}$. Mais :

$$
\left|\nu\left(\tilde{G}_{k, 1}^{\prime}\left(A_{1}, t, X\right)\right)\right| \geqslant\left|\nu\left(\tilde{G}_{k, 1}^{\prime}(t, X)\right)\right|=\left|\beta_{k, 1}\right| .
$$


Donc les $\tilde{F}_{k, 2}^{\prime}\left(A_{1}, A_{2}, t, X\right)$ sont des multiples de ces transformées strictes et sont par suite des éléments de $I_{2, A_{1}, A_{2}}$. Donc (4.13) est établie pour $i=2$.

Maintenant pour passer du rang $i-1$ au rang $i$, on raisonne exactement comme nous venons de le faire pour le passage du rang 1 au rang 2 . On considère l'idéal $\tilde{R}_{i-1}$ de $\mathbb{K}\left[V_{i-1}\right][[t, X]]$ engendré par les $\tilde{F}_{k, i-1}^{\prime}$, ainsi que l'idéal $\tilde{R}_{i-1}^{\prime}$ de $\mathbb{K}\left[V_{i-1}^{\prime}\right][[t, X]]$ engendré par les classes des $\tilde{F}_{k, i-1}^{\prime}$. On applique le Lemme 4.10 à $\tilde{R}_{i-1}^{\prime}$, ce qui fournit des éléments $\hat{G}_{k, i-1}^{\prime}$ analogues des $G_{k, 1}^{\prime}$. Ceux-ci permettent de construire par le même procédé que dans le passage du rang 1 au rang $2 \operatorname{des} \tilde{G}_{k, i-1}^{\prime} \in \mathbb{K}\left[V_{i-1}\right][[t, X]], 1 \leqslant k \leqslant p_{i-1}$, tels que :

$-\nu\left(\tilde{G}_{k, i-1}^{\prime}\right)=\beta_{k, i-1} ;$

- $\nu\left(\tilde{G}_{k, i-1}^{\prime}\left(B_{1}, \ldots, B_{i-1}, t, X\right)\right)=\beta_{k, i-1}$ pour tout $\left(B_{1}, \ldots, B_{i-1}\right)$ dans un ouvert de Zariski non vide de $V_{i-1}^{\prime}$;

- $\tilde{G}_{k, i-1}^{\prime}\left(B_{1}, \ldots, B_{i-1}, t, X\right)=\hat{G}_{k, i-1}^{\prime}\left(B_{1}, \ldots, B_{i-1}, t, X\right)$ à la multiplication près par un élément de $\mathbb{K}^{*}$, pour tout $\left(B_{1}, \ldots, B_{i-1}\right)$ dans un ouvert de Zariski non vide de $V_{i-1}^{\prime}$;

- $\tilde{G}_{k, i-1}^{\prime} \in \tilde{R}_{i-1}$.

Ceux-ci permettent de définir les $\tilde{F}_{k, i}^{\prime}$ par la formule :

$$
\tilde{F}_{k, i}^{\prime}(t, X)=\frac{\tilde{G}_{k, i-1}^{\prime}\left(t, t\left(Y_{i}+X\right)\right)}{t^{\left|\beta_{k, 2-1}\right|}} \in \mathbb{K}\left[V_{i}\right][[t, X]], 1 \leqslant k \leqslant p_{i-1} .
$$

On vérifie alors les propriétés de (4.13) comme plus haut.

Pour finir la preuve du théorème de semi-continuité, il nous suffit de dire que celui-ci résulte des Lemmes 4.11 et 4.12 .

Preuve de 1.8. - La semi-continuité pour la topologie de Zariski d'une fonction $\mathcal{F}^{i}$ de $\mathcal{A}_{\left(\mathbb{K}^{n}, 0\right)}^{i}$ dans un ensemble ordonné $\Lambda$ entraine que pour tout $\lambda \in \Lambda$ l'ensemble :

$$
S_{\lambda}^{i}=\left\{\varphi^{i} \in \mathcal{A}_{\left(\mathbb{K}^{n}, 0\right)}^{i} \mid \mathcal{F}^{i}\left(\varphi^{i}\right) \geqslant \lambda\right\}
$$

est une sous-variété algébrique de $\mathcal{A}_{\left(\mathbb{K}^{n}, 0\right)}^{i}$. D'autre part d'après le théorème de semi-continuité $\varphi^{i} \rightarrow \mathcal{N}_{X, \varphi^{2}}^{i}$ ne prend qu'un nombre fini de valeurs $\mathcal{N}_{i, j}$, $1 \leqslant j \leqslant l_{i}$, sur $\overline{\Pi^{i}\left(\mathcal{A}_{(X, x)}\right)}$. On pose alors :

$$
U_{i, j}=\left\{\varphi^{i} \in \overline{\Pi^{i}\left(\mathcal{A}_{(X, x)}\right)} \mid \mathcal{N}_{X, \varphi^{2}} \geqslant \mathcal{N}_{i, j}\right\} \text { et } W_{i, j}=\cup_{j^{\prime} \neq j} U_{i, j} .
$$


Les propriétés de 1.8 sont alors claires puisque le diagramme des exposants initiaux détermine la fonction de Hilbert-Samuel ainsi que la multiplicité.

Remarque 4.14 . - 1) Il est naturel de se demander aussi si les fonctions $\varphi^{i} \rightarrow \mathcal{M}_{X, \varphi^{i}}^{i}$ et $\varphi^{i} \rightarrow \mathcal{H}_{X, \varphi^{2}}^{i}$ sont semi-continues supérieurement pour la topologie de Zariski sur $\mathcal{A}_{\left(\mathbb{K}^{n}, 0\right)}^{i}$. Ceci est au moins le cas pour les hypersurfaces. Cependant l'auteur n'a pas réussi pour le moment à le prouver dans le cas général.

2) On peut raffiner la partition $S_{i, j}$ ci-dessus en écrivant $S_{i, j}=$ $\cup_{1 \leqslant k \leqslant t_{\imath, j}} U_{i, j, k}-W_{i, j, k}$, où $U_{i, j, k}$ et $W_{i, j, k}$ sont des variétés algébriques. De telle sorte que pour chaque $k$, il existe $\operatorname{des} F_{k, l}(t, X) \in R\left(U_{i, j, k}, W_{i, j, k}\right)[[t, X]]$, $R\left(U_{i, j, k}, W_{i, j, k}\right)$ désignant les fonctions régulières sur $U_{i, j, k}$ à pôles dans $W_{i, j, k}$, telles que $\forall A^{i} \in U_{i, j, k}-W_{i, j, k}$ les $F_{k, l}\left(A^{i}, t, X\right)$ soient la base standard distinguée de $I_{i, A^{2}}$.

\section{Strates Principales et volume motivique}

Soient $(X, x)$ un germe de $\mathbb{K}$-espace analytique et $i \geqslant 1$ un entier. D'après les résultats de la section précédente, on dispose d'une partition :

$$
\mathbb{K}^{n i}=\bigcup_{1 \leqslant l \leqslant k_{\imath}} S_{i, l}
$$

où les $S_{i, l}$ sont des sous-ensembles constructibles de $\mathbb{K}^{n i}$ tels que :

- sur chaque $S_{i, l}, \mathcal{N}_{X, \varphi^{2}}^{i}=\left(N_{0, \varphi^{2}}, \ldots, N_{i, \varphi^{2}}\right)$ est constante égale à $\mathcal{N}_{X, l}^{i}=$ $\left(N_{0}^{l}, \ldots, N_{i}^{l}\right)$

- Si $l \neq l^{\prime}, \mathcal{N}_{X, l}^{i} \neq \mathcal{N}_{X, l^{\prime}}^{i}$

Par voie de conséquences $\mathcal{M}_{X, \varphi^{2}}^{i}$ et $\mathcal{H}_{X, \varphi^{i}}^{i}$ sont constantes sur chaque $S_{i, l}$. On notera respectivement $\mathcal{M}_{X, l}^{i}=\left(m_{0}^{l}, \ldots, m_{i}^{l}\right)$ et $\mathcal{H}_{X, l}^{i}=\left(H_{0}^{l}, \ldots, H_{i}^{l}\right)$ la valeur de ces constantes.

DÉfINITION 5.1. - Soient $(X, x)$ un germe de $\mathbb{K}$-espace analytique et $i \geqslant 1$ un entier donné. On dit qu'une strate $S_{i, l}$ de la statification de $\mathbb{K}^{n i}$ par les suites de Nash des diagrammes des exposants initiaux est principale si et seulement si :

1) $\forall m \in \mathbb{N},(m, 0, \ldots, 0) \notin N_{i}^{l}$

2) $m_{i}^{l}=1$ 
On appelle $i^{\text {ieme }}$ partie principale de la stratification de $\mathcal{A}_{(X, x)}$ par les suites de Nash des diagrammes initiaux et on note $P_{(X, x)}^{i}$ la réunion (disjointe) des $S_{i, l}$ qui sont des strates principales.

Soient $m \in \mathbb{N}, \alpha=\left(\alpha_{0}, \alpha_{1}, \ldots, \alpha_{n}\right) \in \mathbb{N}^{n+1}$ tels que $|\alpha|=m$. On a $\alpha \leqslant(m, 0, \ldots, 0)$. Ainsi pour $f \in \mathbb{K}[[t, X]]$, dire que son exposant initial $\nu(f)$ est égal à $(m, 0, \ldots, 0)$ équivaut à dire que sa forme initiale $\operatorname{In}(f)$ vaut $a_{m} t^{m}$ avec $a_{m} \neq 0$. Par conséquent dire qu'un multi-indice $(m, 0 \ldots, 0)$ figure dans le diagramme des exposants initiaux de $I_{i, A^{2}}$ pour un point $A^{i}$ de $\mathbb{K}^{n i}$ équivaut à dire que $t^{m}$ appartient à l'idéal initial $\operatorname{In}\left(I_{i, A^{2}}\right)$ de $I_{i, A^{2}}$. Si $\mathbb{K}=\mathbb{C}$, l'existence d'un tel $m$ équivaut au fait que le réduit du cône tangent de $\left(Z_{i}^{\prime}, 0\right)$, noté $\left(C\left(Z_{i}^{\prime}, 0\right)\right)_{\text {red }}$, est inclus dans $\{t=0\}$ (cette dernière équation est celle du diviseur exeptionnel par la suite déclatements définie par $\left.A^{i}\right)$. La seconde condition correspond évidemment au fait que $\left(Z_{i}^{\prime}, 0\right)$ est lisse. Ces deux remarques montrent en particulier que lorsque $\mathbb{K}=\mathbb{C}$, $P_{(X, x)}^{i}$ est indépendant du système de coordonées choisi sur $\mathbb{K}^{n}$.

THÉORÈME 5.2. - Soit $(X, x)$ un germe de $\mathbb{K}$-espace analytique $\mathbb{K}$-réduit et equidimensionel de dimension $d$.

1) La suite $\left[P_{(X, x)}^{i}\right] \mathbb{L}^{-d i}$ converge vers une limite non nulle dans $\widehat{\mathcal{M}}_{\mathbb{K}}$.

2) La suite $\left[\Pi^{i}\left(\mathcal{A}_{(X, x)}\right)\right] \mathbb{L}^{-d i}-\left[P_{(X, x)}^{i}\right] \mathbb{L}^{-d i}$ converge vers zero dans $\widehat{\mathcal{M}}_{\mathbb{K}}$. Ainsi :

$$
\operatorname{Lim}_{i \rightarrow+\infty}\left[P_{(X, x)}^{i}\right] \mathbb{L}^{-d i}=\operatorname{Lim}_{i \rightarrow+\infty}\left[\Pi^{i}\left(\mathcal{A}_{(X, x)}\right)\right] \mathbb{L}^{-d i}
$$

Par conséquent :

$$
\operatorname{Lim}_{i \rightarrow+\infty}\left[P_{(X, x)}^{i}\right] \mathbb{L}^{-d i}=\mu\left(\mathcal{A}_{(X, x)}\right)
$$

où $\mu$ désigne la mesure motivique.

Preuve. - Nous commençons par constater que $P_{(X, x)}^{i} \subset \Pi^{i}\left(\mathcal{A}_{(X, x)}\right)$. En effet, soit $A^{i} \in P_{(X, x)}^{i}$. Par le second point de 5.1 le germe correspondant $\left(Z_{i}^{\prime}, 0\right) \subset\left(\mathbb{K}^{n+1}, 0\right)$ est lisse de dimension $d+1$. Par conséquent $N\left(I_{i, A^{2}}\right)=$ $\cup_{1 \leqslant k \leqslant n-d}\left(\alpha_{i_{k}}+\mathbb{N}^{n+1}\right)$ avec $\left|\alpha_{i_{k}}\right|=1$. Par la première condition de 5.1 $\alpha_{i_{k}} \neq(1,0, \ldots, 0), 1 \leqslant k \leqslant n-d$. Ceci implique que la base standard distinguée de $I_{i, A^{2}}$ est de la forme $X_{i_{k}}-U_{i_{k}}\left(t, X^{\prime \prime}\right), 1 \leqslant k \leqslant n-d$, où $X^{\prime \prime}$ désigne les $d$ coordonnées parmi $X_{1}, \ldots, X_{n}$, autres que $X_{i_{1}}, \ldots, X_{i_{n-d}}$. Par definition du diagramme $\left(D_{2}\right)$, ceci implique l'inclusion souhaitée. Montrons maintenant que la suite des $\left[P_{(X, x)}^{i}\right] \mathbb{L}^{-d i}$ est de Cauchy dans $\widehat{\mathcal{M}}_{\mathbb{K}}$. Soient $i, k \in \mathbb{N}^{*}$, par le second point de la Remarque 4.14 et la description ci-dessus, l'application :

$$
P_{(X, x)}^{i+k} \cap \Pi_{i+k, i}^{-1}\left(P_{(X, x)}^{i}\right) \longrightarrow P_{(X, x)}^{i}
$$


déduite de la projection oublie des « $k$ derniers termes» de $\mathbb{K}^{n(i+k)}$ dans $\mathbb{K}^{n i}$ est une fibration localement triviale de fibtre $\mathbb{L}^{d k}$. Ainsi $\left[P_{(X, x)}^{i+k} \cap\right.$ $\Pi_{i+k, i}^{-1}\left(P_{(X, x)}^{i}\right]=\left[P_{(X, x)}^{i}\right] \mathbb{L}^{d k}$ et donc :

$$
\begin{aligned}
{\left[P^{i+k}\right] \mathbb{L}^{-d(k+i)}-\left[P^{i}\right] \mathbb{L}^{-d i} } & =\left[P^{i+k}\right] \mathbb{L}^{-d(k+i)}-\left[P^{i+k} \cap \Pi_{i+k, i}^{-1}\left(P^{i}\right)\right] \mathbb{L}^{-d(i+k)} \\
& =\left[P^{i+k}-P^{i+k} \cap \Pi_{i+k, i}^{-1}\left(P^{i}\right)\right] \mathbb{L}^{-d(i+k)}
\end{aligned}
$$

$\grave{\mathrm{A}}$ un point $A^{i+k}=\left(A_{1}, \ldots, A_{i}, \ldots, A_{i+k}\right)$ de $P^{i+k}-P^{i+k} \cap \Pi_{i+k, i}^{-1}\left(P^{i}\right)$ correspond une suite des multiplicités de Nash $m_{0}, \ldots, m_{i}, \ldots, m_{i+k}$ avec $m_{i} \geqslant 2$ et $m_{i+k}=1$. Soit $p=n-d$ et $J_{p, 0}\left(\operatorname{resp} . J_{p, A^{2}}\right)$ l'idéal de $\mathbb{K}[[t, X]]$ engendré par $I_{0}$ et tous les déterminants mineurs d'ordre p des jacobiennes de $p$ éléments de $I_{0}$ (resp. engendré par $I_{i, A^{2}}$ et les déterminants mineurs d'ordre $p$ des jacobiennes de $p$ éléments de $\left.I_{i, A^{2}}\right)$. Soit $\varphi \in \mathcal{A}_{\left(\mathbb{K}^{n}, 0\right)}$ tel que $\varphi^{i}=A^{i}$. Puisque la suite des multiplicités de Nash est décroissante, on a $m_{l} \leqslant 2,0 \leqslant l \leqslant i$. Un calcul élémentaire montre alors que :

$$
\operatorname{ord}_{t}\left(\Gamma_{0}^{*}\left(J_{p, 0}\right)\right) \geqslant i+\operatorname{ord}_{t}\left(\Gamma_{i}^{*}\left(J_{p, A^{2}}\right)\right) \geqslant i+1 .
$$

Soit $\beta^{\prime}$ la fonction d'Artin-Greenberg de $\mathbb{K}[[t, X]] / J_{p, 0}$ et $c \geqslant 1$ tel que $\beta^{\prime}(i) \leqslant c i$. Notons $j=[i / c]$ la partie entière de $i / c$. Par définition de $\beta^{\prime}$, on a donc $A^{j} \in \Pi^{j}\left(\mathcal{A}_{(\operatorname{sing}(X), x)}\right)$. Il en découle que :

$d_{i+k}=\operatorname{dim}\left(P^{i+k}-P^{i+k} \cap \Pi_{i+k}^{-1}\left(P^{i}\right)\right) \leqslant(d-1) j+d(i+k-j)=d(i+k)-j$

Par suite $\operatorname{Lim}_{i \rightarrow+\infty} d(i+k)-d_{i+k}=+\infty$. Ce qui assure que la suite $\left[P^{i}\right] \mathbb{L}^{-d i}$ est de Cauchy dans $\widehat{\mathcal{M}}_{\mathbb{K}}$ et donc convergente.

Soit maintenant $A^{i} \in \Pi^{i}\left(\mathcal{A}_{(X, x)}\right)-P_{(X, x)}^{i}$. Soit $\varphi(t)=\sum_{k=1}^{i} A_{k} t^{k}+t^{i} \varphi_{i}(t)$ avec $\varphi_{i}(t)=\sum_{k=1}^{+\infty} A_{i+k} t^{k}$ un élément de $\mathcal{A}_{(X, x)}$ qui relève $A^{i}$. Par construction du diagramme $\left(D_{2}\right)$, l'arc $\Gamma_{i}(t)=\left(t, \varphi_{i}(t)\right)$ est tracé dans $\left(Z_{i}^{\prime}, 0\right)$. Ainsi le point $\left(1, A_{i+1}\right)$ est dans l'ensemble des zéros de l'idéal initial $\operatorname{In}\left(I_{i, A^{2}}\right)$ de $I_{i, A^{\imath}}$. Ceci implique que pour tout $m \in \mathbb{N},(m, 0, \ldots, 0) \notin N_{i, A^{2}}$. Par conséquent puisque $A^{i} \notin P^{i}$, la suite des multiplicités de Nash $\left(m_{0}, \ldots, m_{i}\right)$ de $A^{i}$ est telle que $m_{i} \geqslant 2$. Procédant comme plus haut en posant $j=[i / c]$, ceci implique que :

$$
\operatorname{ord}_{t}\left(\phi^{*} J_{p, 0}\right) \geqslant i+1, \forall \phi \in \mathcal{A}_{\left(\mathbb{K}^{n}, 0\right)} \mid \phi^{i}=A^{i}
$$

Par conséquent $A^{j} \in \Pi^{j}\left(\mathcal{A}_{(\operatorname{Sing}(X), x)}\right)$. Il en résulte que :

$$
l_{i}=\operatorname{dim}\left(\Pi^{i}\left(\mathcal{A}_{(X, x)}-P_{(X, x)}^{i}\right) \leqslant(d-1) j+d(i-j)=d i-j\right.
$$

Par suite $\operatorname{Lim}_{i \longrightarrow \infty} d i-l_{i}=+\infty$ et $\left[\Pi^{i}\left(\mathcal{A}_{(X, x)}\right)-P_{(X, x)}^{i}\right] \mathbb{L}^{-d i}$ converge vers zéro dans $\widehat{\mathcal{M}}_{\mathbb{K}}$. Il en découle la convergence des deux suites considérées et la 
coincidence de leurs limites. Le fait que cette limite soit égale à $\mu\left(\mathcal{A}_{(X, x)}\right)$ est conséquence de 7.1 p.229 dans [D-L1] (ou bien peut être considérer comme une définition du volume motivique c.f. [D-L2]).

Récemment $\mathrm{S}$. Ishii et J. Kollár ont considéré l'hypersurface de $\mathbb{C}^{5}$ définie par $X_{1}^{3}+X_{2}^{3}+X_{3}^{5}+X_{4}^{5}+X_{5}^{6}=0$ et montré qu'elle fournissait un contre exemple en dimension 4 au "probléme de Nash» (c.f. [N], [I.K], [R1], [R2] par exemple, pour la formulation de celui-ci). Nous nous proposons ici, pour illustrer ce qui précède et voir le rôle jouer par les suites de Nash, de calculer par les méthodes ci-dessus le volume motivique de telles hypersurfaces. On peut bien entendu faire le calcul de manière indépendante en déterminant une résolution des singularités et utiliser «les formules de changement de variables» de l'intégration motivique de [D-L1] ou [S].

Exemple $5.3 .-\mathbb{K}=\mathbb{C}$. Soient $n \geqslant 3, k \geqslant 2$ et $\left(H_{n, k}, 0\right)$ le germe d'hypersurface à l'origine de $\mathbb{K}^{n+1}$ défini par :

$$
X_{1}^{k}+\ldots+X_{n}^{k}+Y^{2 k}=0,
$$

(Le contre-exemple d'Ishii-Kollár est le cas $n=4, k=3$ )

Posons pour $p \geqslant 1, V_{p, k}=\left\{\left(X_{1}, \ldots, X_{p}\right) \in \mathbb{K}^{p} / 1+X_{1}^{k}+\ldots+X_{p}^{k}=0\right\}$.

On a alors :

$$
\begin{aligned}
\mu\left(\mathcal{A}_{\left(H_{n, k}, 0\right)}\right) & =\left(\left[V_{n-1, k}\right]+\left[V_{n-2, k}\right]+\ldots+k\right) \mathbb{L} \frac{\mathbb{L}-1}{\mathbb{L}^{n}-1}+\left[V_{n, k}\right] \frac{\mathbb{L}-1}{\mathbb{L}^{2 n-1}-1} \\
& +\left(\left[V_{n-1, k}\right]+\left[V_{n-2, k}\right]+\ldots+k\right) \frac{(\mathbb{L}-1)^{2}}{\left(\mathbb{L}^{n-1}-1\right)\left(\mathbb{L}^{2 n-1}-1\right)}
\end{aligned}
$$

Nous indiquons brièvement les principales étapes des calculs. Pour cela, désignons par $C_{k}$ et $W_{k}$ les constructibles :

$$
\begin{array}{r}
C_{k}=\left\{X \in \mathbb{K}^{n} / X \neq 0 \text { et } X_{1}^{k}+\ldots+X_{n}^{k}=0\right\} \\
W_{k}=\left\{(X, Y) \in \mathbb{K}^{n+1} / Y \neq 0 \text { et } X_{1}^{k}+\ldots+X_{n}^{k}+Y^{2 k}=0\right\} .
\end{array}
$$

Il est élémentaire de vérifier que dans $K_{0}\left(\mathcal{V}_{\mathbb{K}}\right)$ on a :

$$
\left[C_{k}\right]=\left(\left[V_{n-1, k}\right]+\left[V_{n-2, k}\right]+\ldots+k\right)(\mathbb{L}-1) \text { et }\left[W_{k}\right]=\left[V_{n, k}\right](\mathbb{L}-1) .
$$

Soit maintenant $\varphi^{i}=A^{i}=\left(A_{1}, \ldots, A_{i}\right) \in \mathbb{K}^{(n+1) i}, A_{l}=\left(B_{l}, Y_{l}\right) \in \mathbb{K}^{n+1}$ avec $B_{l}=\left(b_{l, 1}, \ldots, b_{l, n}\right)$. Posons $v=\operatorname{Min}\left\{l / A_{l} \neq 0\right\}$. Si $A^{i} \in P^{i}$ alors on a $v \leqslant i$, sans quoi $m_{i}=k$. L'équation locale de $\left(Z_{v}^{\prime}, 0\right)$ est :

$$
\left(X_{1}+b_{v, 1}\right)^{k}+\ldots+\left(X_{n}+b_{v, n}\right)^{k}+t^{k v}\left(Y+Y_{v}\right)^{2 k}=0 .
$$


Deux éventualités peuvent se présenter :

- $B_{v} \neq$ et alors $m_{v}>0$ si et seulement si $B_{v} \in C_{k}$. On alors $m_{v}=1$. Ainsi les éléments $A^{i}$ de $P^{i}$ au dessus de tels points sont une fibration localement triviale de fibre $\mathbb{L}^{n(i-v)}$ et de base $C_{k} \times \mathbb{L}$. Ainsi leur contribution à $\left[P^{i}\right] \mathbb{L}^{-n i}$ est $\left[C_{k}\right] \mathbb{L} . \mathbb{L}^{-n v}$. Faisant la somme pour $v$ variant de 1 à $i$, et prenant la limite dans $\widehat{\mathcal{M}}_{\mathbb{K}}$ lorsque $i$ tend vers $+\infty$ on obtient :

$$
\left[C_{k}\right] \mathbb{L} \times \frac{1}{\mathbb{L}^{n}-1}=\left(\left[V_{n-1, k}\right]+\left[V_{n-2, k}\right]+\ldots+k\right) \mathbb{L} \frac{\mathbb{L}-1}{\mathbb{L}^{n}-1}
$$

Ceci est la contribution des strates principales correspondant au suites de Nash $(k, \ldots, k, 1, \ldots, 1)$, le premier 1 en position $v$ et $\operatorname{ord}_{t}\left(\varphi^{i}\right)=v$. Le découpage de $\left[C_{k}\right]$ en $\left[V_{n-1, k}\right]+\left[V_{n-2, k}\right]+\ldots+k$ correspond aux différentes possibilités pour $N^{v}=\alpha+\mathbb{N}^{n+1}$, avec $\alpha=(0, \beta)$ et $|\beta|=1$.

- $B_{v}=0$ et donc $Y_{v} \neq 0$. On considère le premier $l$ avec $v+l \leqslant i$ tel que $B_{v+l} \neq 0$. Si $A^{i} \in P^{i}$, on a certainement $l \leqslant v$. Sinon l'équation locale de $\left(Z_{2 v}^{\prime}, 0\right)$ est :

$$
X_{1}^{k}+\ldots+X_{n}^{k}+\left(t^{v} Y+t^{v} Y_{2 v}+\ldots+Y_{v}\right)^{2 k}=0
$$

et l'on a $m_{2 v}=0$. On a alors deux sous-cas.

- a) $l=v$. L'équation locale de $\left(Z_{2 v}^{\prime}, 0\right)$ est alors :

$$
\left(X_{1}+b_{2 v, 1}\right)^{k}+\ldots+\left(X_{1}+b_{2 v, 1}\right)^{k}+\left(t^{v} Y+t^{v} Y_{2 v}+\ldots+Y_{v}\right)^{2 k}=0 .
$$

On a $m_{2 v}>0$ si et seulement si $\left(B_{2 v}, Y_{v}\right) \in W_{k}$ et alors $m_{2 v}=1$. Les éléments de $P^{i}$ au dessus de tels points sont une fibration localement triviale de fibre $\mathbb{L}^{n(i-2 v)}$ et de base $W_{k} \times \mathbb{L}^{v}$. Leur contribution a $\left[P^{i}\right] \mathbb{L}^{-n i}$ est donc $\left[W_{k}\right] \mathbb{L}^{-(2 n-1) v}$. Prenant la somme pour $v$ variant de 1 à $[i / 2]$ et passant à la limite on obtient :

$$
\left[W_{k}\right] \times \frac{1}{\mathbb{L}^{2 n-1}-1}=\left[V_{n, k}\right] \frac{\mathbb{L}-1}{\mathbb{L}^{2 n-1}-1}
$$

Ces points correspondent aux suites de $\operatorname{Nash}(k, \ldots, k, 1, \ldots, 1)$, le premier 1 en position $2 v$ et $\operatorname{ord}_{t}\left(\varphi^{i}\right)=v$.

$-b) 1 \leqslant l<v$ et $l+v \leqslant i$. On a en particulier $v \geqslant 2$. L'équation locale de $\left(Z_{v+l}^{\prime}, 0\right)$ est

$$
\left(X_{1}+b_{1, v+l}\right)^{k}+\ldots+\left(X_{n}+b_{n, v+l}\right)^{k}+t^{k(v-l)}\left(t^{l} Y+t^{l} Y_{l+v}+\ldots+Y_{v}\right)^{2 k}=0
$$

On alors $m_{v+l}>0$ si et seulement si $B_{v+l} \in C_{k}$ et alors $m_{v+l}=1$. Ainsi les éléments de $P^{i}$ au dessus de tels points sont une fibration localement triviale de fibre $\mathbb{L}^{n(i-v-l)}$ et de base $(\mathbb{L}-1)\left[C_{k}\right] \mathbb{L}^{l}$. Ainsi la contribution 
à $\left[P^{i}\right] \mathbb{L}^{-n i}$ est $(\mathbb{L}-1)\left[C_{k}\right] \mathbb{L}^{-n(v+l)} \mathbb{L}^{l}$. Faisant varier $v$ de 2 à $i$ et $l$ de 1 à $i_{v}=\operatorname{Min}(v-1, i-v)$, on obtient :

$$
\left[C_{k}\right](\mathbb{L}-1) \sum_{v=2}^{i} \mathbb{L}^{-n v} \sum_{l=1}^{i_{v}} \mathbb{L}^{-(n-1) l}=\left[C_{k}\right](\mathbb{L}-1) \sum_{v=2}^{i} \mathbb{L}^{-n v} \frac{1-\mathbb{L}^{-(n-1) i_{v}}}{\mathbb{L}^{n-1}-1}
$$

Posons $j=[(i+1) / 2]$. Nous obtenons :

$$
\left[C_{k}\right](\mathbb{L}-1) \frac{1}{\mathbb{L}^{n-1}-1}\left(\sum_{v=2}^{i} \mathbb{L}^{-n v}-\mathbb{L}^{n-1} \sum_{v=2}^{j} \mathbb{L}^{-(2 n-1) v}-\sum_{v=j+1}^{i} \mathbb{L}^{-(n-1) i} \mathbb{L}^{-v}\right)
$$

La troisième somme est de limite nulle. La limite de la différence des deux premières sommes vaut :

$$
\frac{\mathbb{L}^{n-1}-1}{\left(\mathbb{L}^{n}-1\right)\left(\mathbb{L}^{2 n-1}-1\right)}
$$

Il vient donc (après simplification par $\mathbb{L}^{n-1}-1$ ) :

$$
\left[C_{k}\right] \frac{\mathbb{L}-1}{\left(\mathbb{L}^{n}-1\right)\left(\mathbb{L}^{2 n-1}-1\right)}=\left(\left[V_{n-1, k}\right]+\left[V_{n-2, k}\right]+\ldots+k\right) \frac{(\mathbb{L}-1)^{2}}{\left(\mathbb{L}^{n}-1\right)\left(\mathbb{L}^{2 n-1}-1\right)}
$$

\section{Condition de rang de Gabrielov et espace des arcs}

Dans cette section, $\mathbb{K}=\mathbb{C}$. Soit $F_{x}:(X, x) \rightarrow(Y, y)$ un germe de morphisme entre deux germes irréductibles. Notons $\mathcal{F}_{x}: \mathcal{A}_{(X, x)} \rightarrow \mathcal{A}_{(Y, y)}$ le morphisme induit. Rappelons que nous désignons par rang générique de $F_{x}$ le nombre :

$$
r_{1}\left(F_{x}\right)=\operatorname{grk}\left(F_{x}\right)=\operatorname{In} f_{U}\left(\operatorname{Sup} x_{x^{\prime}} \operatorname{Rang}\left(F_{x^{\prime}}\right)\right)
$$

où $x^{\prime} \in \operatorname{Reg}(X \cap U)$ et $U$ parcourt une base de voisinage $x$. Nous cherchons à établir :

THÉORÈME 6.1. - Les propriétés suivantes sont équivalentes :

1) $\operatorname{grk}\left(F_{x}\right)=\operatorname{dim}_{y} Y$;

2) Pour tout ouvert $\mathcal{V}$ de $\mathcal{R}_{(X, x)}, \mathcal{F}_{x}(\mathcal{V})$ contient un ouvert de $\mathcal{R}_{(Y, y)}$;

3) $\mathcal{F}\left(\mathcal{R}_{(X, x)}\right)$ contient un ouvert de $\mathcal{R}_{(Y, y)}$. 


\section{Preuve.-}

1) $\Rightarrow 2$ )

Soient $\varphi \in \mathcal{R}_{(X, x)}$ et $i \in \mathbb{N}$, nous allons prouver que : $\mathcal{F}_{x}\left(B_{i}(\varphi)\right)$ contient un ouvert de $\mathcal{R}_{(Y, y)}$. Nous commençons d'abord par nous ramener au cas où $(X, x)$ est lisse.

$\underline{\text { Réduction au cas où }(X, x) \text { est lisse : }}$

Considérons un plongement $(X, x) \rightarrow\left(\mathbb{K}^{m}, 0\right)$. Soient $I \subset \mathbb{K}\left\{x_{1}, \ldots, x_{m}\right\}$ l'idéal définissant $(X, x), l=\operatorname{haut}(I)$. Notons $J_{l}$ l'idéal engendré par $I$ et tous les déterminants jacobiens d'ordre $l$ des $l$-upplets d'éléments de $I$. Puisque $\varphi \in \mathcal{R}_{(X, x)}, \operatorname{ord}\left(\varphi^{*} J_{l}\right)=u<+\infty$. Par le procédé utilisé au Théorème 4.1, on peut trouver un germe $(Z, z)$ lisse, un germe de morphisme $: \Pi_{z}:(Z, z) \rightarrow(X, x)$ obtenu par une succession de $u$ éclatements ponctuels et $\phi \in \mathcal{A}_{(Z, z)}$ qui relève $\varphi$ à travers $\Pi_{z}$. Soit alors $G_{z}:(Z, z) \underset{\rightarrow}{\Pi_{z}}(X, x) \underset{\rightarrow}{\rightarrow}(Y, y)$. Il est alors élémentaire de vérifier que $\operatorname{grk}\left(G_{z}\right)=\operatorname{grk}\left(F_{x}\right)$. Si l'assertion 2) est prouvée pour $\mathcal{G}_{z}$, elle le sera pour $\mathcal{F}_{x}$. En effet, soit $i \in \mathbb{N}$, s'il existe $j \in \mathbb{N}$ et $\theta \in \mathcal{R}_{(Y, y)}$ telle que :

$$
B_{j}(\theta) \subset \mathcal{G}_{z}\left(B_{i}(\phi)\right)
$$

on aura :

$$
B_{j}(\theta) \subset \mathcal{G}_{z}\left(B_{i}(\phi)\right)=\mathcal{F}_{x}\left(\Pi_{z}\left(B_{i}(\phi)\right) \subset \mathcal{F}_{x}\left(B_{i}(\varphi)\right) .\right.
$$

Nous supposerons donc désormais que $(X, x)=\left(\mathbb{K}^{m}, 0\right)$. Soit alors $d=$ $\operatorname{dim}_{y} Y$. Plongeons $(Y, y)$ dans $\left(\mathbb{K}^{n}, 0\right)$. Nous noterons $F_{1}\left(x_{1}, \ldots, x_{m}\right), \ldots$, $F_{n}\left(x_{1}, \ldots, x_{m}\right)$ les composantes de $F_{x}$, et par $F$ un représentant de $F_{x}$. Pour un choix générique de coordonnées linéaires à l'origine de $\left(\mathbb{K}^{n}, 0\right)$, on peut supposer être dans la situation qui suit.

Soit $P_{0}:(Y, 0) \rightarrow\left(\mathbb{K}^{d}, 0\right)$, le germe de morphisme induit par la projection :

$$
\begin{aligned}
\mathbb{K}^{n} & \rightarrow \mathbb{K}^{d} \\
\left(z_{1}, \ldots, z_{n}\right) & \rightarrow\left(z_{1}, \ldots, z_{d}\right)
\end{aligned}
$$

Le morphisme :

$$
P_{0}^{*}: \mathcal{O}_{d}=\mathbb{K}\left\{z_{1}, \ldots, z_{d}\right\} \longrightarrow \mathcal{O}_{Y, 0}=\mathcal{O}_{n} / J
$$

est injectif et fini. 
Si $k$ est la dimension du corps de fractions de $\mathcal{O}_{Y, 0}$ sur le corps des fractions de $\mathcal{O}_{d}$, le polynôme minimal de $\bar{z}_{d+1}$ est un polynôme distingué, $P(Z)=Z^{k}+\sum_{l=1}^{k} a_{l} Z^{k-l}$ à coefficients dans $\mathcal{O}_{d}$.

Le discriminant $\Delta\left(z_{1}, \ldots, z_{d}\right)$ de ce polynôme est non nul et :

$$
\bar{\Delta} . \mathcal{O}_{Y, 0} \subset \mathcal{O}_{d}+\mathcal{O}_{d} \cdot \bar{z}_{d+1}+\ldots+\mathcal{O}_{d} \cdot \bar{z}_{d+1}^{k-1}
$$

En particulier, $z_{d+1}^{k}+\sum_{l=1}^{k} a_{l} z_{d+1}^{k-l} \in J$, et pour $2 \leqslant j \leqslant n-d$, il existe des $a_{l, j} \in \mathcal{O}_{d}$ tels que :

$$
\Delta z_{d+j}-\sum_{l=1}^{k-1} a_{l, j} z_{d+1}^{l} \in J .
$$

D'autre part, le choix de la projection linéaire $P_{0}$ étant générique et $\operatorname{grk}\left(F_{x}\right)=$ $d$, on peut supposer quitte à renuméroter les coordonnées dans $\mathbb{K}^{m}$ que :

$$
J_{1}\left(x_{1}, \ldots, x_{m}\right)=\operatorname{det}\left(\frac{\partial F_{l}}{\partial x_{j}}\left(x_{1}, \ldots, x_{m}\right)\right)_{1 \leqslant l \leqslant d, 1 \leqslant j \leqslant d}
$$

est non identiquement nul.

Notons $(D, 0) \subset\left(\mathbb{K}^{d}, 0\right)$ le germe en 0 des zéros de $\Delta$. On a $\Delta \circ F_{x} \neq$ 0 , sans quoi $F_{x}\left(\left(\mathbb{K}^{m}, 0\right)\right) \subset P_{0}^{-1}(D, 0)$ d'où $\operatorname{grk}\left(F_{x}\right) \leqslant \operatorname{dim}_{0} P_{0}^{-1}(D, 0)=$ $d-1<d$.

De la même façon $(V, 0)=F_{x}^{-1}(\operatorname{Sing}(Y), 0) \subset\left(\mathbb{K}^{m}, 0\right)$ est un sousensemble analytique propre de $\left(\mathbb{K}^{m}, 0\right)$. Soit $I_{V}$ l'idéal réduit définissant $(V, 0)$ et $h \in I_{V}, h \neq 0$. Posons $k_{1}=m_{0}(h), k_{2}=m_{0}\left(J_{1}\right), k_{3}=m_{0}\left(\Delta \circ F_{x}\right)$, où $m_{0}()$ désigne la multiplicité ou l'ordre à l'origine.

Soient $\phi \in \mathcal{A}_{\left(\mathbb{K}^{m}, 0\right)}$ et $i \in \mathbb{N}$, en vertu du Lemme 4.9 , il existe $\alpha \in B_{i}(\phi)$ telle que :

$$
\operatorname{ord}\left(h(\alpha(t)) . J_{1}(\alpha(t)) \cdot \Delta(F(\alpha(t))) \leqslant\left(k_{1}+k_{2}+k_{3}\right)(i+1)=s .\right.
$$

Posons $\theta(t)=F(\alpha(t))$. Nous allons voir que :

$$
\begin{aligned}
& -B_{2 s+1}(\theta) \subset \mathcal{F}_{x}\left(B_{i}(\phi)\right)=\mathcal{F}_{x}\left(B_{i}(\alpha)\right) \\
& -B_{2 s+1}(\theta) \subset \mathcal{R}_{(Y, 0)} .
\end{aligned}
$$


Soit $\theta^{\prime}=\left(\theta_{1}^{\prime}, \ldots, \theta_{d}^{\prime}, \theta_{d+1}^{\prime}, \ldots, \theta_{n}^{\prime}\right) \in B_{2 s+1}(\theta) \subset \mathcal{A}_{(Y, 0)}$. Considérons le système d'équations implicites :

$$
\begin{gathered}
G_{1}\left(t, y_{1}, \ldots, y_{m}\right)=F_{1}\left(\alpha_{1}(t)+y_{1}, \ldots, \alpha_{m}(t)+y_{m}\right)-\theta_{1}^{\prime}(t)=0 \\
\vdots \\
G_{d}\left(t, y_{1}, \ldots, y_{m}\right)=F_{d}\left(\alpha_{1}(t)+y_{1}, \ldots, \alpha_{m}(t)+y_{m}\right)-\theta_{d}^{\prime}(t)=0 .
\end{gathered}
$$

Soit $I$ l'idéal de $\mathcal{O}_{1}$ engendré par les mineurs d'ordre $d$ de la jacobienne $G_{y}^{\prime}(t, 0)$. On a $J_{1}(\alpha(t)) \in I$. Soit $l=\operatorname{ord}(I) \leqslant \operatorname{ord}\left(J_{1}(\alpha(t)) \leqslant s\right.$.

Par hypothèse, $G(t, 0) \in(t)^{2 s+1} \cdot \mathcal{O}_{1}^{d}$. Donc $G(t, 0) \in \oplus_{d}(t)^{2(s-l)+1} \cdot I^{2}$. Par le théorème des fonctions implicites de J.C. Tougeron, il existe $y_{1}(t), \ldots$, $y_{m}(t) \in(t)^{2(s-l)+1} .(t)^{l} \subset(t)^{s+1}$ telles que :

$$
F_{j}(\alpha(t)+y(t))=\theta_{j}^{\prime}(t), 1 \leqslant j \leqslant d .
$$

Posons $\rho(t)=\alpha(t)+y(t)$. On a donc $\rho \in B_{s}(\alpha) \subset B_{i}(\alpha)$.

Pour conclure, il nous reste à voir que pour $d+1 \leqslant j \leqslant n$, on a : $F_{j}(\rho(t))=\theta_{j}^{\prime}(t)$.

Pour cela remarquons que, puisque $\theta^{\prime} \in B_{2 s+1}(\theta) \subset \mathcal{A}_{(Y, 0)}, \Delta\left(\theta^{\prime}\right)$ et $\Delta(\theta)$ coincïdent jusqu'à l'ordre $2 s$. Mais $\operatorname{ord}(\Delta(\theta)) \leqslant s$, donc $\operatorname{ord}\left(\Delta\left(\theta^{\prime}\right)\right) \leqslant s$.

Posons : $\tilde{\theta}^{\prime}(t)=\left(\theta_{1}^{\prime}(t), \ldots, \theta_{d}^{\prime}(t)\right)$. Maintenant, puisque $F$ est à valeur dans $(Y, 0)$ et $\left(F_{1}(\rho(t)), \ldots, F_{d}(\rho(t))\right)=\tilde{\theta}^{\prime}(t)$.

On a :

$$
\begin{gathered}
F_{d+1}(\rho(t))^{k}+\sum_{l=1}^{k} a_{l}\left(\tilde{\theta}^{\prime}(t)\right) F_{d+1}^{k-l}(\rho(t))=0 \\
\theta_{d+1}^{\prime}(t)^{k}+\sum_{l=1}^{k} a_{l}\left(\tilde{\theta}^{\prime}(t)\right)\left(\theta_{d+1}^{\prime}\right)^{k-l}(t)=0
\end{gathered}
$$

Par suite $F_{d+1}(\rho(t))$ et $\theta_{d+1}^{\prime}(t)$ sont donc deux racines du polynôme $P(U)=$ $U^{k}+\sum_{l=1}^{k} a_{l}\left(\tilde{\theta}^{\prime}(t)\right) U^{k-l}$. D'après le théorème de Puiseux, il existe, $u_{1}\left(t^{1 / k !}\right)$, $\ldots, u_{k}\left(t^{1 / k !}\right)$ analytiques complexes en $t^{1 / k !}$ solutions de $P(U)=0$. Supposons que $F_{d+1}(\rho(t)) \neq \theta_{d+1}^{\prime}(t)$, alors :

$$
\left(F_{d+1}\left(\rho\left(t^{k !}\right)\right)-\theta_{d+1}^{\prime}\left(t^{k !}\right)\right)^{2} \text { divise } \prod_{l<j}\left(u_{l}(t)-u_{j}(t)\right)^{2}=\Delta\left(\tilde{\theta}^{\prime}\left(t^{k !}\right)\right) .
$$


Par suite :

$$
\operatorname{ord}\left(F_{d+1}(\rho(t))-\theta_{d+1}^{\prime}(t)\right) k ! \leqslant \operatorname{ord}\left(\Delta\left(\tilde{\theta}^{\prime}(t)\right) k ! \leqslant s k ! .\right.
$$

Or ceci est absurde car :

$\operatorname{ord}\left(F_{d+1}(\rho(t))-F_{d+1}(\alpha(t)) \geqslant s+1\right.$ et $\operatorname{ord}\left(\theta_{d+1}^{\prime}(t)-F_{d+1}(\alpha(t))\right) \geqslant 2 s+1$.

Par suite $F_{d+1}(\rho(t))=\theta_{d+1}^{\prime}(t)$. Ensuite, pour $j \geqslant 2$, on a :

$$
\begin{aligned}
& \Delta\left(\tilde{\theta}^{\prime}(t)\right) F_{d+j}(\rho(t))=\sum_{l=0}^{k-1} a_{l, j}\left(\tilde{\theta}^{\prime}(t)\right) F_{d+1}^{l}(\rho(t)) \\
& =\sum_{l=0}^{k-1} a_{l, j}\left(\tilde{\theta}^{\prime}(t)\right)\left(\theta_{d+1}^{\prime}\right)^{l}(t)=\Delta\left(\tilde{\theta}^{\prime}(t)\right) \theta_{d+j}^{\prime}(t) .
\end{aligned}
$$

Par suite, $F_{d+j}(\rho(t))=\theta_{d+j}^{\prime}(t), j \geqslant 2$. Donc $\rho$ relève $\theta^{\prime}$ par $F$. Enfin notons que $h(\rho(t)) \neq 0$ car $\operatorname{ord}(h(\rho(t))-h(\alpha(t))) \geqslant s+1$ et $\operatorname{ord}(h(\alpha(t)) \leqslant s$. Par conséquent, $\rho(t)$ n'est pas tracée dans $F^{-1}(\operatorname{Sing}(Y), 0)$. Donc à fortiori $F(\rho(t))=\theta^{\prime}(t)$ n'est pas tracée dans $(\operatorname{Sing}(Y), 0)$.

Nous prouvons à présent l'implication 3$) \Rightarrow 1$ ).

Considérons $F_{x}:(X, 0) \rightarrow(Y, 0)$ tel que $\mathcal{F}\left(\mathcal{R}_{(X, 0)}\right)$ contienne un ouvert de $\mathcal{R}_{(Y, 0)}$. Posons $d=\operatorname{dim}_{0} Y$ et $P_{0}:(Y, 0) \rightarrow\left(\mathbb{K}^{d}, 0\right)$ une projection linéaire générique. D'après l'implication 1) $\Rightarrow 2$ ), puisque $\operatorname{grk}\left(P_{0}\right)=d$, pour tout ouvert $\mathcal{V}$ de $\mathcal{R}_{(Y, 0)}, \mathcal{P}_{0}(\mathcal{V})$ contient un ouvert de $\mathcal{A}_{\left(\mathbb{K}^{d}, 0\right)}$. Par suite, posant $F^{\prime}=P_{0} \circ F, \mathcal{F}^{\prime}\left(\mathcal{R}_{(X, 0)}\right)$ contient un ouvert de $\mathcal{A}_{\left(\mathbb{K}^{d}, 0\right)}$. Mais $\operatorname{grk}\left(F^{\prime}\right) \leqslant g r k(F)$. L'implication 3$) \Rightarrow 1$ ) sera donc prouvée si l'on montre que $\operatorname{grk}\left(F^{\prime}\right)=d$. Autrement dit, on peut supposer que notre germe d'application $F:(X, 0) \rightarrow\left(\mathbb{K}^{d}, 0\right)$ est telle que $\mathcal{F}\left(\mathcal{R}_{(X, 0)}\right)$ contient un ouvert de $\mathcal{A}_{\left(\mathbb{K}^{d}, 0\right)}$.

Considérons une boule de rayon $r$ suffisamment petit, $B(0, r) \subset \mathbb{K}^{m}$ pour que :

$$
X \cap B(0, r)=\left\{z \in B(0, r) / f_{1}(z)=\ldots=f_{p}(z)=0\right\},
$$

avec $f_{1}, \ldots, f_{p}$ analytiques sur $B(0, r)$.

$$
\begin{gathered}
\operatorname{Sing}(X) \cap B(0, r)=\left\{z \in B(0, r) / f_{1}(z)=\ldots=f_{p}(z)=0,\right. \\
\left.\operatorname{det}\left(\frac{\partial f_{i_{l}}}{\partial z_{j_{k}}}\right)_{1 \leqslant l \leqslant s, 1 \leqslant k \leqslant s}(z)=0, \forall \underline{i}=\left(i_{1}, \ldots, i_{s}\right), \underline{j}=\left(j_{1}, \ldots, j_{s}\right)\right\}
\end{gathered}
$$

où $m-s=\operatorname{dim}(X, 0)$. 
Soit $r_{n}$ une suite croissante telle que $0<r_{n}<r$ et $r_{n} \rightarrow r$. Posons :

$$
R_{n, l}=\left\{z \in \mathbb{K}^{m} /|z| \leqslant r_{n}, z \in X \cap B(0, r), \sum_{\underline{i}, \underline{j}}\left|\operatorname{det}\left(\frac{\partial f_{i_{j}}}{\partial z_{j_{k}}}\right)_{1 \leqslant j, k \leqslant s}(z)\right| \geqslant \frac{1}{l}\right\} .
$$

On a évidemment : $\operatorname{Reg}(X) \cap B(0, r)=\cup_{l, n \in \mathbb{N}^{*}} R_{n, l}$. Par suite $F(\operatorname{Reg}(X) \cap B(0, r))=\cup_{n, l} F\left(R_{n, l}\right)$ est réunion dénombrable de compacts de $\mathbb{K}^{d}$. Donc l'ensemble $F(\operatorname{Reg}(X) \cap B(0, r))$ est mesurable pour la mesure de Lebesgue sur $\mathbb{K}^{d}$. Supposons $g r k(F)<d$. Écrivons $\operatorname{Re} g(X) \cap B(0, r)=$ $\cup_{n \in \mathbb{N}} D_{n}$ où chaque $D_{n}$ est un domaine de carte. Posons $F_{n}=F / D_{n}$. En tout point de $D_{n}$ le rang de la jacobienne complexe de $F_{n}$ est strictement inférieur à $d$. Donc le rang de la jacobienne réelle de $F_{n}$ est strictement inférieur à $2 d$. Tout point de $F_{n}\left(D_{n}\right)$ est donc valeur critique réelle de $F_{n}$. Par le théorème de Sard, $F_{n}\left(D_{n}\right)$ est donc de mesure nulle. Il en est donc de même de $F(\operatorname{Reg}(X) \cap B(0, r))=\cup F_{n}\left(D_{n}\right)$. Nous allons voir que ceci est contradictoire avec le fait que $\mathcal{F}\left(\mathcal{R}_{(X, 0)}\right)$ contienne un ouvert de $\mathcal{A}_{\left(\mathbb{K}^{d}, 0\right)}$.

Soient $\varphi \in \mathcal{A}_{\left(\mathbb{K}^{d}, 0\right)}$ et $i \in \mathbb{N}$ tel que $B_{i}(\varphi) \subset \mathcal{F}\left(\mathcal{R}_{(X, 0)}\right)$. Posons $\varphi(t)=$ $\sum_{k=1}^{+\infty} A_{k} t^{k}, A_{k}=\left(a_{k}^{1}, \ldots, a_{k}^{d}\right) \in \mathbb{K}^{d}$. Considérons alors l'application :

$$
\begin{gathered}
A: \mathbb{K} \times \mathbb{K}^{d} \longrightarrow \mathbb{K}^{d} \\
(t, x) \longrightarrow A(t, x)=\sum_{k=1}^{i} A_{k} t^{k}+x . t^{i+1} .
\end{gathered}
$$

$F(\operatorname{Reg}(X) \cap B(0, r))$ est réunion dénombrable de compacts, donc de fermés. Il en est donc de même de $V=A^{-1}(F(\operatorname{Reg}(X) \cap B(0, r)))$. Soient $l \geqslant 1$ et $C_{l}=\{z \in \mathbb{K} /|z|=1 / l\}$ le cercle de centre l'origine et de rayon $1 / l$. Considérons :

$$
V_{l}=V \cap\left(C_{l} \times \mathbb{K}^{d}\right) .
$$

$V_{l}$ est donc réunion dénombrable de compacts. Soit $X_{l}=P\left(V_{l}\right)$, où $P: \mathbb{K} \times$ $\mathbb{K}^{d} \rightarrow \mathbb{K}^{d}$ est la projection canonique. $X_{l}$ est donc mesurable. Maintenant, le fait que $B_{i}(\varphi) \subset \mathcal{F}\left(\mathcal{R}_{(X, 0)}\right)$ implique que :

$$
\mathbb{K}^{d}=\cup_{l \geqslant 1} X_{l}
$$

En effet, soit $x \in \mathbb{K}^{d}$, l'arc $t \rightarrow \alpha(t)=A(t, x)$ est un élément de $B_{i}(\varphi)$. Il se relève donc par $F$ en un arc $\rho \in \mathcal{R}_{(X, 0)}$. Donc pour $|t|=1 / l$ assez petit, $\alpha(t)=A(t, x) \in F\left(\operatorname{Reg}(X \cap B(0, r))\right.$ et donc $x \in X_{l}$. Maintenant par $(*)$, l'un des $X_{l}$, disons $X_{l_{0}}$ est de mesure non nulle. Il en découle que l'ensemble :

$$
\begin{gathered}
Z_{l_{0}}=\left\{z \in \mathbb{K}^{d} / z=\sum_{k=1}^{i} A_{k}\left(\frac{1}{l_{0}}\right)^{k}+u\left(\frac{1}{l_{0}}\right)^{i+1}, u \in X_{l_{0}}\right\} \\
-46-
\end{gathered}
$$


est de mesure non nulle. Mais par définition, $Z_{l_{0}} \subset F(\operatorname{Reg}(X) \cap B(0, r))$. Ce dernier ne peut donc être de mesure nulle.

Nous terminons cette section par quelques remarques et commentaires. Pour cela si $(X, x)$ est un germe d'espace analytique, nous noterons par $\widehat{\mathcal{O}}_{X, x}$ le complété $\mathcal{M}_{X, x}$ addique de $\mathcal{O}_{X, x}$. De la même façon si $F_{x}:(X, x) \rightarrow(Y, y)$ est un germe de morphisme, on notera $\widehat{F}_{x}^{*}: \widehat{\mathcal{O}}_{Y, y} \rightarrow \widehat{\mathcal{O}}_{X, x}$ le morphisme induit par $F_{x}$. On a alors le corollaire suivant qui est un cas particulier des théorèmes de A.M. Gabrielov et S. Izumi (cf. [I4]).

Corollaire 6.2. - Soit $(X, x)$ un germe d'espace réduit et $F_{x}:(X, x) \rightarrow$ $\left(\mathbb{C}^{d}, 0\right)$ tel que grk $\left(F_{x}\right)=d$. Alors :

1) Si $S \in \widehat{\mathcal{O}}_{d}$ est tel que ${\widehat{F_{x}}}^{*}(S) \in \mathcal{O}_{X, x}$, alors $S \in \mathcal{O}_{d}$;

2) $\exists a, b \in \mathbb{R}^{+} / \forall S \in \widehat{\mathcal{O}}_{d}, m\left(\widehat{F}_{x}^{*}(S)\right) \leqslant a m(S)+b$ où " $m$ " désigne la multiplicité ou l'ordre.

Preuve. - Puisque le rang générique de $F_{x}$ est le maximum des rangs génériques des restrictions de $F_{x}$ aux composantes irréductibles de $(X, x)$, on peut supposer que celui-ci est irréductible. Mais alors d'après le Théorème 1.4, il existe $\varphi \in \mathcal{A}_{\left(\mathbb{C}^{d}, 0\right)}$ et $i \in \mathbb{N}$ tels que $B_{i}(\varphi) \subset \mathcal{F}_{x}\left(\mathcal{R}_{(X, x)}\right) \subset \mathcal{F}_{x}\left(\mathcal{A}_{(X, x)}\right)$. Soit alors $S \in \widehat{\mathcal{O}}_{d}$, tel que $\widehat{F}_{x}^{*}(S) \in \mathcal{O}_{(X, x)}$. Par suite, pour tout $\theta \in \mathcal{A}_{(X, x)}$, ${\widehat{F_{x}}}^{*}(S)(\theta) \in \mathcal{O}_{1}$, c'est à dire $S\left(\varphi^{\prime}\right) \in \mathcal{O}_{1}, \forall \varphi^{\prime} \in \mathcal{F}_{x}\left(\mathcal{A}_{(X, x)}\right)$. Donc $S\left(\varphi^{\prime}\right) \in$ $\mathcal{O}_{1}, \forall \varphi^{\prime} \in B_{i}(\varphi)$. Or ceci implique que $S \in \mathcal{O}_{d}$ par le lemme 1.4 p.121 de [T4]. De la même façon, soit $k=m\left(\widehat{F}_{x}^{*}(S)\right)$. Pour tout $\theta \in \mathcal{A}_{(X, x)}$, on a $: \operatorname{ord}\left({\widehat{F_{x}}}^{*}(S)(\theta)\right) \geqslant k$. Par suite, $\operatorname{Min}_{\varphi^{\prime} \in B_{\imath}(\varphi)} \operatorname{ord}\left(S\left(\varphi^{\prime}\right)\right) \geqslant k$. Mais d'après le Lemme 4.9 de la section précédente, ce minimum est inférieur ou égal à $m(S)(i+1)$. Donc $m\left({\widehat{F_{x}}}^{*}(S)\right) \leqslant \frac{1}{i+1} m(S)$. On notera donc que les constantes $a, b$ de 2) sont déterminées par le diamètre d'une boule incluse dans $\mathcal{F}_{x}\left(\mathcal{A}_{(X, x)}\right)$. La preuve de 1$) \Rightarrow 2$ ) dans 1.4 fournit un moyen effectif de déterminer un tel diamètre.

Remarque 6.3. - Les théorèmes de A.M. Gabrielov et S. Izumi, qui sont respectivement les assertions de 1) et 2) dans le cas où $(Y, y)$ est un irréductible quelconque, sont beaucoup plus profonds. L'assertion 1) dans le cadre général fût d'abord prouvée par A.M. Gabrielov. Puis S. Izumi donna une preuve de 1) notablement simplifiée dans [I4]. L'assertion 2) dans le cas général est due à S. Izumi [I1][I2][I3]. Le cas que nous traitons dans 5.2.1 a été obtenu indépendamment des façons précédentes et de manière disjointes par P.M. Eakin-J.Harris, B. Malgrange, R. Moussu-J.C. Tougeron. 
On se ramène, en général, au cas où $(X, x)$ est lisse en utilisant la résolution des singularités. Nous renvoyons le lecteur à [I4] pour une vision générale historique.

Soit maintenant $(X, x)$ un germe irréductible, $\varphi \in \mathcal{R}_{(X, x)}$ et $i \in \mathbb{N}$. Condidérons les deux assertions suivantes :

$$
\begin{array}{r}
{ }^{(1)}(T) \quad \forall S \in \widehat{\mathcal{O}}_{X, x},\left(\forall \varphi^{\prime} \in B_{X, i}(\varphi), S\left(\varphi^{\prime}\right) \in \mathcal{O}_{1} \Rightarrow S \in \mathcal{O}_{X, x}\right) \\
{ }^{(2)}(I) \exists a, b \in \mathbb{R}^{+}, \forall S \in \widehat{\mathcal{O}}_{X, x}, \operatorname{Min}_{\varphi^{\prime} \in B_{X, \imath}(\varphi)}\left(\operatorname{ord}\left(S\left(\varphi^{\prime}\right)\right)\right) \leqslant a m(S)+b .
\end{array}
$$

Les assertions $(T)$ et $(I)$ sont des conséquences très faciles de l'existence de la résolution des singularités et des théorèmes de A.M. Gabrielov et S. Izumi. Elles signifient grossièrement que le comportement d'un élément de $\widehat{\mathcal{O}}_{X, x}$ sur un ouvert de $\mathcal{R}_{(X, x)}$ décrit son comportement général. C'est pourquoi on peut considérer que de tels ouverts sont représentatifs de $\mathcal{A}_{(X, x)}$. D'autre part, une preuve directe de ces assertions $(T)$ et $(I)$ impliquerait les théorèmes de A.M. Gabrielov et S. Izumi via 1.4 comme en 5.2. Pour prouver $(T)$ et $(I)$ directement, il suffirait d'établir les propriétés 1) et 2) de 5.2 pour $\Pi:(Z, p) \rightarrow(X, x)$ l'éclatement de $(X, x)$ de centre $x$. Donc les théorèmes de A.M. Gabrielov et S. Izumi se réduisent au cas apparemment simple ci-dessus. Contrairement aux apparences, ce cas est sans doute aussi compliqué que le cas général. Cependant, une détermination «effective» de $a$ et $b$ comme dans 5.2 dans le cas de l'éclatement $\Pi:(Z, p) \rightarrow(X, x)$ de centre $x$ conduirait à des «estimations de Chevalley linéaires uniformes » comme demandées dans [B-M3] (question 1.28 p. 743).

\section{Bibliographie}

[A1] ARTIN (M.), Algebraic approximation of structures over complete local rings, Pub. Math. I.H.E.S. 36, p. 23-58 (1969).

[B] BenNetT (B.M.), On the characteristic function of a local ring, Ann. of Math. (2) 91, p. 25-87 (1970).

[B-M1] Bierstone (E.), Milman (P.D.), Relations among analytic functions I, Ann. Inst. Fourier (Grenoble), (1) 37, p. 187-239 (1987).

[B-M2] Bierstone (E.), Milman (P.D.), Uniformization of analytic spaces, Jour. A.M.S. 2, p. 801-836 (1989).

(1) (T) fût conjecturée par J.C. Tougeron dans [T4] avant qu'il n'ait connaissance du théorème de A.M. Gabrielov.

(2) $(I)$ est du à $\mathrm{S}$. Izumi [I5] utilisant la résolution des singularités et son théorème mentionné plus haut. 
Géométrie de l'espace des arcs tracés sur un espace analytique

[B-M3] Bierstone (E.), Milman (P.D.), Geometric and differential properties of subanalytic sets, Ann. of Math. 147, p. 731-785 (1998).

[D-L1] Denef (J.), Loeser (F.), Germs of arcs on singular algebraic varieties and motivic integration, Invent. Math. 135, p. 201-232 (1999).

[D-L2] Denef (J.), Loeser (F.), Geometry on arc spaces of algebraic varieties, in European Congress of Mathematics, vol I (Barcelona, 2000), Prog. Math., vol. 201, Birkhäuser, p. 327-348 (2001).

[Ga] Gabrielov (A.M.), Formal relations between analytic functions, Izv. Akad. Nauk. SSSR. 37, p. 1056-1088 (1973).

[Gr] Greenberg (M.), Rational points in henselian discrete valuation rings, Pub. Math. I.H.E.S. 31, p. 59-64 (1966).

[G-S-L-J] Gonzalez-Sprinberg (G.), Lejeune-Jalabert (M.), Sur l'espace des courbes tracées sur une singularité, Prog. Math. 134, p. 8-32 (1996).

[H1] Hickel (M.), Fonction de Artin et germes de courbes tracées sur un germe d'espace analytique, Amer. J. Math. 115, p. 1299-1334 (1993).

[H2] Hickel (M.), Calcul de la fonction d'Artin-Greenberg d'une branche plane, Pacific Journal of math. 213, p.37-47 (2004), et Preprint Université Bordeaux I No145 (Avril 2002).

[I-K] ISHII (S.) and KollÁR (J.), The Nash problem on arc families of singularities, Duke Math. J. 120, p. 601-620 (2003).

[I1] IzUmi (S.), Linear complementary inequalities for orders of germs of analytic functions, Invent. Math. 65, p. 459-471 (1982).

[I2] Izumi (S.), A measure of integrity for local analytic algebras, Pub. R.I.M.S, Kyoto Univ. 21, p. 719-735 (1985).

[I3] Izumi (S.), Gabrielov's Rang Condition is equivalent to an inequality of reduced orders, Math. Ann. 276, p. 81-89 (1986).

[I4] IzUmi (S.), The rank condition and convergence of formal functions, Duke Math. J. 59, p. 241-264 (1989).

[I5] IzUmI (S.), Note on convergence and orders of vanishing along curves, Manuscripta math. 66, p. 261-275 (1990).

[L-J] Lejeune-Jalabert (M.), Courbes tracées sur un germe d'hypersurface, Amer. J. Math. 112, p. 525-568 (1990).

[L-J-T] Lejeune-Jalabert (M.), Teissier (B.), Contribution à l'étude des singularités du point de vue du polygone de Newton, thèse université Paris VII (1973).

[Lo] Looljenga (E.), Motivic Measures, Séminaire Bourbaki 1999/2000 874, Astérisque 276, p. 267-297 (2002).

[N] NASH JR (J.F.), Arc structure of singularities, Duke Math. Journ. 81, p. 31-38 (1995).

[P] PAWLUCKI (W.), On Gabrielov's regularity condition for analytic mappings, Duke Math. J. 65, p. 299-311 (1992).

[R1] REguera (A.J.), Families of arcs on rational surface singularities, Manuscripta Math. 88, p. 321-333 (1995).

[R2] Reguera (A.J.), Image of the Nash map in terms of wedges, C.R. Acad. Sci. Paris, Ser. I 338, p. 385-390 (2004).

[S] Sebag (J.), Intégration motivique sur les schémas formels, à paraître au Bull. S.M.F. (2004).

[T1] Tougeron (J.C.), Idéaux de fonctions différentiables, Ann. Inst. Fourier 18, p. $177-240$ (1968). 


\section{Michel Hickel}

[T2] Tougeron (J.C.), Idéaux de fonctions différentiables, Ergeb. der Mathematik 71, Springer Verlag (1972).

[T3] Tougeron (J.C.), An extension of Whithney's spectral theorem, Pub. Math. I.H.E.S. 40, p. 139-148 (1971).

[T4] Tougeron (J.C.), Courbes analytiques sur un germe d'espace analytique et applications, Ann. Inst. Fourier 26, p. 117-131 (1976). 